\title{
THE EFFECTS OF LAND VERSUS AQUATIC PLYOMETRICS ON POWER, TORQUE, VELOCITY, AND MUSCLE SORENESS
}

\author{
A Thesis \\ Presented in Partial Fulfillment of the requirements for \\ the Degree of Master of Arts in the \\ Graduate School of The Ohio State University
}

By

Leah Elizabeth Robinson, B.S.

$* * * * *$

The Ohio State University

2002

Master's Examination Committee:

Approved by

Janet Buckworth, Ph.D., Advisor

Steven T. Devor, Ph.D.

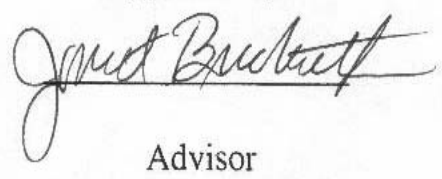

Mark A. Merrick, Ph.D.

College of Education

School of PAES 



\begin{abstract}
Researchers have previously examined only the effects of land plyometrics on power, force, and velocity. The purpose of this study was to compare changes in performance outcome and muscle soreness between plyometric training on land and plyometric training conducted in an aquatic setting. Thirty-two physically active collegeaged female students with varied sport experience and no previous orthopedic or musculoskeletal injuries to the lower extremities that exhibit chronic problems were matched on vertical jump and randomly assigned to an 8-week land plyometric or an 8week aquatic plyometric program. Performance outcome variables were peak power [vertical jump distance in cm using a vertical (squat jump) jump test], peak torque [torque in $\mathrm{N} \cdot \mathrm{m}$ using BIODEX isokinetic testing] and velocity $\left[\mathrm{m} \cdot \mathrm{s}^{-1}\right.$ in a 40 -meter sprint $]$ and were assessed pre-training, mid-training, and post-training. Muscle soreness of the rectus femoris, biceps femoris, and gastrocnemius muscle was estimated using an established ordinal scale and self-report of pain sensitivity upon muscle palpation as measured with an algometer (Algometer FDK40, Scrip Inc.; Peoria, IL). Muscle soreness and pain sensitivity were assessed at baseline (first week of training) and when training intensity was increased at week 3 and week 6 at 0, 48, and $96 \mathrm{hr}$ post-training bout. The exercise
\end{abstract}


protocols involved three exercise sessions per week lasting 65 minutes, including a 15 minute warm-up. The training protocols were identical except that land plyometrics was conducted on a gymnasium floor and the aquatic plyometrics was conducted in 4 to $4 \frac{1 / 2}{12}$ feet of water.

Multivariate and univariate analysis of variance set at a statistical significance of $p \leq 0.05$ were used for statistical analyses. Performance outcome results indicated that there was a main effect for time. Post-hoc tests revealed that pre-training $<$ mid-training $<$ post-training, $p \leq 0.001$, for power output, torque, and velocity. There was no main effect for treatment and no treatment by time interaction effect indicating that there was no performance outcome difference between the two treatment groups. The perception of muscle soreness was significantly greater, $p=0.01$, in the land plyometric training group than the aquatic plyometric training group at baseline and when the training intensity was increased at week 3 and week 6 at $48 \mathrm{hr}$ and $96 \mathrm{hr}$ post-training bout. Therefore, it was concluded that aquatic plyometrics provides the same performance enhancement benefits when compared to land plyometrics with a reduced amount of muscle soreness. 
This thesis is dedicated to my mother and father.

Thanks for believing in me. 


\section{ACKNOWLEDGEMENTS}

I wish to thank my advisor, Janet Buckworth, Ph.D., for all of her guidance, time, and support in helping me complete my Master's of Arts Program. I am also grateful for her patience in reading and correcting this project.

I wish to that Mark A. Merrick, Ph.D. and Steven T. Devor, Ph.D. for their

countless time and support. All of your assistance made this thesis project possible.

I wish to thank all of my subjects for their time, dedication, and effort. Without them, this thesis would have been impossible.

I am indebted to Brandy Mabra, Andrea Malyk, Aaron Robinson, Luke Schefft, and Chad Scott for assisting me with training and testing sessions. I could not have completed this project without you.

Finally, I wish to thank Dominic for his wisdom, time, and belief in my abilities while pushing me to excel. 
VITA

October 18, 1978

Born - Fayetteville, North Carolina

2000

B.S., Physical Education (Biology minor),

North Carolina Central University

$2000-2002$

Graduate Associate,

The Ohio State University

\section{PUBLICATIONS}

\section{Research Publication}

L.D. Wilkin, J.M. Lekan, L.E. Robinson, AND S.T. Devor. Assessment of degree of injury following a skeletal muscle contusion. Med. Sci. Sports Exerc. 34(5):S184, 2002.

\section{FIELD OF STUDY}

Major Field: Education

Specialization: Sport and Exercise Science 


\section{TABLE OF CONTENTS}

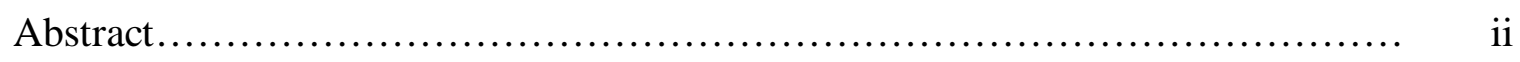

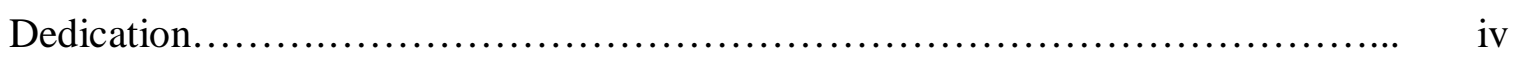

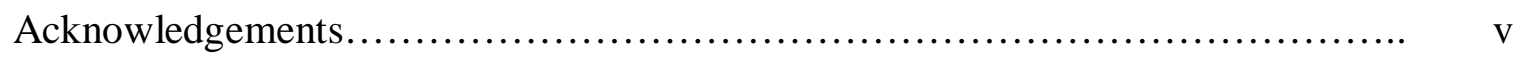

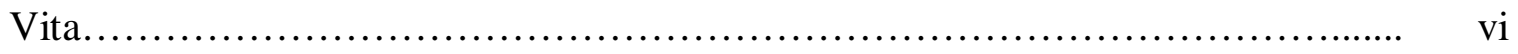

List of Tables................................................................... xi

List of Figures......................................................... xii

1. Introduction................................................................... 1

Purpose of this Study ......................................... 3

Research Hypotheses........................................ 4

Definitions....................................................... 5

Limitations................................................ 9

2. Review of the Literature.............................................. 10

History of Plyometrics............................................. 11

Plyometrics in Sport Training Programs......................... 13

Plyometric Training Effects on Power and Force Adaptations....... 15

Plyometric Training Effects on Velocity Adaptations.............. 19

Major Drawbacks of Plyometric Training....................... 20 
Potential Injuries from Plyometric Training.................... 21

Delayed Onset Muscle Soreness.............................. 22

Markers for Muscle Damage................................ 22

Aquatic Training........................................... 24

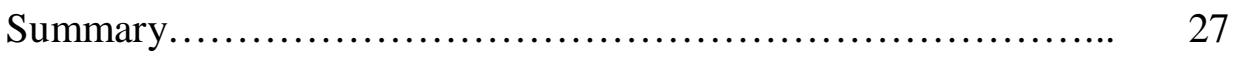

3. Methods............................................................. 28

Subjects................................................. 28

Procedure................................................... 30

Plyometric Training Protocol............................... 32

Measurements.......................................... 33

Peak Power Output.................................. 33

Peak Torque Production............................. 34

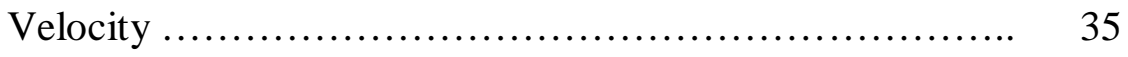

Muscle Soreness....................................... 36

Pain Sensitivity................................... 36

Body Composition Assessment...................... 38

Anthropometric Assessments......................... 39

Statistical Analysis.................................... 39

4. Results............................................................. 41

Performance Outcomes................................... 43

Muscle Soreness.......................................... 48

Muscle Soreness Scale.............................. 48 


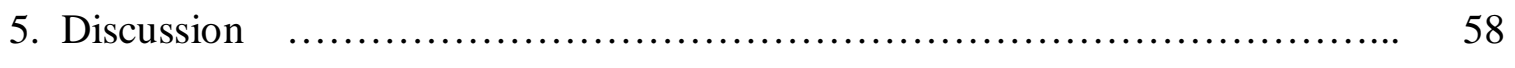

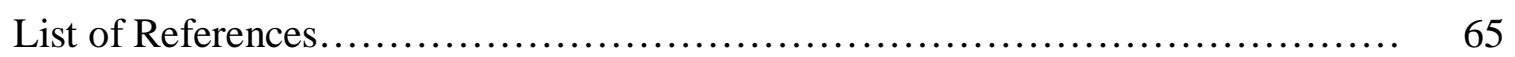

Appendices........................................................... 75

APPENDIX A Institutional Review Board Approval Form........ 75

APPENDIX B Informed Consent.......................... 78

APPENDIX C Paffenbarger Physical Activity Questionnaire..... 82

APPENDIX D Demographic Form........................... 85

APPENDIX E General Medical History Form................... 88

APPENDIX F Musculoskeletal Medical History Form........... 91

APPENDIX G Plyometric Training Study Timeline.............. 95

APPENDIX H Warm-Up (Stretching) Program................. 97

APPENDIX I Plyometric Training Protocol................... 101

APPENDIX J Peak Power Output Protocol.................... 110

APPENDIX K Peak Torque Production Protocol................ 112

APPENDIX L Velocity - 40 meter Sprint Protocol.............. 115

APPENDIX M Muscle Soreness Scale/Protocol................. 117

APPENDIX N Pain Sensitivity Algometer Protocol.............. 121

APPENDIX O Body Composition Assessment Protocol.......... 124

APPENDIX P Anthropometrical Measurement Protocol.......... 126

APPENDIX Q Rectus Femoris Muscle Soreness................ 128

APPENDIX $\quad \mathrm{R} \quad$ Biceps Femoris Muscle Soreness................ 130 
APPENDIX $\quad \mathrm{S}$ Gastrocnemius Muscle Soreness................. 132

APPENDIX $\quad \mathrm{T} \quad$ Rectus Femoris Pain Sensitivity.................. 134

APPENDIX U Biceps Femoris Pain Sensitivity................ 136

APPENDIX V Gastrocnemius Pain Sensitivity................. 138

APPENDIX W Data Collection Sheet......................... 140 


\section{LIST OF TABLES}

$\underline{\text { Table }}$

$\underline{\text { Page }}$

3.1 Demographic characteristics for $n=32$ pre-training ..................... 29

4.1 Descriptive statistics for plyometric treatment groups at pre-training....... 42

4.2 Effects of plyometric training on peak power output..................... 44

4.3 Effects of plyometric training on peak torque production miomietrically... 45

4.4 Effects of plyometric training on peak torque production pliometrically.... 46

4.5 Effects of plyometric training on velocity......................... 47

A.1 Muscle soreness perception of the rectus femoris muscle................ 129

A.2 Muscle soreness perception of the biceps femoris muscle................ 131

A.3 Muscle soreness perception of the gastrocnemius muscle............... 133

A.4 Pain sensitivity of the rectus femoris muscle.......................... 135

A.5 Pain sensitivity of the biceps femoris muscle....................... 137

A.6 Pain sensitivity of the gastrocnemius muscle......................... 139 


\section{LIST OF FIGURES}

$\underline{\text { Figure }}$

4.1 Differences in muscle soreness between land and aquatic plyometric training groups: rectus femoris.

4.2 Differences in muscle soreness between land and aquatic plyometric training groups: biceps femoris

4.3 Differences in muscle soreness between land and aquatic plyometric training groups: gastrocnemius

4.4 Differences in pain sensitivity between land and aquatic plyometric training groups: rectus femoris.

4.5 Differences in pain sensitivity between land and aquatic plyometric training groups: biceps femoris

4.6 Differences in pain sensitivity between land and aquatic plyometric training groups: gastrocnemius. 


\section{CHAPTER 1}

\section{INTRODUCTION}

Three decades have passed since Title IX (Educational Amendment Act of 1972: U.S.C. 1681-1688 Title 20 Chapter 38 - Discrimination Based on Sex or Blindness) was enacted, which mandated that high schools and colleges not discriminate based on gender in any educational programs or activities that receive federal funding (57). Since this law was enacted in 1972, there has been a continuing increase in the number of females participating in sports. Female sports were once dominated by a slow and defensive style, but now they are played with power, speed, and precision. This change in playing styles requires an innovative conditioning program such as plyometrics that enhances agility and strength. Plyometric training provides the link between velocity and force to

produce power, and enables muscles to reach their maximum force in the shortest amount of time (17).

The change in female playing styles has lead to a corresponding increase in injuries. Female athletes tend to have a greater injury rate than male athletes. Data have established that females participating in sports sustain Anterior Cruciate Ligament (ACL) injuries 2 to 8 times more than their male counterparts (42). It has been suggested that 
this epidemic may be due to the female's greater joint laxity and range of motion, as well as hormonal, anatomical, muscular, and neuromuscular differences (87).

Currently, attention is being drawn toward plyometric training as a method for injury prevention in female athletes. Plyometric training has been shown to enhance neuromuscular activity (11). The potential improvement in neuromuscular activity was the basis for a study by Hewett, Riccobene, and Lindenfeld testing the effects of a sixweek preseason plyometric training program on the incidence of non-contact ACL injuries in 800 high school female athletes (34). During the subsequent competitive season, the female athletes who did not participate in the plyometric training had twice as many ACL injuries (34).

The research consensus is that plyometric training can enhance an individual's athletic performance, and the use of plyometrics as a training modality to reduce injuries in females and to enhance performance has increased. However, there could be a major drawback involved with plyometric exercises, specifically, an increased risk of injuries caused by external forces acting upon a joint that momentarily surpass the structural integrity of the muscles, bones, and connective tissue (13). Could we be making athletes more susceptible to another form of injury while decreasing the risk of ACL injuries and boosting performance? Horrigan and Shaw (41) cited several examples of elite athletes who performed plyometric exercises that resulted in serious injuries (i.e., tendonitis, muscle strains, and jumper's knee), which caused them to miss practices and sporting events. Researchers, strength coaches, athletic and personal trainers need a form of training that will not only enhance performance, but also reduce the likelihood of other training injuries. 
Aquatic training offers efficiency, comfort, safety, and training at any level of intensity. Performing exercises in the vertical plane (axis) maximizes resistance and increases turbulence and drag, which helps to strengthen the active muscle. It can also be expected that injury rate would be lower in water due to the buoyancy that water provides. However, researchers have not examined the differences in muscle injury and muscle soreness occurrence between plyometric training on land and in water. If aquatic plyometric training can provide comparable training gains with reduced risk of injury, coaches and strength specialists would have a more viable training option for conditioning that would enhance performance while reducing the risk of injury.

\section{PURPOSE OF THIS STUDY}

The controversy over the safety of plyometrics and the lack of research concerning the effects of plyometrics training in aquatics lead to the design of this study. The purpose of this study was to determine the effectiveness of land versus aquatic plyometrics on power, torque, velocity, and muscle soreness in females. The effects of the training environment (land versus aquatic) on performance outcome and muscle soreness during and after plyometric training were addressed. The objectives of the study were (a) to compare changes in power, torque, and velocity between land and aquatic plyometric training programs and (b) to compare degree of muscle soreness overtime between land and aquatic plyometric training groups. 


\section{RESEARCH HYPOTHESES}

1. After 8-weeks of training, aquatic plyometric training would result in no significant difference in peak power output as measured by the Sergeant vertical jump test when compared with land plyometric training.

2. After 8-weeks of training, aquatic plyometric training would result in no significant difference in peak torque production as measured by isokinetic testing when compared with land plyometric training.

3. After 8-weeks of training, aquatic plyometric training would result in no significant difference in velocity when compared with land plyometric training.

4. Aquatic plyometric training would result in a significantly lower level of muscle soreness as measured through self-report and pain sensitivity measured with an algometer when compared with land plyometric training during training week-1, week-3, and week-6.

\section{SIGNIFICANCE OF THE STUDY}

This study provided information about muscle soreness and muscle injury

associated with land plyometrics over 8-weeks of training. It also examined the effects of a "new" form of a plyometric intervention (i.e., aquatics) that may enhance physiological adaptations as well as reduce muscle soreness and muscle injury. 


\title{
DEFINITIONS
}

Aerobic exercise: A mode of exercise that requires the use of oxygen; uses adenosine triphosphate, triglycerides, and aerobic glycolysis for primary energy sources.

\begin{abstract}
Algometer (pressure threshold meter): An instrument used for the evaluation of the minimum pressure that induces pain or discomfort.
\end{abstract}

Anaerobic exercise: A mode of exercise that does not require the use of oxygen; uses the phosphagen system and anaerobic glycolysis for primary energy sources.

Aquatic fitness/exercise program: Exercises performed primarily in a vertical plane in shallow or deep water; uses movements that amplify drag by unstreamlining the body to create resistance.

Buoyancy: The upward force acting in the opposite direction of gravity during immersion.

Exercise: A subclass of physical activity, defined as planned, structured, and repetitive bodily movement done to improve or maintain one or more components of physical fitness. 
Force: Any force that causes a body to move or accelerate; a vector quantity that tends to produce a change in the motion of objects.

Healthy: Individuals who do not currently have any infectious disease, are not taking any prescription medications that may effect performance, and have no history of any cardiopulmonary diseases or lower extremity orthopedic/musculoskeletal injuries that exhibit chronic problems.

Intensity progression: A method of increasing the workload by gradually changing the degree or level of physical difficulty.

Miometric contraction: Usually referred to as concentric contraction; it describes the shortening of an active muscle.

Muscular strength: The ability of a muscle to exert force; usually measured as the maximum load that can be lifted at once.

Physical activity: Bodily movements that are produced by the contraction of skeletal muscle and that substantially increase energy expenditure.

Physically active: Subjects who self-report performing moderate to high intensity physical activity for at least 30 minutes a day 3 times a week for the past 6 months. 
Pliometric contraction: Usually referred as to eccentric contraction; it describes the lengthening of an active muscle.

Plyometrics: Type of exercise that encompasses the stretch-shortening cycle; an activated muscle stretches or lengthens (pliometric) and immediately performs a shortening (miometric) contraction.

Power: A scalar quantity that measures the rate at which work is done.

Properties of water: The unique characteristic of water that effects the body during exercise including buoyancy and wave drag resistance.

Sedentary: Is not participating in a regular exercise program for at least 6-months prior to joining this study.

Sport experience: Includes the participation in organized sport and recreation activities; either scholastic, collegiate, or college club and/or intramural for at least 3 years.

Torque: Any influence that causes a body to move or accelerate; a vector quantity that tends to produce a change in the motion of objects.

Velocity: The rate at which a body moves in a given directions; a vector quantity whose magnitude is expressed units of distance and time. 
Wave drag resistance: The force acting on a body in motion through a fluid in the direction opposite to the body's motion.

\section{DELIMITATIONS OF THE STUDY}

The study was delimited to the following conditions.

1. All assessments and training sessions were conducted by trained and experienced technicians.

2. The same training and testing facilities, research assistants/technicians, and method, were used for each assessment and session.

3. Each training session was held for 65 minutes, three days per week at the same time each day.

4. Water temperature remained at a constant temperature of $77^{\circ} \mathrm{F}\left(25^{\circ} \mathrm{C}\right)$ to $79^{\circ} \mathrm{F}\left(26^{\circ} \mathrm{C}\right)$ throughout the eight weeks.

5. All subjects were healthy, physically active individuals with some sport experience. 


\section{LIMITATIONS OF THE STUDY}

This study was limited by the following factors.

1. The subjects were volunteers that were self-selected.

2. The subjects that participated in the study were all physically active college-age Caucasian females who were currently or previously involved in sports and had greater than a high school education. This group may not be representative of the general population, but more representative of a collegiate female athlete.

3. The duration of the training protocol was 8 -weeks. Most strength training studies are 10 - 12 weeks in duration. However, 8-weeks is usually the duration of pre-season athletic strength and conditioning programs.

4. Some variables could not be well controlled. Factors such as diet, motivation, and the level of cooperation of the subjects during the training and pre-/mid-/post-training testing sessions may have had an impact on the results.

5. Self-report muscle soreness was assessed through the subjects' perception. A more precise measure would include a biochemical analysis to evaluate chemical markers for muscle soreness and muscle damage. 


\section{CHAPTER 2}

\section{REVIEW OF THE RELATED LITERATURE}

\section{INTRODUCTION}

Several bodies of literature were reviewed in the process of designing and conducting this research study. The following sections include an overview of the importance of plyometrics in training programs as a method to enhance power output, force production, and velocity. The first section will provide the history of plyometric training and the importance of incorporating plyometrics into training programs. The next section will include a review of plyometric training effects on skeletal muscle plasticity. Studies reporting the effects of muscle injuries and muscle soreness during and following plyometric training were also included in the literature review. Lastly, this chapter will conclude with a review of the benefits and effects of aquatic training. 


\section{HISTORY OF PLYOMETRICS}

Plyometric training is a form of explosive, physical training that was first recognized during the 1960s Summer Olympic Games when Russia began to produce superior athletes in track and field, gymnastics, and weightlifting (17). The most prominent Russian coach, Yuri Veroshanski, accredited Russia's athletic success to the incorporation of jump-training into their training programs (68). Veroshanski proclaimed that their experiments with jump-training enhanced their athletes' reactive ability and neuromuscular activity (68). This jump-training phenomenon was later coined as plyometrics, which was derived from the Greek term "pleythyein" meaning "more measure" (68). Since the 1960s, American athletes of all levels have used plyometrics as a training modality to produce a positive effect on the muscle's physiological characteristics and athletic performance.

The neuromuscular mechanism of the plyometric action is a myotatic (stretch) reflex (17). This reflex is also commonly referred to as the stretch-shortening cycle. Plyometric activities are created by an intense pliometric contraction of a muscle followed immediately by a rapid miometric contraction; this action produces a forceful, explosive movement $(1,8,17)$. Plyometrics evokes the elastic properties of the muscle fiber and connective tissue, which allow the muscle to store energy during the deceleration phase and release that energy during the acceleration phase $(4,16)$. This results in muscles being trained under tensions greater than those achieved by conventional slow-speed resistance training (39). 
The stretch reflex is one of the fastest reflexes in the body due to its direct connection between receptors in the muscle and the cells in the spinal cord (17). Plyometric training does not enhance the time of the stretch reflex action; however it changes the strength of the reflex's muscle contraction (17). The key concept to know is that a faster stretched muscle results in greater force development. Lundin and Berg (53) described the process of plyometrics as:

A muscle forcibly stretched before a contraction uses the stretch reflex to activate the muscle to shorten vigorously, and the elastic nature of the muscle fibers allows the muscle to store energy during negative work, that will be released during the shortening contraction. (p. 25)

Thomas also agreed with the concept of the stretch-shortening cycle by stating that "muscles that contract immediately after being stretched produce greater force than a muscle, which contracts without pre-stretch" $(81$, p. 49). The quickness in the ability to convert from a pliometric contraction to a miometric contraction is the key to exploiting the muscle's elastic compositions (53). Therefore, it is implied that plyometric training improves the velocity of the stretch-shortening cycle (17). The potential for the muscle to store elastic energy is due to the muscle's series elastic components (tendons and crossbridge characteristics of actin and myosin) and sensors in the muscle spindle that play a role in presetting the muscle tension (17). The storage of elastic energy caused by plyometrics enables the muscles to generate greater force and power. For this reason, plyometric training plays an essential role in strength and conditioning programs. 


\section{PLYOMETRICS IN SPORT TRAINING PROGRAMS}

It is essential to design training and conditioning programs to be as specific as possible to the actual performance and environment of the activity. Plyometric training is a novel form of conditioning and relates to the specificity principle.

Fox, Bowers, and Foss (28) stated:

Experience has taught successful coaches that in order to increase the performance of their athletes; a specific training program must be planned for each athlete. In other words, the training program must be relevant to the demands of the event for which the athlete is being trained. (p.171)

Conditioning activities during in-season training must be specific to the skills needed for the activity being performed. The Specific Adaptation to Imposed Demands (SAID) principle states that conditioning should consist of both metabolic and neuromuscular specificity (3). This implies that the training procedures must use the identical cardio-respiratory and energy pathways, along with the same movement patterns/joint angles and specific muscles used in the activity.

Plyometric training has been described as exercises or drills that combine speed and strength to produce an explosive movement and an increase in power (17).

Plyometric training also focuses on the specific demands of athletic events, especially on movement patterns and muscle utilization. Plyometrics have been promoted as being specific to almost every sport due to the combination of force and velocity development (90). Thus, there are clear benefits for strength specialists and coaches who incorporate plyometrics into their training programs. 
Radcliffe and Farentinos (68) reviewed plyometric training in the sport performance literature in the 1980s. They described the four basic plyometric drills common in training programs. These basic plyometric drills (i.e., jumps, hops, bounds, and shock drills) will induce the stretch-shortening cycle in order to enhance explosive movements (68). Jumps are defined as movements ending with a two-foot landing. Examples included jumping in place or the standing jump. Hops are classified as a movement beginning and ending with a one- or two-foot landing of the same foot or feet. Bounds are classified as a series of movements in a horizontal direction where the individual lands successively on both or alternate feet. Bounds can be classified as long or short response. Finally, shock drills include box jumps (depth jumps) when individuals jump on and off of boxes of varying heights.

An effective plyometric program achieves its outcome through the manipulation of four variables: volume, intensity, frequency, and recovery (68). Volume relates to the total work performed for each session. Intensity refers to the difficulty and the number of exercises performed in each session. Frequency refers to the number of repetitions that an exercise is performed, and the number of times a session takes place per cycle (week). Recovery is the rest between each set and is a key component in plyometric training. An ideal recovery rate should be a 1:5 to 1:10 ratio (17). For example, if an exercise takes 10 seconds to complete, there should be a 50-100 second rest (17). 


\section{PLYOMETRIC TRAINING EFFECTS ON POWER AND FORCE ADAPTATIONS}

Explosive leg power constitutes a crucial component in numerous athletic events. Vertical jump height demonstrates a positive correlation with lower body power (66). Jumping ability is critical in the execution of many athletic skills, such as high jumping, rebounding and blocking in basketball, and spiking in volleyball. The neural coordination of the movement (50) and the rate of force development by the ankle, knee, and hip joints and muscles (24) are major determinants of vertical jump performance.

Previous studies have examined vertical jump and power changes in a variety of strength training programs. Recently, Potteiger et al. (66) examined changes in muscle power output and fiber characteristics following an 8-week plyometric training program in 19 physically active college-aged males. The training regiment was three times a week and consisted of a plyometric exercise program and a combination program of plyometrics and aerobics. The plyometric training protocol consisted of vertical jumps, bounds, and depth jumps. The combination program included the identical plyometric protocol and was immediately followed by a 20 -minute aerobic workout at $70 \%$ of their maximum heart rate. The results showed a significant improvement in peak power and an increase in type I and type II fiber cross-sectional area $\left(\mathrm{CSA}_{\mathrm{f}}\right)$ from pre-training to post-training within all of the groups.

Two similar experiments were performed to examine the effects of resistance training and depth jump training on vertical jump and lower extremity force production (21). The first experiment evaluated the outcome of a two times a week combination of jumping program and resistance training on vertical jump and force produced in the 
lower extremities in 12 college-aged males for 4-weeks (21). Four subjects were placed in either a (1) maximum vertical jumps, (2) 0.3 meter depth jumps, (3) a combination of 0.75 meters and 1.10 meters depth jumps. Resistance training followed each of the jump programs. The three training program resulted in improvements in vertical jump and force produced when squatting and performing an isometric knee extension. However, no significant differences existed between the three jumping programs. The second experiment compared the effects of a 16-week combination of resistance training and depth jump training program with a resistance training program in 16 male collegiate volleyball players (21). The training occurred two times a week and the depth jump height was set at $0.75 \mathrm{~m}$ and $1.10 \mathrm{~m}$. The depth jump group made significant gains in vertical jump and force produced by the lower extremities; however, the weightlifters only saw improvements in force development. The conclusion from these two experiments was that jump-training alone and a combination of resistance training and jump-training are effective in improving vertical jump performance, power output, and force production.

Other studies have examined the effects of plyometrics on lower extremity power production. For instance, Anderst et al. (2) examined the effects of a three day a week 10-week plyometrics and explosive resistance training (weighted squat jumps) on drop jumps and countermovement jumps in 12 college-aged males. The training resulted in significant improvements in takeoff velocity, takeoff power, and jump height. Results also showed that there were no differences between the plyometrics and explosive resistance training group. Wilson (86) conducted a study to evaluate the effects of a three day a week, 10-week traditional resistance training (heavy squats), plyometric training, 
and explosive resistance training (weighted squat jumps) at the load that maximized power output on 64 untrained male subjects. The results indicated that the groups who performed explosive resistance and plyometric training had a significant improvement in power output compared to traditional resistance training.

It is also possible to perform upper-body plyometrics to improve the power production of the upper extremity muscles. Crowder (22) examined upper-body power production in 34 male subjects by incorporating the use of upper-body plyometrics. The subjects were assigned to three days a week of either push-ups or hand-clap push-ups (plyometric) training, with resistance training followed each push-up training session. After the 10-week training programs, the hand-clap push-ups (plyometric) group outperformed the traditional (regular push-ups) group on upper body power production.

An earlier investigation performed by Parcells (63) evaluated the effects of a 6week plyometric depth jumps and resistance training program on vertical jump performance. Forty-five college-aged males participated in the training programs twice a week. The resistance training group performed 3 sets of 8 repetitions of half-squat heel raises with $50 \mathrm{lbs}$, with a two repetitions increase each week. The plyometric training group performed depth jumps with a progression in height from $0.8 \mathrm{~m}$ to $1.1 \mathrm{~m}$ through the training program. The results indicated that only the plyometric depth jump group significantly improved on vertical jump performance. The results of these studies are consistent with the finding of other studies that show that the end results of plyometric training are improvements in lower body power production $(7,10,66,86)$, upper body power output (22), and vertical jump performance (1, 2, 21, 63). 
Plyometric training also results in significant improvements in the maximal rate of force development (32). Hakkinen et al. (32) examined the effects of heavy resistance training versus plyometric training on lower extremity electromyographic (EMG) activity. Ten (10) physically active male subjects participated in a progressive resistance and plyometric training program three times a week for 24-weeks. The training program was followed by a 12-weeks detraining period. The results clearly illustrate the potential of plyometric exercises to enhance the rate of EMG and tension development during isometric force-time analysis and depth jump performance. Heavy resistance training increased max EMG by $0.3 \%$ and force by $11 \%$, but it had little if any effect on EMG (0 $\%)$, tension development $(0.4 \%)$, or drop jumps performance (0\%). Conversely, plyometric training increased max EMG by $8 \%$ and force by $27 \%$, and it had a greater effect on EMG (38\%), tension development (24\%), and drop jump performance (19\%).

Although improvements in the rate of force development have been demonstrated (e.g., 32), few studies have explored the outcome of plyometrics on force production. Fatourus et al. (24) examined the effects of a 12-week plyometric training, resistance training, and a combination of the two programs on force production, mechanical power, and vertical jump performance in 41 college-aged males. Training sessions occurred three times a week. Results showed that all training treatments elicited improvements in all the tested variables. However, the combination training group produced significantly greater improvements in all the variables when compared with the plyometric training and resistance training alone. A study performed by Clutch et al. (21) mentioned earlier in this review also showed that a combination of plyometrics and resistance training would improve force production. These studies demonstrated that plyometric drills alone 
and incorporated with traditional and Olympic-style resistance training had an effective outcome on force production, explosive power, and jumping ability $(21,24)$. In addition, it has been hypothesized that plyometrics will have the same positive training effect on an individual's ability to produce speed.

\section{PLYOMETRIC TRAINING EFFECTS ON VELOCITY ADAPTATIONS}

Track athletes and coaches constantly incorporate plyometrics into their daily training as a modality to improve speed and sprint performance (90). It is generally accepted that the more specific a training exercise mimics the competitive movement, the greater the transfer of the training effect to the competition $(23,73)$. Sprinters incorporate bounding in training programs because they require power and force for movements in the horizontal plane (83). The contact phase is the first stage in sprinting (5). During this phase, the sprinter begins with a pliometric contraction of the leg extensor muscle as the center of gravity of the body is lowered slightly (5). The pliometric contraction is immediately followed by the push-off or propulsive phase when a miometric contraction occurs with the same muscle (5). This muscle contraction sequence occurs with every foot contact, and it is described as the stretch-shortening cycle. According to Schmidtbleicher (75), the stretch-shortening cycle found in elite athletes varies from long (> $350 \mathrm{~ms})$ to short $(<250 \mathrm{~ms})$. The quickness of the stretchshortening cycle is the key to propulsion in sprinting (5). 
The purpose of a study by Rimmer \& Sleivert (69) was to determine the effects of an 8-week sprint-specific plyometric intervention program, sprint-training intervention program, and a control program on sprint performance in 26 college-aged males. A total of 15 training sessions was conducted. The results showed that sprint time over $40 \mathrm{~m}$ was significantly improved in the plyometric group; these changes were significantly different from the changes seen in the control group. The changes observed in the sprintspecific plyometric group were not different in the magnitude of the improvement when compared with the gains in the sprint-training group. The plyometric intervention also caused improvements over the interval within the $0-40 \mathrm{~m}$ distance. There was no change in stride length or stride frequency, however ground contact time decreased by $4.4 \%$ in the plyometric-trained subjects. This study concluded that a sprint-specific plyometric program could also serve as a training approach to improve sprint performance time.

\section{MAJOR DRAWBACKS OF PLYOMETRIC TRAINING}

Many activities in sports and conditioning programs (i.e., plyometric training) require landing from a jump, which leads to impact forces acting on the body that increase the risk of injury. Impact forces (ground reaction forces) are the forces produced by the collision of two objects, which causes a shock wave to be generated along the body (61). Ground impact forces can reach maximal force within $50 \mathrm{~ms}$ after first contact (61). Impact forces at foot-strike range from 2 to 5 times an individual's body weight and can vary between 7 to 12 times the body weight when performing activities 
(67). For example, ground impact forces for elite triple jumpers and gymnasts can range from 8.8 to 14.4 times the body weight (67) or from 572 to $936 \mathrm{~kg}$ for a $65 \mathrm{~kg}$ person. The forces generated from ground contact can make coaches and strength specialists apprehensive about including plyometrics in strength and conditioning programs.

\section{POTENTIAL INJURIES FROM PLYOMETRIC TRAINING}

Plyometric exercises are associated with fear of injury, muscle damage, and muscle soreness due to intense pliometric contraction and ground impact forces. A majority of lower back injuries, meniscal damage, patellar tendonitis, Achilles tendon strains, heel bruises, plantar fascia strains, vertebral compression fractures, and synovial lining inflammation have been attributed to the repetitive ballistic movements of plyometrics (13). Each individual muscle fiber can exert a larger force while being stretched than it can while being shortened; thus, fewer fibers are recruited to exert a given amount of force (46). However, the larger forces per muscle fiber developed during the pliometric contraction result in greater muscle damage (76). Pliometric exercises have been shown to produce greater amounts of muscle soreness and larger elevations of plasma creatine kinase when compared to miometric exercises (20). It can be concluded that the pliometric phase of the plyometric exercises produces more microscopic damage to the muscle fiber, hence propagating a higher degree of muscle soreness compared to the miometric phase. 


\section{DELAYED ONSET MUSCLE SORENESS}

Delayed onset muscle soreness (DOMS) is a normal response to unusual exertion, and is part of an adaptation process that leads to greater stamina and strength as the muscle recovers and builds (33). DOMS appears hours after the exercise is over. The soreness is generally at its worst within the first two days following the activity and subsides over the next few days. DOMS is thought to be a result of minute tearing of the muscle fibers (44). The amount of tearing and soreness depends on the intensity, duration, and type of exercises being performed (44). It appears that pliometric contractions are responsible for muscle soreness and contraction-induced muscle damage (36). In fact, pliometric contractions can result in severe muscle soreness upon palpation (74), temporary loss of the exercised muscle's range of motion $(18,74)$, intramuscular swelling and edema $(18,31)$, and a decline in force production (18).

\section{MARKERS FOR MUSCLE DAMAGE}

The proposed chemical agents of DOMS include the protein enzyme products of muscle breakdown, such as creatine kinase, inorganic phosphate, and metabolic waste products such as lactic acid (60). Elevated pressure and mechanical distortion of the tissue, which accompanies edema, could also activate nociceptors in the muscle and elicit the sensations of DOMS. The amount of muscle damage depends on the mode of exercise and the exercise intensity (44). 
It has been reported that delayed pain was closely associated with tension within the muscle, which suggests that soreness had its origin in some sort of rupture occurring within the muscle itself (40). If fact, evidence indicates that skeletal muscle damage may be the primary mechanism contributing to muscle soreness (40). Friden et al. (29) were the first to demonstrate changes in skeletal muscle fibers following an exercise protocol of repeated stair descents, which resulted in severe DOMS. The post-exercise results demonstrated myofibrillar disturbances, causing Z-band disruption and streaming.

Jamurtas et al. (43) compared the effects of a bout of plyometric exercises on muscle soreness and plasma creatine kinase levels with pliometric and miometric exercises on twenty-four untrained and inactive college-aged males. The subjects performed two exercise bouts separated by 6-weeks. The results indicated that the plyometric and pliometric exercise groups had a significantly higher perception of DOMS when compared with the miometric exercise group. The elevated perception of muscle soreness was probably due to the lengthening contraction causing damage to the muscle fiber or musculotendonious junction. Due to the subjects being untrained and inactive, no significant difference was present when examining plasma creatine kinase levels. The level of inactivity in the subjects can explain the results in muscle damage because any type of unaccustomed physical activity or exercise will cause injury to an inactive and untrained individual. 


\section{AQUATIC TRAINING}

Aquatics refers to a term for various disciplines of water activities that have increased in popularity within the past decade. In 2000, there was an estimated 9 million water exercisers in the United States with a $50 \%$ increase in the number of water aerobics classes from 1995 to 2000 (77). The recent growth in aquatic activities is due in part to the properties of the water (i.e., resistance and buoyancy).

Water creates a "non-impact medium" that produces little strain on muscle, bones, and connective tissue when compared with land activities (65). Water also provides buoyancy that reduces weight-bearing stress, however, movement in water increases the resistance $(71,78)$. An increase in resistance results in a greater workload. Weightbearing activities on land places stress on the lower limbs, and this stress is considerably reduced in water due to its buoyancy $(30,71)$. Although water reduces the effects of weight-bearing on the skeletal joints while at rest, the resistive effect of water on joint movements provides exercise loading, enhancing muscular tension beyond that achieved on land (30).

Aquatic training also allow individuals to exercise almost every muscle and joint in the body at the same time, while conducting heat away from the body more efficiently than air $(15,71)$. Aquatics also provide resistance in all directions and 12 times the resistance when compared to land activities (65). For these reasons, exercising in water may be more appropriate than land exercises for individuals who are obese, ailed with arthritis and/or musculoskeletal injuries, pregnant, or part of the older population. 
For example, Weinstein (84) examined and discussed the benefits of two aquatic programs for older adults. An 8-week Senior Splash aerobic swim class that included exercises in the water, exercises in the water against the pool wall, and dance routines was compared to the Arthritis Aquatic Program, which conformed to the guidelines set by the Arthritis Foundation. The Arthritis Aquatic Program was much slower than the aquatic aerobic class, and the subjects in the Arthritis Aquatic Program had various forms and sites of arthritis. Subjects in both programs received physiological and psychological benefits. These benefits included an increase in strength and joint range of motion, along with a boost in social interaction and decreases in depression and isolation. The results of this study agree with the perspective that aquatic programs have significant benefits for the elderly population suffering with arthritis $(70,79)$.

Binkley (9) examined the effects of a 10-week water exercise program on muscular strength in 10 elderly women. The water exercise programs occurred three times a week. Muscular strength was evaluated through right and left hand-grip strength and the maximal amount of weight that could be lifted when performing biceps curls, bench press, and leg press. The results showed an improvement in the females' muscular strength observed for the right hand-grip, left hand-grip, biceps curls, bench press, and leg press. In addition, the use of water as resistance to improve muscular strength resulted in an increase in peak torque of the knee flexors, knee extensors, and biceps brachii.

Aquatic training has also been evaluated in other populations. Martin (54) performed a study that examined strength gains through aquatic training of the shoulder external rotator in 30 college-aged females. This study consisted of a 6-week aquatic 
exercise program that occurred three times a week. Strength gains were compared to a control group that did not participate in the aquatic exercise program. The results show that the 6-week aquatic training program is able to significantly improve the shoulder external rotators when compared to a control group.

The efficacy of aquatic training was also investigated to examine its effects on increasing muscular strength of the knee extensors and flexors and shoulder abductors and adductors in 18 adults (85). The three day a week 10-week training programs included fourteen females aged from 27 to 31 years. This investigation showed strength improvements occurred in the aquatic trained group over the control group who did not exercise and only continued their normal daily activity.

The low impact nature of aquatic training has increased its use as a preventive and therapeutic modality for rehabilitation, which led to the incorporation of aquatic activities in physical therapy programs. Woods (88) evaluated the physical benefits of an aquatic and traditional rehabilitation program on 18 male patients suffering from low back pain and dysfunction. Each subject participated in eighteen one-hour treatments. The treatment consisted of isolated low back, abdominal, and lower extremity exercises to promote muscular strength. The results indicated that both rehabilitation programs improved strength and flexibility; however, the aquatic rehabilitation patients indicated a reduction in the self-report of pain.

The preceding studies support the inclusion of aquatic activities in training programs to enhance muscular strength $(9,54,84,85)$ while reducing muscle soreness and pain (88). The primary advantage of aquatic activities is the effects of buoyancy and resistance that places minimum strain on the joints and muscles. Aquatics provide twelve 
times the resistance when compared to activities on land, therefore strengthening the muscle with a reduced chance of producing muscle soreness (6).

\section{SUMMARY}

The consensus from the literature is that plyometric training will promote strength and conditioning. Plyometrics represent the bridge between force and power, and are perceived as a method of training that can directly improve competitive performance (17). It has been shown that plyometric training will elicit improvements in power output, force production, and velocity. However, the major concern with plyometric training is the increased risk of muscle soreness and muscle damage. Muscular strength gains have been documented in several aquatic training programs. Research has also demonstrated that the resistance of water is greater than air, which increases the work of movements performed in water. It can be speculated that combining aquatic training with plyometrics may provide a safer, more useful form of sports injury prevention and athletic performance enhancement. 


\section{CHAPTER 3}

\section{METHODS}

\section{SUBJECTS}

This study was approved for the proposed research according to The Ohio State University Institutional Review Board (Appendix A), and all subjects submitted an informed consent form for investigation treatment or procedures according to the University Office of Research Risks Protection (Appendix B). Thirty-two female subjects were recruited from the campus of The Ohio State University through advertisement, informational meetings, and word of mouth. The subjects were between the ages of 18 and 25. The subjects were non-pregnant, healthy, physically active females with some sport experience and no previous history of orthopedic or musculoskeletal injuries that exhibit chronic problems and occurred within the past 6 months. Any individual who had a musculoskeletal injury or leg length discrepancy that would inhibit her ability to participate in a plyometric training program was excluded from the study. Subjects were screened through a series of questionnaires to determine their level of physical activity (Paffenbarger Physical Activity Questionnaire; 64) 
(Appendix C), demographic form (Appendix D), general medical history form (Appendix E), and musculoskeletal history form (Appendix F).

The female subjects were healthy adults between the ages of 18 and 25 . They had been exercising regularly for at least 6 months prior to the study and had been involved in sports (i.e., basketball, crew, cross country, field hockey, golf, gymnastics, lacrosse, rowing, rugby, soccer, softball, swimming, track and field, and volleyball) for an average of five years. Table 3.1 presents demographics characteristics of the subjects at the pretraining $(\mathrm{n}=32)$.

\begin{tabular}{lcc}
\hline \multicolumn{1}{c}{ Characteristics } & Means \pm SEM & Range \\
\hline Age (yr) & $20.2 \pm 0.3$ & $18.0-25.0$ \\
Height $(\mathrm{cm})$ & $168.0 \pm 1.1$ & $155.0-180.0$ \\
Weight (kg) & $68.3 \pm 1.7$ & $51.2-91.6$ \\
Percent body fat (\%) & $27.0 \pm 1.1$ & $13.8-43.6$ \\
Months of regular physical activity (mon) & $58.8 \pm 6.7$ & $6.0-200.0$ \\
Anaerobic activity (min/wks) & $75.0 \pm 5.3$ & $40.0-150.0$ \\
Aerobic activity (min/wks) & $131.7 \pm 7.5$ & $80.0-200.0$ \\
Sport experience (yr) & $5.5 \pm 0.3$ & $3.0-9.0$ \\
\hline
\end{tabular}

Table 3.1 Demographic characteristics for $\mathrm{n}=32$ pre-training. 
The literature consensus is that a high dosage of antioxidants may reduce the amount of membrane damage following exercise (56) and interferes with perceived muscle soreness (45). Therefore, subjects agreed to refrain from taking more than the recommended daily allowance (RDA) of antioxidants during the course of this study. Subjects also consented to refrain from participating in other forms of lower extremity resistance training and any aerobic training during the course of the study. Individuals who consumed ergogenic aids or performance enhancement supplementation were excluded from participating in this study.

\section{PROCEDURE}

The training study took place over 10-weeks. The first week consisted of pretraining performance variables assessment. Week 2 of the study marked the beginning of the plyometric training protocol during which the baseline muscle soreness assessments were obtained. An increase in the plyometric training intensity occurred at the beginning of week 4, and marked the second assessments of muscle soreness. Mid-training performance variables were measured during week 5 of training with the training sessions occurring concurrently. Week 7 marked the second increase in training intensity and included the last assessments of muscle soreness. The plyometric training concluded at the end of week 9 of the study and post-training performance variables were assessed during week 10. A time-line of the training study schedule is located in Appendix G. Pre-, mid-, and post-training performance variables assessments were conducted during week 1, week 5, and week 10. Training assessments included the evaluation of 
performance variables: (1) measurement of peak power output using a Sargent vertical jump test (24); (2) assessment of peak torque through isokinetic strength testing (52); and (3) assessment of peak velocity by using a 40-meter sprint (72). Three assessments of muscle soreness and pain sensitivity were conducted of the rectus femoris, biceps femoris, and gastrocnemius muscles through subjective and objective use of a self-report muscle soreness scale (19) and an algometer (38). Muscle soreness and pain sensitivity were assessed at baseline (first week of training) and when training intensity was increased at week 3 and week 6.

In addition to the above performance variables, pre-training assessments (week 1) also included the evaluation of height, leg length, and body density. Subjects with leg length discrepancy $\geq 3 \mathrm{~cm}$ were excluded from participating in this study. Exclusion for the study was due to the potential pain and discomfort that may be suffered during plyometric training when the body is out of alignment during ground contact. The evaluation of body density also occurred for post-training assessments (week 10).

Familiarization sessions occurred prior to the pre-training assessments to allow subjects to become familiar with the testing procedures and methods. Prior to each testing assessment each subject was given a 5-minute warm-up. 


\section{PLYOMETRIC TRAINING PROTOCOL}

Following the familiarization sessions, the subjects were matched based on the vertical jump prior to the first week of the study and randomly assigned to one of two treatment programs: a land plyometric training program and an aquatic plyometric training program. Subjects were matched based on vertical jump in an attempted to keep the two training groups equivalent in power output. Subjects in the aquatic plyometric training program were provided with aqua shoes to protect their feet.

The plyometric training programs were three days a week for 8-weeks. Each training session lasted 65 minutes, beginning with 15-minutes of warm-up exercises. The warm-up activities concentrated on the specific muscles that would be utilized during the training sessions. The warm-up included a light jog and stretches that increased blood flow the hamstrings, quadriceps, adductors, hip flexors, and calf muscles. The specific warm-up activities are located in Appendix H.

During each session, the training activities lasted 50 minutes and consisted of a series of bounds, hops, and jumps that were specifically designed for the development of explosive power and force production of the knee extensors and knee flexors; rectus femoris and biceps femoris. Descriptions of each drill, training intensity, and training volume are located in Appendix I.

The two training programs were identical except for the training environment; the land training sessions were conducted in a gymnasium and the aquatic training sessions were performed in a pool at the depth of 4 to $4 \frac{1}{2} 2$ feet of water. The temperature of the pool was keep consistent at $77^{\circ} \mathrm{F}\left(25^{\circ} \mathrm{C}\right)$ to $79^{\circ} \mathrm{F}\left(26^{\circ} \mathrm{C}\right)$ based on a daily log kept by 
the pool facility manager. It is recommended that maximum water temperature should range between $79^{\circ} \mathrm{F}\left(26^{\circ} \mathrm{C}\right)$ and $82^{\circ} \mathrm{F}\left(28^{\circ} \mathrm{C}\right)$ to prevent any heat related complications or illnesses (80). Each training session was instructed by the same research assistants to ensure consistency.

\section{MEASUREMENTS}

\section{PEAK POWER OUTPUT}

The Sargent vertical jump test was used to assess peak power production. The vertical jump test is often used as an indicator of "explosiveness" in physical fitness testing (27), and the vertical jump test provides an effective measurement of power output (48). This test was also selected due to its validity and reliability for predicting power output (24).

The Sargent test involves measuring the difference between a person's standing reach and the height recorded from a jump and reach (48). The subject placed one hand in white chalk, and then recorded her standing reach by extending that arm up, while facing the vertical jump board. Next, the subject was instructed to keep her feet flat on the ground. The subject then stood sideways, squatted to a 90 degree angle, and exploded up vertically as high as possible using her arms and legs to help project her body upwards. Subjects were instructed to touch the wall at the highest point of the jump. The difference between the standing height and the jump height is the vertical jump value. Three two-foot squat jumps were completed with a 1-minute break to ensure 
full recovery between jumps. The same arm was used for each vertical jump assessment. Both peak and average vertical jump values were recorded in centimeters $(\mathrm{cm})$. The detailed Sargent vertical jump testing protocol and procedures are located in Appendix J.

\section{PEAK TORQUE PRODUCTION}

Peak torque production was assessed using isokinetic strength testing. Isokinetic testing was chosen due to its validity (52), reliability $(r=0.99)(62)$, and consistency for ensuring an accurate measurement (52). Isometric testing or one repetition maximum (1 RM) does not provide high reliability and is often characterized as a learned process (52). Isokinetic testing also accounts for the velocity of movement that is uncontrolled and difficult to measure with free weights and machines (49).

The knee extensors and knee flexors of each subject's dominant leg were tested for peak torque pliometrically (i.e., eccentric contraction) and miometrically (i.e., miometric contraction) at $60^{\circ} / \mathrm{sec}$; values were recorded in Newton-meters $(\mathrm{N} \cdot \mathrm{m})$. When testing the knee extensors and knee flexors, the ankle, thigh, hip or waist, and chest were stabilized through Velcro straps. The non-tested or non-dominant leg was not stabilized. The position of the powerhead height, seat distance, seat angle, and ankle lever arm were recorded and used for each subject to improve the reliability of testing for subsequent training assessments. The test consisted of two sets and a warm-up set with a 2-minute rest period between each set to ensure a full recovery. The specific isokinetic testing protocol and procedures are located in Appendix K. 


\section{VELOCITY}

Velocity was assessed using a 40-m sprint and measured in meters per seconds (m

$\left.\cdot \mathrm{s}^{-1}\right)$. A 40-m sprint was selected because it is widely used as a measure of velocity, and sprint tests that measure distances greater than $60-\mathrm{m}$ are not true indicators of speed due to the influence of running skill and technique (72). Each subject was instructed to perform 3 maximal effort sprints from a 3-point stance position on a hard even surface in an indoor facility. The subjects were instructed to position the front of either the right or left foot on the start line and to touch the same line with either the left hand, if the right foot was the lead foot, or the right hand if the left foot was the lead foot. The subjects were instructed to rise up on the push-off foot, in order to get more push-off of the ground and to "sprint as fast as possible."

Two research assistants recorded velocity and the values were averaged and reported in seconds (s). Both the peak (fastest) and average time were recorded. Each of the three sprints was separated by a 3-minute rest period to ensure full recovery between sprints. The specific 40-meter sprint testing protocol and procedures are located in Appendix L. 


\section{MUSCLE SORENESS}

Muscle soreness of the rectus femoris, biceps femoris, and gastrocnemius muscles was assessed using an established self-report ordinal scale ranging from 1 (no soreness) to 10 (very, very sore) (19). This scale has been previously used in other muscle soreness investigations (e.g., 19, 43). The muscle soreness scale was modified by inserting a picture of each specific muscle. Subjects were asked to rate the soreness of each individual muscle subjectively. Muscle soreness assessments occurred during each increase in training intensity. Muscle soreness was assessed at baseline (first week of training) and when training intensity was increased at week 3 and week 6 at 0, 48, and 96 hr post-training bout. A copy of the muscle soreness scales and the specific protocol is located in Appendix M.

\section{PAIN SENSITIVITY}

The algometer (Algometer FDK40, Scrip Inc.; Peoria, IL) is a pressure gauge that has been successfully employed for the assessment of general sensitivity to pain in normal tissue $(26,47)$. Pressure gauges have been used to evaluate pain sensitivity in numerous clinical and physiological research studies $(14,51,58,59,89)$. With algometers, pressure is applied through a rubber disc to a specific surface on the body corresponding to the target muscle or area An algometer was incorporated into this study to give more precise assessment of pain sensitivity and discomfort in the rectus femoris, biceps femoris, and gastrocnemius muscles. 
The pain sensitivity to discomfort was taken from the mid-point of each specific muscle (i.e., rectus femoris, biceps femoris, and gastrocnemius) of the subjects' dominant leg. The anatomical point of measurement was recorded with a tape measure from anatomical references and used during each assessment. The subjects were in the prone position and were ask to keep their muscle relaxed during the pressure tolerance assessment. Pressure increased and was applied at a continuous rate, about $1 \mathrm{~kg} / \mathrm{sec}$ by a research technician. The algometer ranges from a low of $2 \mathrm{~kg}$ (extreme pain and sensitivity) to a high of $20 \mathrm{~kg}$ (no pain and sensitivity) in 200 gram increments. The subjects were instructed to say "stop" or "now" when they felt the onset of discomfort and/or pain. Pressure sensitivity was chosen as the marker for pain sensitivity due to the influence of pre-existing factors (e.g., pain tolerance) on maximum pressure thresholds. Pain sensitivity was assessed at baseline (first week of training) and when training intensity was increased at week 3 and week 6 at 0,48 , and $96 \mathrm{hr}$ post-training bout. The specific algometer protocol and a diagram that illustrates the point of pressure tolerance assessment are located in Appendix N. 


\section{BODY COMPOSITION ASSESSMENT}

Body composition was assessed through the use of air-displacement plethysmograph (25). The Bod Pod Body Composition System (Bod Pod, Life Measurement Instruments; Concord, CA) uses whole body densitometry to determine body density (percent body fat and lean body mass). Once the overall body density is known, the relative proportion of body fat and lean body mass can be calculated through the use of scientific equations that relate body density to body fat and lean tissue (25). The commonly used equation, which translates whole body density to percent body fat, is the Siri equation:

$$
\text { Percent Fat }=[495 / \text { Density }]-450
$$

Once the percent body fat is calculated, the percent lean body mass can also be determining as follows:

\section{Percent Lean Body Mass $=100-$ Percent Fat}

The Bod Pod uses air instead of water to measure body volume (82). It is based on the application of Boyle's Law, which states volume and pressure vary inversely with one another. That is as volumes increases, pressure decreases and as volume decreases, pressure increases (25):

$$
\mathrm{P} 1 \mathrm{~V} 1=\mathrm{P} 2 \mathrm{~V} 2=\text { Constant }
$$

The Bod Pod instrument has been validated against hydrostatic weighing to estimate body density (55). McCrory et al. reported that the Bod Pod is a highly reliable $(\mathrm{r}=0.93)$ method for determining body density in comparison to hydrostatic weighing (55). This method of body density assessment holds several advantages over hydrostatic 
weighing in that it is quick, relative simple to operate, and may be able to accommodate special populations such as the obese, elderly, and disabled (55). The specific protocol for the body density assessment is located in Appendix O.

\section{ANTHROPOMETRIC ASSESSMENTS}

Anthropometric assessments were used to determine standing height and leg length. Height was measured without shoes using a height and weight scale and reported in $\mathrm{cm}$. Leg length measurements were used to detect leg length discrepancies. Leg length measurements were taken from the greater trochanter of the femur to the lateral malleous of the fibula of the right and left leg. The specific protocol for the anthropometric assessment is located in Appendix P.

\section{STATISTICAL ANALYSIS}

Data were analyzed using the Statistical Package for the Social Sciences (SPSS) Version 11.0 for Windows ${ }^{\mathrm{TM}}$ (SPSS Inc. 1989-2001). Findings were examined in two sections; performance outcome variables (vertical jump, peak torque, and velocity) and muscle soreness (muscle soreness scale and pain sensitivity using an algometer). Both descriptive and inferential statistics were used to describe and draw conclusions related to the improvements in the performance enhancement outcome variables and muscle soreness to test the hypotheses regarding the relationship between the independent variables [training group: land plyometrics versus aquatic plyometrics] on performance 
enhancement and muscle soreness. To determine if land plyometrics and aquatic plyometrics differed on their pre-training, dependent variables were compared using an independent - samples $t$ test.

A $2 \times 3$ repeated measures multivariate analysis of variance (MANOVA) with Wilks' lambda post hocs testing was used to test the hypothesis of performance improvement outcome across all dependent variables for both levels of the independent variable, land plyometrics and aquatic plyometrics. A multivariate question examining the combination of all the performance variables was used because the performance variables were all an attempt to describe performance in general rather than an attempt to describe specific performance attributes. A $2 \times 3 \times 3 \times 3$ repeated measures multivariate analysis of variance (MANOVA) was used to test the hypothesis of muscle soreness outcomes across all dependent variables for both levels of the independent variable, land plyometrics and aquatic plyometrics. A univariate approach was taken for the muscle soreness assessment because each instrument represented a different aspect of muscle soreness.

All statistical comparison was set at $p \leq 0.05$, and values are expressed as means \pm standard error of the mean (SEM). 


\section{CHAPTER 4}

\section{RESULTS}

Thirty-two college-aged females were recruited from the campus of The Ohio State University to participate in this study. Thirty-one females completed the study. One subject dropped out and was unable to complete the study due to a medical condition. Study results are presented in two sections according to the purpose of the investigation. The first section provides findings pertaining to the performance outcome research questions and hypotheses, and the second section illustrates the findings concerning muscle soreness represented as a muscle soreness scale and pain sensitivity. All statistical comparisons were set at $p \leq 0.05$ and values are expressed as means \pm standard error of the mean (SEM). 
Independent samples t-tests revealed that subjects' characteristics were similar at the beginning of the study with and without the inclusion of data from the dropout subject. The measured characteristics of the two treatment groups were similar at the beginning of the study (Table 4.1).

\begin{tabular}{lcc}
\hline Characteristics & $\begin{array}{c}\text { Land plyometrics } \\
(\mathrm{n}=15)\end{array}$ & $\begin{array}{c}\text { Aquatic plyometrics } \\
(\mathrm{n}=16)\end{array}$ \\
\hline Age (yr) & $20.6 \pm 0.6$ & $19.8 \pm 0.3$ \\
Height (cm) & $167.2 \pm 1.6$ & $168.6 \pm 1.5$ \\
Weight (kg) & $68.5 \pm 2.3$ & $66.8 \pm 2.1$ \\
Percent body fat (\%) & $27.3 \pm 1.5$ & $26.1 \pm 1.7$ \\
Regular physical activity (months) & $63.5 \pm 7.9$ & $73.4 \pm 7.9$ \\
Anaerobic activity (min/wks) & $78.7 \pm 7.5$ & $131.6 \pm 7.6$ \\
Aerobic activity (min/wks) & $112.0 \pm 8.0$ & $5.9 \pm 0.3$ \\
Sport experience (yr) & $5.3 \pm 0.5$ & \\
\hline
\end{tabular}

Table 4.1 Descriptive statistics for plyometric treatments groups $(n=31)$ at pre-training. 


\section{PERFORMANCE OUTCOMES}

In investigating performance, global performance was examined by the multivariate linear combination of all the performance dependent variables, as well as looking at each performance variable independently through a univariate analysis. In the multivariate analysis, there was a multivariate main effect for time, $\left(\mathrm{F}_{(12,18)}=229.1, \mathrm{P} \leq\right.$ $0.001,1-\beta=1.0)$. Post-hoc testing of the time effect revealed that pre-training $<$ midtraining < post-training. There was no main effect for treatment $\left(\mathrm{F}_{(6,24)}=0.6, \mathrm{P}=0.724\right.$, $1-\beta=0.196)$ and no treatment by time interaction effect $\left(\mathrm{F}_{(12,18)}=1.134, \mathrm{P}=0.39,1-\right.$ $\beta=0.42$ ) indicating that there was no performance outcome difference between the land and aquatic trained groups.

In addition to the multivariate performance analysis, an examination of each performance variable univariately was completed. The following sections describe the univariate portions of the performance MANOVA. Performance outcome variables included peak power output [vertical jump distance in $\mathrm{cm}$ using a vertical (squat jump) jump test], peak torque production [torque in $\mathrm{N} \cdot \mathrm{m}$ using BIODEX isokinetic testing], and velocity $\left[\mathrm{m} \cdot \mathrm{s}^{-1}\right.$ in a 40-meter sprint] at pre-training, mid-training (week 5), and post-training. 
Peak power output was similar for both groups across training as assessed by a Sargent vertical jump test and presented as standing jump height in $\mathrm{cm}$ (Table 4.2).

\begin{tabular}{lcc}
\hline \multicolumn{1}{c}{ Peak vertical jump $(\mathrm{cm})$} & $\begin{array}{c}\text { Land plyometrics } \\
(\mathrm{n}=15)\end{array}$ & $\begin{array}{c}\text { Aquatic plyometrics } \\
(\mathrm{n}=16)\end{array}$ \\
\hline Pre-training & $32.6 \pm 1.7$ & $31.9 \pm 1.6$ \\
Mid-training & $35.6 \pm 1.6 *$ & $36.7 \pm 1.8 *$ \\
Post-training & $43.2 \pm 1.7+$ & $42.6 \pm 1.9+$ \\
\hline
\end{tabular}

$*$ Pre-training $<$ mid-training, $p \leq 0.001$.

+ Mid-training $<$ post-training, $p \leq 0.001$.

Table 4.2 Effects of plyometric training on peak vertical jump (cm).

Regardless of training group, there was a significant increase in peak vertical jump (peak power output) overtime, $p \leq 0.001$. Post-hoc contrasts revealed that there was a significant increase in peak vertical jump (peak power output) from pre-training to mid-training, $p \leq 0.001$, and from mid-training to post-training, $p \leq 0.001$ (Table 4.2). 
Peak torque productions were measured miometrically and pliometrically at $60 \%$ sec. Peak torque production was similar for both groups across training as assessed through isokinetic testing (Table 4.3 and 4.4).

\begin{tabular}{lcc}
\hline $\begin{array}{l}\text { Peak torque production }(\mathrm{N} \cdot \mathrm{m}) \\
60 \% \text { sec }- \text { Miometric }\end{array}$ & $\begin{array}{c}\text { Land plyometrics } \\
\text { Extension }\end{array}$ & $\begin{array}{c}\text { Aquatic plyometrics } \\
(\mathrm{n}=15)\end{array}$ \\
\hline \multicolumn{1}{c}{ Pre-training } & $151.0 \pm 7.2$ & $161.0 \pm 5.2$ \\
Mid-training & $162.0 \pm 7.3 *$ & $175.0 \pm 5.5 *$ \\
Post-training & $189.0 \pm 6.8+$ & $201.0 \pm 5.4+$ \\
\multicolumn{1}{c}{ Flexion } & $82.7 \pm 2.9$ & $86.3 \pm 3.4$ \\
Pre-training & $90.1 \pm 4.41 *$ & $96.7 \pm 3.2 *$ \\
Mid-training & $120.0 \pm 4.2+$ & $125.0 \pm 3.1+$ \\
Post-training & & \\
\hline
\end{tabular}

$*$ Pre- training $<$ mid-training, $p \leq 0.001$.

+ Mid-training $<$ post-training, $p \leq 0.001$.

Table 4.3 Effects of plyometric training on peak torque production miometrically.

Regardless of training group, peak torque production (miometric-extension and miometric-flexion) increased significantly overtime. Post hoc contrasts revealed that there was a significant increase in peak torque miometrically from pre-training to midtraining, $p \leq 0.001$, from mid-training to post-training, $p \leq 0.001$ (Table 4.3). 


\begin{tabular}{lcc}
\hline $\begin{array}{l}\text { Peak torque production }(\mathrm{N} \cdot \mathrm{m}) \\
60 \% \text { sec - Pliometric }\end{array}$ & $\begin{array}{c}\text { Land plyometrics } \\
(\mathrm{n}=15)\end{array}$ & $\begin{array}{c}\text { Aquatic plyometrics } \\
(\mathrm{n}=16)\end{array}$ \\
\hline \multicolumn{1}{c}{ Extension } & $94.8 \pm 3.9$ & $96.2 \pm 3.3$ \\
Pre-training & $108.0 \pm 4.6 *$ & $119.0 \pm 4.7 *$ \\
Mid-training & $137.0 \pm 4.8+$ & $147.0 \pm 4.9+$ \\
Post-training & & \\
$\quad$ Flexion & $185.0 \pm 9.3$ & $188.0 \pm 7.2$ \\
Pre-training & $202.0 \pm 10.0 *$ & $209.0 \pm 6.1 *$ \\
Mid-training & $230.0 \pm 10.3+$ & $235.0 \pm 6.2+$ \\
Post-training & & \\
\hline
\end{tabular}

* Pre-training $<$ mid-training, $p \leq 0.001$.

+ Mid-training $<$ post-training, $p \leq 0.001$.

Table 4.4 Effects of plyometric training on peak torque production pliometrically.

Regardless of training group, peak torque production (pliometric-extension and pliometric-flexion) increased significantly overtime. Post hoc contrasts revealed that there was a significant increase in peak torque pliometrically from pre-training to midtraining, $p \leq 0.001$, from mid-training to post-training, $p \leq 0.001$ (Table 4.4). 
Velocity was measured using a 40-m sprint. There were no differences between the two groups at each measurement (Table 4.5).

\begin{tabular}{lcc}
\hline \multicolumn{1}{c}{\begin{tabular}{l} 
Velocity 40 -m sprint \\
\multicolumn{1}{c}{$\left(\mathrm{m} \cdot \mathrm{s}^{-1}\right)$}
\end{tabular}} & $\begin{array}{c}\text { Land plyometrics } \\
(\mathrm{n}=15)\end{array}$ & $\begin{array}{c}\text { Aquatic plyometrics } \\
(\mathrm{n}=16)\end{array}$ \\
\hline Pre-training & $5.97 \pm 285.71$ & $6.15 \pm 333.33$ \\
Mid-training & $6.15 \pm 307.69 *$ & $6.35 \pm 400.00 *$ \\
Post-training & $6.35 \pm 307.69+$ & $6.56 \pm 400.00+$ \\
\hline
\end{tabular}

* Pre-training $<$ mid-training, $p \leq 0.001$.

+ Mid-training $<$ post-training, $p \leq 0.001$.

Table 4.5 Effects of plyometric training on velocity 40-m sprint.

Regardless of training group, velocity $\left(\mathrm{m} \cdot \mathrm{s}^{-1}\right)$ improved significantly overtime.

This improvement reflects less time to complete a 40-m sprint. Post hoc contrasts revealed that there was a significant increase in velocity from pre-training to midtraining, $p \leq 0.001$, from mid-training to post-training, $p \leq 0.001$ (Table 4.5). 


\section{MUSCLE SORENESS}

For the muscle soreness question, each dependent variable was tested

univariately. Muscle soreness and pain sensitivity were assessed at baseline (first week of training) and when training intensity was increased at week 3 and week 6 at 0,48 , and $96 \mathrm{hr}$ post-training bout.

\section{$\underline{\text { Muscle Soreness Scale }}$}

Muscle soreness was similar for both groups at baseline (first week of training); however, the land plyometric training group had a significantly higher perception of muscle soreness for the rectus femoris, biceps femoris, and gastrocnemius muscles at 48 $\mathrm{hr}$ and $96 \mathrm{hr}$ after the training bout. In examining the four-way interaction of treatment group by week by time by muscle, a significant difference was observed in muscle soreness perception $\left(F_{(8,232)}=2.6, P=0.01,1-\beta=0.92\right)$. This 4-way interaction is broken down in figures $4.1-4.3$.

For the rectus femoris muscle (Figure 4.1), during the baseline assessment, the land plyometric training group had a significantly greater perception of muscle soreness than the aquatic plyometric training group at $48 \mathrm{hr}$ and $96 \mathrm{hr}$ post-training bout. At the first increase in training intensity, land plyometrics muscle soreness perception was greater than aquatic plyometrics at $48 \mathrm{hr}$ and $96 \mathrm{hr}$ post-training bout. Finally, at the second increase in training intensity, the land plyometrics had a greater perception of muscle soreness than aquatic plyometrics at $48 \mathrm{hr}$ and $96 \mathrm{hr}$ post-training bout. A numerical data table is located in Appendix Q that illustrates the rectus femoris muscle soreness at each measurement point. 


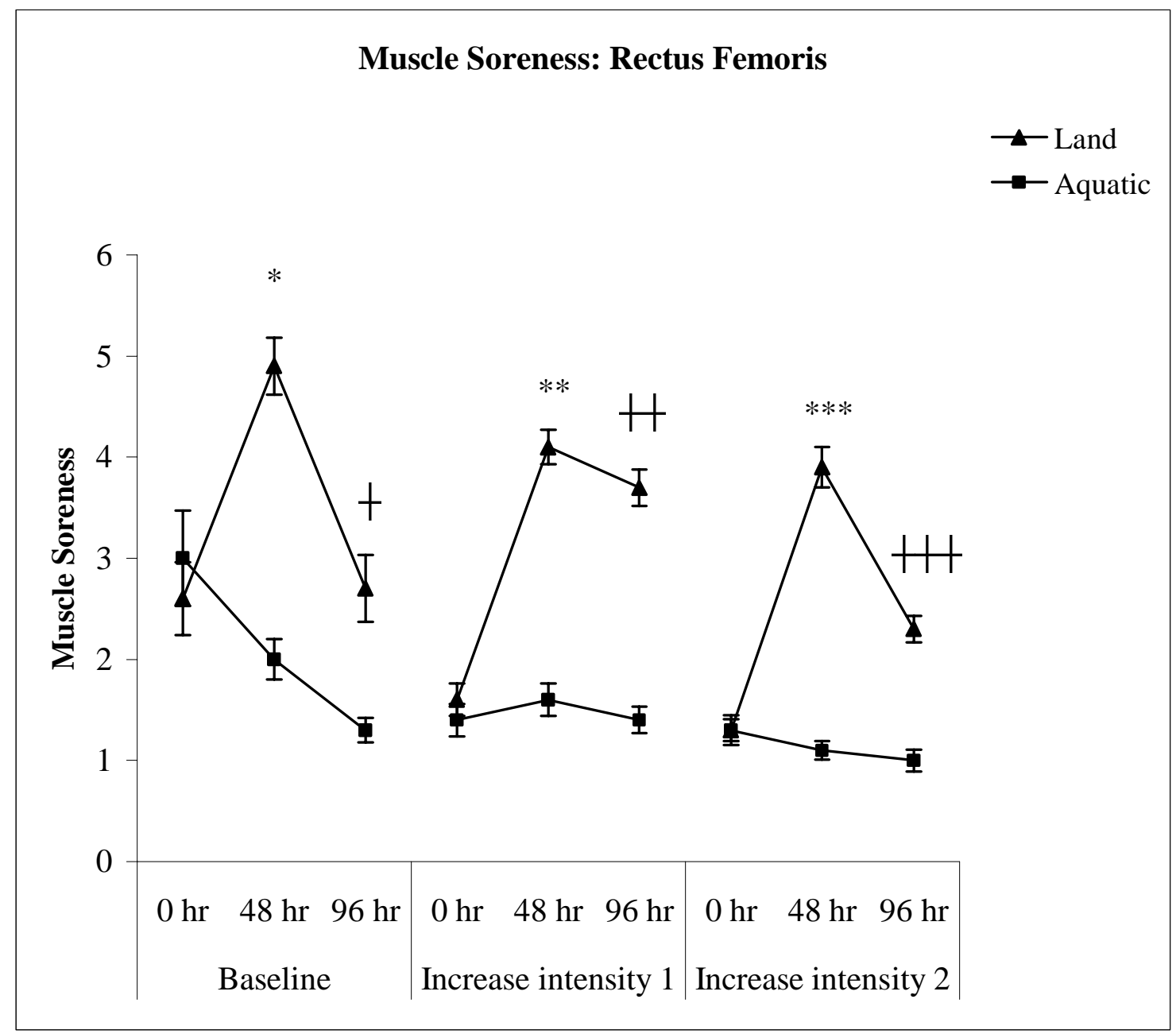

* Baseline: $48 \mathrm{hr}$ land plyometrics $>$ aquatic plyometrics, $p \leq 0.001$.

十 Baseline: $96 \mathrm{hr}$ land plyometrics $>$ aquatic plyometrics, $p \leq 0.001$.

$* *$ Increase in training intensity 1: $48 \mathrm{hr}$ land plyometrics > aquatic plyometrics, $p \leq$ 0.001 .

H Increase in training intensity 1: $96 \mathrm{hr}$ land plyometrics $>$ aquatic plyometrics, $p \leq$ 0.001 .

*** Increase in training intensity 2: $48 \mathrm{hr}$ land plyometrics $>$ aquatic plyometrics, $p \leq$ 0.001 .

H Increase in training intensity 2: $96 \mathrm{hr}$ land plyometrics > aquatic plyometrics, $p \leq$ 0.001 .

Figure 4.1 Differences in muscle soreness between land and aquatic plyometric training groups: rectus femoris. 
For the biceps femoris muscle (Figure 4.2), during the baseline assessment (first week of training), the land plyometric training group had a significantly greater perception of muscle soreness than the aquatic plyometric training group at $48 \mathrm{hr}$ and 96 hr post-training bout. At the first increase in training intensity, land plyometrics muscle soreness perception was greater than aquatic plyometrics at $48 \mathrm{hr}$ and $96 \mathrm{hr}$ post-training bout. Finally, at the second increase in training intensity, the land plyometrics had a greater perception of muscle soreness than aquatic plyometrics at $48 \mathrm{hr}$ and $96 \mathrm{hr}$ posttraining bout. A numerical data table is located in Appendix $\mathrm{R}$ that illustrates the biceps femoris muscle soreness at each measurement point. 


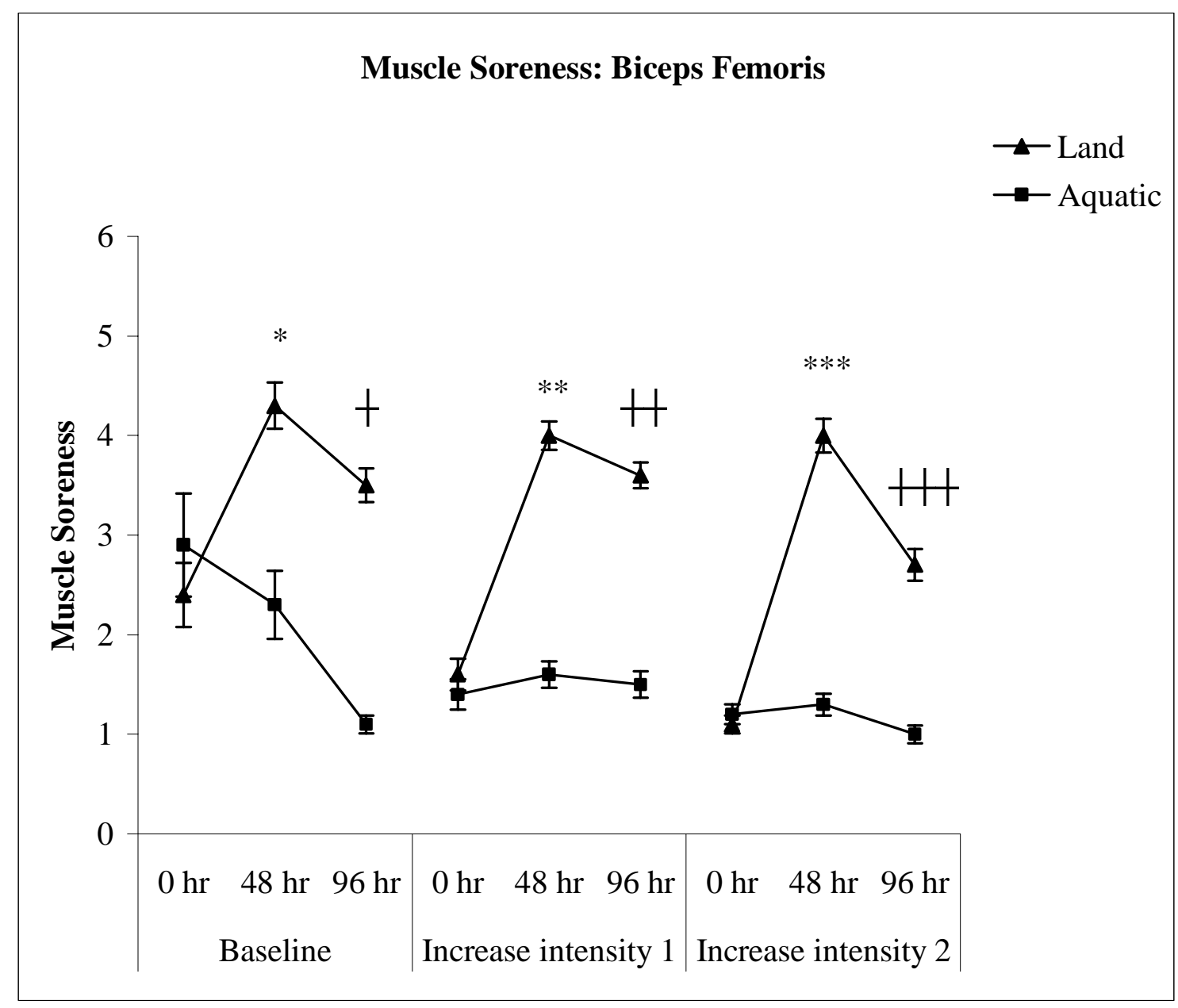

* Baseline: $48 \mathrm{hr}$ land plyometrics $>$ aquatic plyometrics, $p \leq 0.001$.

十 Baseline: $96 \mathrm{hr}$ land plyometrics $>$ aquatic plyometrics, $p \leq 0.001$.

** Increase in training intensity 1: $48 \mathrm{hr}$ land plyometrics > aquatic plyometrics, $p \leq$ 0.001 .

H Increase in training intensity 1: $96 \mathrm{hr}$ land plyometrics $>$ aquatic plyometrics, $p \leq$ 0.001 .

*** Increase in training intensity 2: $48 \mathrm{hr}$ land plyometrics $>$ aquatic plyometrics, $p \leq$ 0.001 .

H Increase in training intensity 2: $96 \mathrm{hr}$ land plyometrics > aquatic plyometrics, $p \leq$ 0.001 .

Figure 4.2 Differences in muscle soreness between land and aquatic plyometric training groups: biceps femoris. 
For the gastrocnemius muscle (Figure 4.3), during the baseline assessment (first week of training), the land plyometric training group had a significantly greater perception of muscle soreness than the aquatic plyometric training group at $48 \mathrm{hr}$ and 96 hr post-training bout. At the first increase in training intensity, land plyometrics muscle soreness perception was greater than aquatic plyometrics at $48 \mathrm{hr}$ and $96 \mathrm{hr}$ post-training bout. Finally, at the second increase in training intensity, the land plyometrics had a greater perception of muscle soreness than aquatic plyometrics at $48 \mathrm{hr}$ and $96 \mathrm{hr}$ posttraining bout. A numerical data table is located in Appendix S that illustrates the gastrocnemius muscle soreness at each measurement point. 


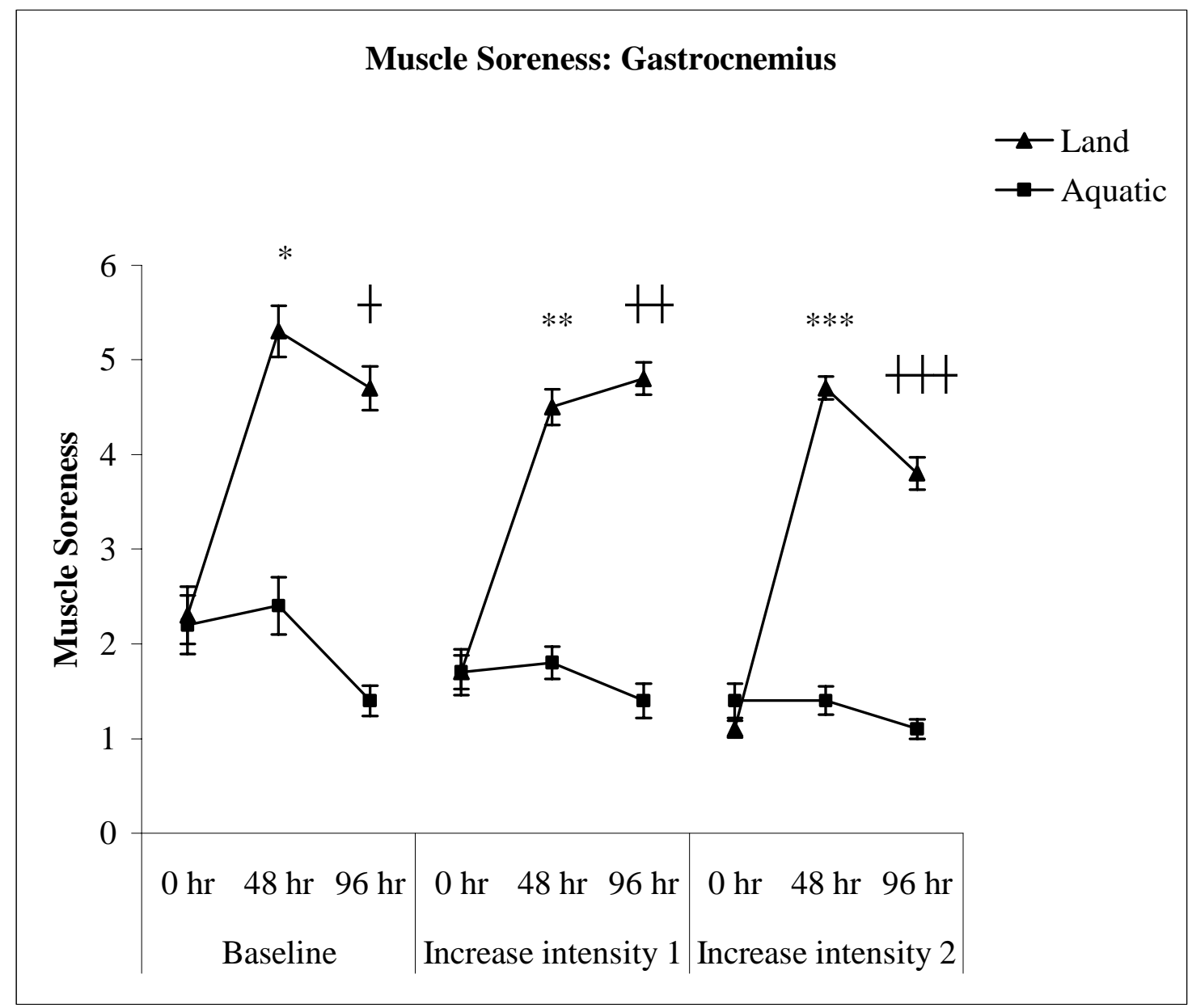

* Baseline: $48 \mathrm{hr}$ land plyometrics $>$ aquatic plyometrics, $p \leq 0.001$.

十 Baseline: $96 \mathrm{hr}$ land plyometrics $>$ aquatic plyometrics, $p \leq 0.001$.

** Increase in training intensity 1: $48 \mathrm{hr}$ land plyometrics > aquatic plyometrics, $p \leq$ 0.001 .

H Increase in training intensity 1: $96 \mathrm{hr}$ land plyometrics $>$ aquatic plyometrics, $p \leq$ 0.001 .

*** Increase in training intensity 2: $48 \mathrm{hr}$ land plyometrics $>$ aquatic plyometrics, $p \leq$ 0.001 .

+ H Increase in training intensity 2: $96 \mathrm{hr}$ land plyometrics $>$ aquatic plyometrics, $p \leq$ 0.001 .

Figure 4.3 Differences in muscle soreness between land and aquatic plyometric training groups: gastrocnemius. 


\section{Pain Sensitivity}

The following sections describe the univariate portion of pain sensitivity. Pain sensitivity was assessed at baseline (first week of training) and when training intensity was increased at week 3 and week 6 at 0,48 , and $96 \mathrm{hr}$ post-training bout. The algometer measures pain sensitivity by the amount of pressure that induces pain and discomfort; higher values indicate less sensitivity (less pain) and lower values indicate more sensitivity (more pain). In examining the four-way interaction of treatment group by week by time by muscle, no significant difference in pain sensitivity were observed $\left(\mathrm{F}_{(8}\right.$, 232) $=1.5, \mathrm{P}=0.15,1-\beta=0.67)$. This 4 -way interaction is broken down in figures $4.4-$ 4.6. There was a significant increase in the perception of pain sensitivity for all muscle sites (rectus femoris, biceps femoris, gastrocnemius) in the land plyometric training group from $0 \mathrm{hr}$ to $48 \mathrm{hr}$ post-training bout at baseline, and when training intensity was increased at week 3 and week $6, p \leq 0.001$; however there was no significant difference in pain sensitivity between the land plyometric and aquatic plyometric training group. Numerical data table of pain sensitivity are located in Appendix T, Appendix U, and Appendix V. 


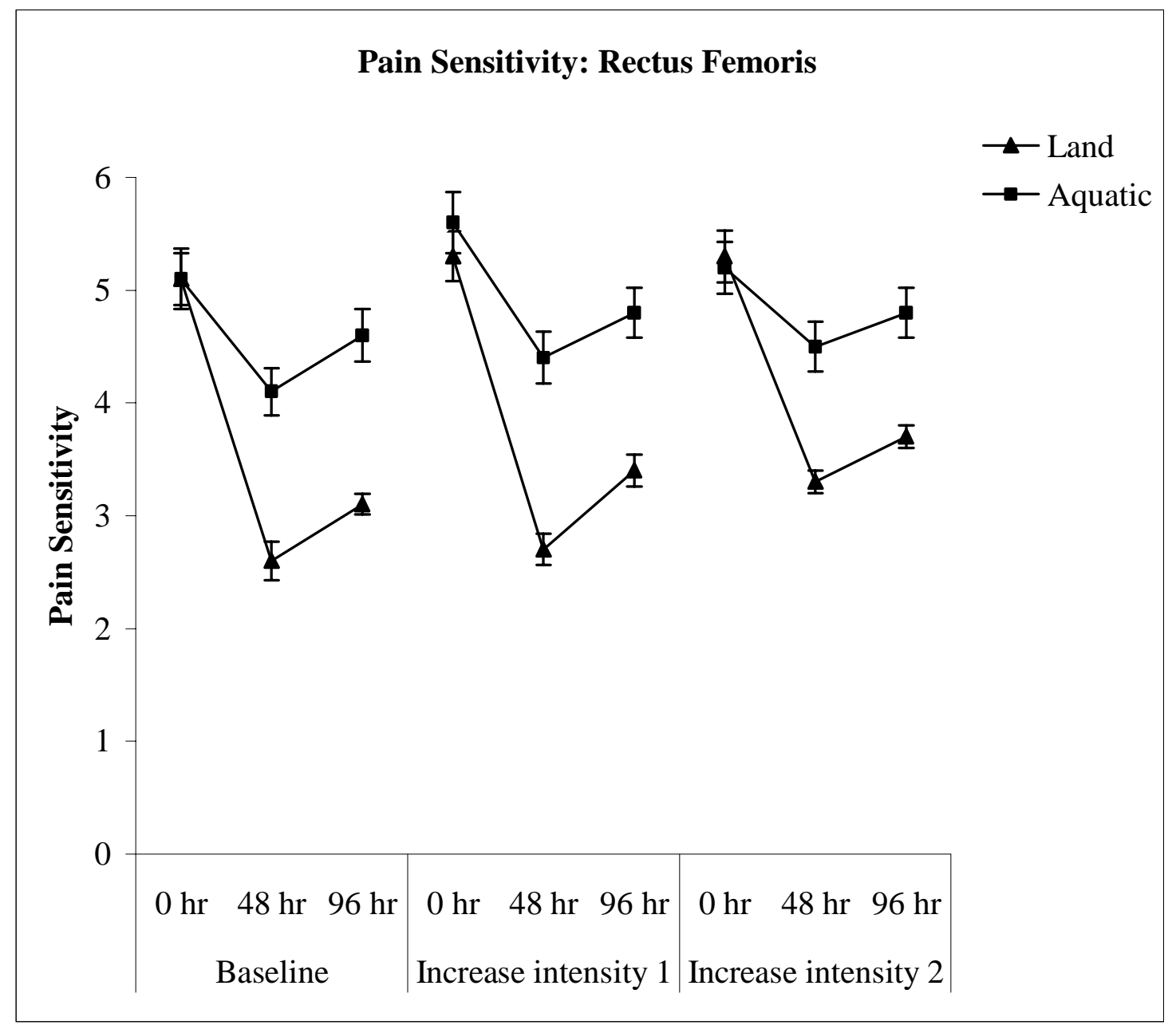

Figure 4.4 Differences in pain sensitivity between land and aquatic plyometric training: rectus femoris. 


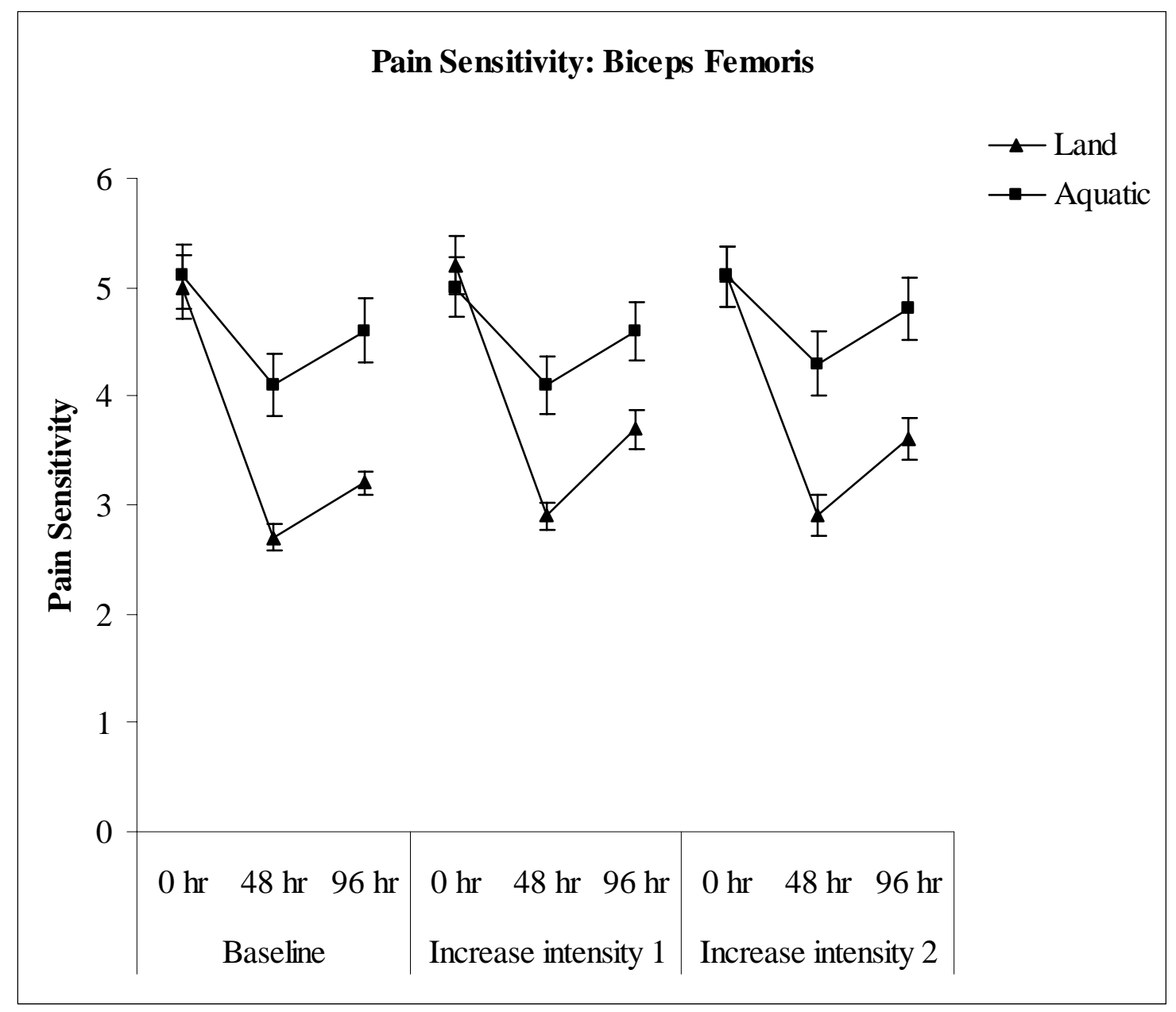

Figure 4.5 Differences in pain sensitivity between land and aquatic plyometric training: biceps femoris. 


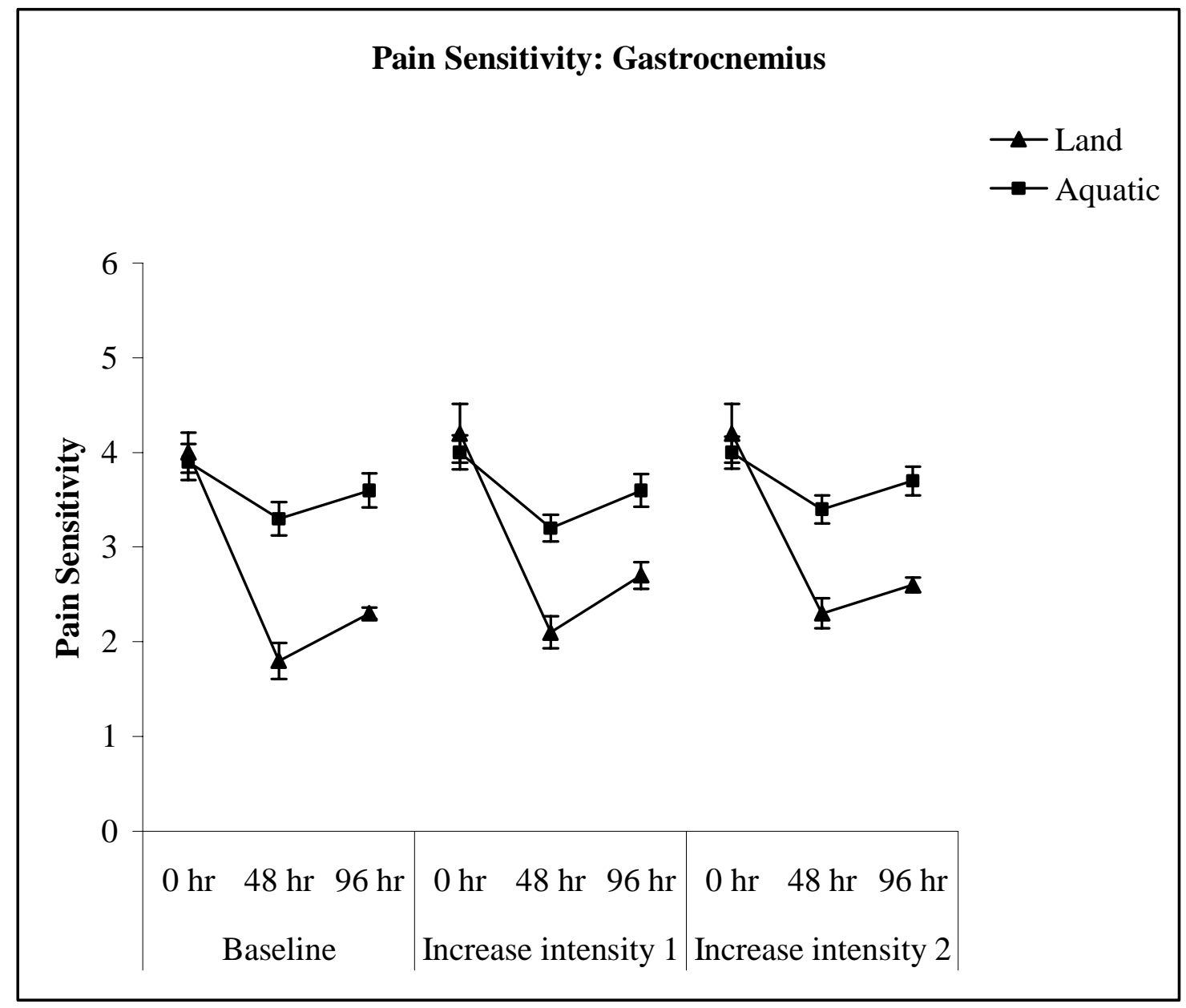

Figure 4.6 Differences in pain sensitivity between land and aquatic plyometric training: gastrocnemius. 


\section{CHAPTER 5}

\section{DISCUSSION}

The past two decades have seen remarkable performances in women's sports. Female athletes are breaking world records with monotonous regularity. Physiologically, the body has been operating the same way. So, what accounts for these phenomenal performances, which in many cases have been deemed physically impossible? Part of the answer is performance enhancement training based on controlled empirical research.

Plyometric training is a form of explosive, physical training that is used to enhance power output, force production, and velocity. Plyometrics comprise an extremely wide range of conditioning exercises that are a subset of the stretch-shortening cycle. The stretch-shortening cycle occurs when a muscle lengthens and then immediately performs a shortening contraction. Stretch-shortening cycles are an integral component of nearly all athletic movements from the explosive and ballistic movements performed during sprinting to performing a snatch in weightlifting. The stretchshortening cycle enhances both movement effectiveness and performance levels by utilizing stored elastic energy within a muscle. Nearly all coaches incorporate plyometric training into conditioning programs to enhance explosive power and speed performance by improving both efficiency and the rate of muscular activation. 
The purpose of this study was to determine the effects of land versus aquatic plyometric training on power, torque, velocity, and muscle soreness in college-aged females. The objectives of the study were (a) to compare changes in power, torque, and velocity between land and aquatic plyometric training programs and (b) to compare degree of muscle soreness overtime between land and aquatic plyometric training groups.

It was hypothesized that land plyometrics and aquatic plyometrics training would result in similar improvements in the measured physiological attributes and that land plyometrics would result in a greater degree of muscle soreness than aquatic plyometrics. The first hypothesis relating to performance outcome was supported since no significant difference was found between the two treatments in any of the variables. Regardless of training environment, there was a significant improvement in power output, peak torque production, and velocity from pre-training to mid-training and mid-training to posttraining.

Previous studies have investigated plyometric training on land and have demonstrated a significant improvement in power output, force production, and velocity in college-aged males $(2,7,10,21,22,24,63,66,69,86)$. This was the first study to examine the effects of aquatic plyometrics on power, torque, and velocity. It is also one of the first plyometric training studies to incorporate female subjects. The results of this study indicated that the aquatic plyometric training program can provide similar improvements in power output, peak torque, and velocity as the traditional land plyometric training program.

Recommendations for future plyometric training studies include increasing the length of the training program. Research has not yet determined the optimal plyometric 
training program length. Brown et al. (12), Clutch et al. (21), and Hiserman (37) have recommended plyometric programs extend 10 to 16 weeks. This study was performed over the course of 10 weeks due to the length of the university's academic term (10 weeks). The actual plyometric training sessions occurred for 8-weeks due to the time needed to pre- and post-test the subjects. A longer training period will allow for more time to progress in intensity and workload as well as longer rest period between sessions. In general, plyometric training programs should be prescribed similarly to both resistance and aerobic training programs (35).

The second purpose of this study was to compare muscle soreness that is associated with land plyometrics and aquatic plyometrics. It was hypothesized that the land plyometric training group would have a greater degree of muscle soreness when compared with the aquatic plyometric training group. Previous research has suggested that land plyometrics carry an increased risk of muscle soreness due to the forces generated during ground impact and the intense pliometric contraction. Jamurtas et al. (43) evaluated the effects of delayed-onset muscle soreness and plasma creatine kinase levels in a plyometric, pliometric, and miometric exercise program. The results indicated that the plyometric- and pliometric-trained groups had a higher perception of delayedonset muscle soreness. Creatine kinase levels were similar for all subjects, but exercise history (i.e., inactive subjects) may have influenced their findings. Research has also concluded that pliometric contractions results in severe muscle soreness upon palpation (74), temporary loss of the exercised muscle's range of motion $(18,74)$, intramuscular swelling and edema $(18,31)$, and a decline in force production (18). 
This study evaluated muscle soreness of the rectus femoris, biceps femoris, and gastrocnemius muscles between the land and aquatic plyometric groups. Muscle soreness was measured through a muscle soreness scale and pain sensitivity with an algometer. The results indicated a significantly higher perception of muscle soreness when assessed with the muscle soreness scale in the land plyometric trained group when compared to the aquatic plyometric trained group for each muscle at $48 \mathrm{hr}$ and $96 \mathrm{hr}$ after training.

There was not a significant difference in pain sensitivity between the two groups. In the pain sensitivity data, we see what appears to be between group differences, yet the MANOVA failed to detect any difference at the 0.05 level. The relative low $\mathrm{P}$ value of 0.15 , combined with lower than desired power (0.67) in the face of apparent differences suggest that a type II error could have been committed for this variable and the groups may indeed be different. With a greater number of subjects a statistical difference would likely have seen. There was a significant increase in the perception of pain sensitivity for all muscle sites (rectus femoris, biceps femoris, gastrocnemius) in the land plyometric training group from $0 \mathrm{hr}$ to $48 \mathrm{hr}$ post-training bout at baseline, and when training intensity was increased at week 3 and week $6, p \leq 0.001$; however there was no significant difference in pain sensitivity between the land plyometric and aquatic plyometric training group. Several other factors could have influenced the pain sensitivity results including the individual pain tolerance of each subject and body fat distribution over the belly of the muscle.

Currently, there is no research published that compared the difference in muscle soreness when performing plyometrics on land and in an aquatic setting. However, 
research has shown that aquatic activities are therapeutic and an effective method of strengthening muscles while reducing muscle soreness $(9,54,84,85,88)$. Woods $(88)$ did show that an aquatic low back rehabilitation program resulted in reduced perception of self-report of pain when compared to a land based rehabilitation program. The results of this study support the notion of training adaptations in an aquatic environment. An aquatic setting can provide a safer environment where impact is minimized and buoyancy supports the body. Aquatic training can provide exercise opportunities for people who need to reduce impact on their joints due to fatigue, injury, and disability.

Future studies can be improved by including a more precise measurement of muscle injury and muscle soreness. A biochemical analysis should be used to evaluate creatine kinase and lactate dehydrogenase levels. This will provide a more objective marker for muscle injury and can be used to validate self-report measurement of muscle soreness scales and pain sensitivity.

Numerous avenues can be incorporated into this plyometric training study that could lead to new discoveries in the area of performance enhancement training. One area to examine is the biomechanics of movement on land and in an aquatic environment as it relates to training adaptations for improving muscular strength of the lower extremities. In addition, by using computer digitized analysis of video images, limb angles may be calculated. It would also be interesting to study the effects on aerobic capacity $\left(\mathrm{VO}_{2}\right)$ when performing land and aquatic plyometrics. Max $\mathrm{VO}_{2}$ differences could be studied to determine if plyometric training in an aquatic environment is more metabolically demanding than plyometric training performed on land. With the combination of 
biomechanical analysis and physiological responses (i.e., heart rate and oxygen uptake) a better understanding of the influence of aquatic activities will be achieved.

Another avenue that should be pursued is the study of differences in bone mineral density in land- and aquatic-based plyometric training programs. It would be beneficial to know what effect aquatic activities have on bone mineral density due to the growing number of aquatic exercisers and the increase in osteoporosis in the elderly. It could also be important to investigate the adherence rate and physical activity enjoyment of each training environment in order to enhance adherence. The adherence rates of the land and aquatic plyometric groups were excellent, although one subject from the land plyometric training group did not complete the study due to a medical condition.

It would also be useful to see the results of incorporating weighted vest in plyometric training. This would increase the load, therefore stimulating a greater improvement in power output and force production. This will enable researchers to examine the effects of muscle soreness when performing land- and aquatic-based activities with an added load. Future research should consider investigating the effects of aquatic plyometric training adaptations to the skeletal muscle at the cellular level. This will result in more insights of changes to the muscle fiber/cell in cross-sectional area $\left(\mathrm{CSA}_{\mathrm{f}}\right)$, fiber type characteristics, and hypertrophy. Future studies should also evaluate the effects of body core temperature or thermoregulation, in land- and aquatic-based programs on training adaptations and perception of fatigue.

Future investigations should incorporate a more diverse population to help generalize the results to female athletes. These subjects were physically active collegeage Caucasian females who were currently or previously involved in sports with some 
higher education. This subject pool would better represent the collegiate female athlete if it incorporated more individuals of diverse ethnicity and races. Also, incorporating males into the study would allow the researcher to examine results in both genders. This could lead to future research that examines the effects of plyometric training in male versus females.

This study has shown that an appropriately designed aquatic plyometric training program is effective in enhancing power output, peak torque production, and velocity. This study has also demonstrated that an aquatic plyometric training program places less stress on the body, therefore resulting in a reduced perception of muscle soreness to the rectus femoris, biceps femoris, and gastrocnemius. Therefore, it can be concluded that aquatic plyometric training can provide comparable training gains with a reduced amount of muscle soreness. The results of this study will help to direct coaches, strength specialists, athletic and personal trainers to a more viable training option for conditioning that would enhance performance while reducing muscle soreness.

With additional research, specific guidelines can be established for an effective training program in an aquatic setting. Documentation from future studies, using various types of equipment in many different pool environments will enable the aquatic fitness industry to standardize and grow as we begin to understand the general exercise techniques that are most likely to produce training adaptations. This knowledge will facilitate the development of aquatic-based strength training programs as an alternative to traditional land-based training programs with similar enhancement in performance outcomes and a reduced potential for muscle soreness and possibly muscle injury. 


\section{REFERENCES}

1. Adams, K., J.P. O'Shea, K.L. O'Shea, AND M. Climstein. The effects of six weeks of squat, plyometrics, and squat plyometric training on power production. J. Appl. Sports Sci. Res. 6:36-41. 1992.

2. Anderst, W.J., F. Eksten, AND D.M. Koceja. Effects of plyometric and explosive resistance training on lower body power. Med. Sci. Sports Exerc. 26:S31 \#176. 1994.

3. Arnheim, D.D. Modern principles of athletic training. St. Louis: Times Mirror/Mosby College Publishing, 1985.

4. Asmussen, E., AND F. Bonde-Peterson. Apparent efficiency and storage of elastic energy in human muscles during exercise. Acta Physiol. Scand. 92:537545. 1974.

5. Aura, O., AND J.T. Vitasalo. Biomechanical characteristic of jumping. Int. J. Sports Biomech. 5:89-98. 1989.

6. Beasley, B.L. Aquatic exercise. Sports Med. Dig. 1:1-3. 1988.

7. Bebi, J., A. Cresswell, T. Engel, AND S. Nicoi. Increase in jumping height associated with maximal effort vertical depth jumps. Res. Q. Exerc. Sport 58:1115. 1987.

8. Blattner, S., AND L. Noble. Relative effect of isokinetic and plyometric training on vertical jumping performance. Res. Q. Exerc. Sport 50:583-588. 1979. 
9. Binkley, H.M. Water exercise: effects $\mathrm{n}$ improving muscular strength and endurance in elderly inner city African-American women. Unpublished doctorate's dissertation Temple University, Philadelphia, PA, 1996.

10. Bobbert, M. Drop jumping as a training method for jumping ability. Sports Med. 9:7-22. 1990.

11. Bompa, T.O. Power training for sport: plyometrics for maximum power development. New York: Mosaic Press, 1993.

12. Brown M.E., J.L. Mayhew, AND L.W. Boleach. Effects of plyometric training on vertical jump performance in high school basketball players. J. Sports Med. Phy. Fitn. 26:1-4. 1986.

13. Brzycki, M. Plyometrics: a giant step backwards. Athletic J. 66:22-23. 1986.

14. Campbell, S.M., S. Clarks, E.A. Tindall, M.E. Forehand, AND R.M. Bennett. Clinical characteristics of fibrositis: a "blinded" control study of symptoms and tender points. Arthritis Rheum. 26:817-824. 1983.

15. Case, L. Fitness Aquatics. Champaign, IL: Human Kinetics, 1997.

16. Cavagna, G. Storage and utilization of elastic energy in skeletal muscle. Exerc. Sports Sci. Rev. 5:89-129. 1977.

17. Chu, D. Jumping into plyometrics. Champaign, IL: Human Kinetics, 1998.

18. Clarkson, P.M., K. Nosaka, AND B. Braun. Muscle function after exerciseinduced muscle damage and rapid adaptation. Med. Sci. Sports Exerc. 24:512520. 1992.

19. Clarkson, P.M., AND I. Tremblay. Exercise-induced muscle damage, repair, and adaptations in humans. J. Appl. Physiol. 65:1-6. 1988. 
20. Clarkson, P.M., W.C. Byrnes, K.M. McCormick, L.P. Turcotte, AND J.S. White. Muscle soreness and serum creatine kinase activity following isometric, eccentric, and concentric exercise. Int. J. Sports Med. 7:152-155. 1986.

21. Clutch, D., B. Wilton, M. McGown, AND G.R. Byrce. The effect of depth jumps and weight training on leg strength and vertical jump. Res. Q. Exerc. Sport 54:5-10. 1983.

22. Crowder, V., S.W. Jolly, B. Collins, AND J. Johnson. The effects of plyometric push-up on upper body power. Track Techn. 39:59-67. 1990.

23. Delecluse, C., H. Van Coppenolle, E. Williams, M. Van Leemputte, R. Diels, AND M. Goris. Influence of high-resistance and high-velocity training on sprint performance. Med. Sci. Sports Exerc. 27:1203-1209. 1995.

24. Fatouros, I.G., A.Z. Jamurtas, D. Leonstsini, K. Taxildaris, N. Aggelousis, N. Kostopoulos, AND P. Buckenmeyer. Evaluation of plyometric exercise training, weight training, and their combination on vertical jump performance and leg strength. J. Strength Cond. Res. 14:470-476. 2000.

25. Fields, D.A., M.I. Goran, AND M.A. McCrory. Body-composition assessment via air-displacement plethysmography in adults and children: a review. Am. J. Clin. Nutr. 75:453-467. 2002.

26. Fischer, A.A. Pressure threshold meter: its use for quantification of tender spots. Arch. Phys. Med. Rehab. 67:836-838. 1986.

27. Foster, C., L.L. Hector, K.S. McDonald, AND A.C. Synder. Measurement of Anaerobic Power and Capacity. In. Physiological Assessments of Human Fitness. P.J. Maud and C. Foster, eds. Champaign, IL: Human Kinetics, 1995. pp. 73-85.

28. Fox, E.L., R.W. Bowers, AND M.L. Foss. The physiological basis of physical education and athletics. Dubuque, IA: Brown, 1989. 
29. Friden, J., M. Sjostrom, AND B. Ekblom. A morphological study of delayed muscle soreness. Experientia 37:506-507. 1981.

30. Gatti, C. Water training as a means of maintaining cardiovascular endurance in college males. Unpublished master's thesis Washington University, St. Louis, MO, 1987.

31. Gullick, D.T., I.F. Kimura, M. Silter, A. Paolone, AND J.D. Kelly. Various treatment techniques on signs and symptoms of delayed onset muscle soreness. $J$ Athl. Trng. 31:145-152. 1996.

32. Hakkinen, K., P. Komi, AND M. Allen. Effect of explosive type strength training on isometric force- and relaxation- time, electromyographic and muscle fibre characteristics of leg extensors muscles. Acta Physiol. Scand. 125:587-600. 1985.

33. Hamlin, M.J., AND B. Quigley. Quadriceps concentric and eccentric exercise: differences in muscle strength, fatigue and EMG activity in eccentricallyexercised sore and non-sore muscles. J. Sci. Med. Sport 4:104-115. 2001.

34. Hewett, T.E., J.V. Riccobene, AND T.N. Lindenfeld. A prospective study of the effects of neuromuscular training on the incidence of knee injury in female athletes [abstract]. In: Book of abstracts and outlines for the $24^{\text {th }}$ Annual Meeting of the American Orthopaedic Society for Sports Medicine; July 12-15, 1998; Vancouver, British Columbia, Canada. Abstract 346.

35. Hewett, T.E., A.L. Stroupe, T.A. Nance, AND F.R. Noyes. Plyometric training in female athletes. Am. J. Sports Med. 24:765-773. 1996.

36. High, M.D., E.T. Howley, AND B.D. Franks. The effects of static stretching and warm-up on prevention of delayed-onset muscle soreness. Res. Q. 60:357361. 1989.

37. Hiserman, J. Plyometrics for jumpers. American Athletics 5:33-38. 1993. 
38. Hogeweg, J.A., M.J. Langereis, A.T. Bernards, J.A. Faber, AND P.J. Helders. Algometry. Scand. J. Rehab. Med. 24:99-103. 1992.

39. Holcomb, W.R., J.E. Lander, R.M. Ryttland, AND G.D. Wilson. The effectiveness of modified plyometric program on power and the vertical jump. $J$. Strength Cond. Res. 10:89-92. 1996.

40. Hough, T. Ergographic studies in muscular soreness. Am. J. Physiol. 7:72-92. 1902.

41. Horrigan, J., AND D. Shaw. Plyometrics: think before you leap. Track field $Q$. Rev. 89:41-43. 1989.

42. Hutchinson, M.R., AND M.L. Ireland. Knee injuries in female athletes. Sports Med. 19:288-302. 1995.

43. Jamurtas, A.Z., I.G. Fatouros, P. Buckenmeyer, E. Kokkinisis, K. Taxildaris, A. Kambas, AND G. Kyriazis. Effects of plyometric exercise on muscle soreness and plasma creatine kinase levels and its comparison with eccentric and concentric exercise. J. Strength Cond. Res. 14:68-74. 2000.

44. Johansson, P., L. Lindstrom, G. Sundelin, AND B. Lindstrom. The effects of preexercise stretching on muscular soreness, tenderness and force loss following heavy eccentric exercise. Scand. J. Med. Sci. Sports 9:219-225. 1999.

45. Kaminski, M., AND R. Boal. An effect of ascorbic acid on delayed-onset muscle soreness. Pain 50:317-321. 1992.

46. Katz, B. The relation between force and speed in muscle contraction. J. Physiol. 96:45-64. 1939.

47. Keele, K.D. Pain-sensitivity tests: pressure algometer. Lancet 1:636-639. 1954.

48. Klavora, P. Vertical-jump tests: a critical review. Strength Cond. J. 22:70-75. 2000. 
49. Kraemer, W.J., AND A.C. Fry. Strength testing: development and evaluation of methodology. In: Physiological Assessment of Human Fitness. P.J. Maud and C. Foster, eds. Champaign, IL: Human Kinetics, 1995. pp. 115-138.

50. Kraemer, W.J., AND R.U. Newton. Training for improved vertical jump. Gatorade Sports Sci. Inst. Rep. - Sports Sci. Exchange \#53(7). 1994.

51. Lansbury, J. Methods for evaluating rheumatoid arthritis. In: Rheumatology. J.L. Hollander, ed. Philadelphia, PA: Lea \& Febiger, 1966. pp. 269-291.

52. Levene, J.A., B.A. Hart, R.H. Seeds, AND G.A. Fuhrman. Reliability of reciprocal isokinetic testing of the knee extensors and flexors. J. Orthop. Sports Phys. Ther. 14:121-127. 1991.

53. Lundin, P., AND W. Berg. A review of plyometric training. Nat. Strength Cond. Assoc. J. 13:22-30. 1991.

54. Martin, M.M. Strength gains through aquatic exercise. Unpublished master's thesis Springfield College, Springfield, MA, 1992.

55. McCrory, M.A., T.D. Gomez, E.M. Bernauer, AND P.A. Mole. Evaluation of a new air displacement plethysmograph for measuring human body composition. Med. Sci. Sports. Exerc. 27:1686-1691. 1995.

56. Meydani, M., W.J. Evans, AND G. Handelman. Protective effect of vitamin E on exercise-induced oxidative damage in young and older adults. Am. J. Physiol. 264:999-998. 1993.

57. Miller, J.L., M.D. Heinrich, AND R. Baker. A look at Title IX and women's participation in sport. Physical educator 57:8-13. 2000.

58. Moldofsky, H., P. Scarisbrick, R. England, AND H. Smythe. Musculoskeletal symptoms and non-REM sleep disturbances in patients with "fibrositis syndrome" and healthy subjects. Psychosom Med. 37:341-351. 1975. 
59. Moldofsky, H., AND W.J. Chester. Pain and mood patterns in patients with rheumatoid arthritis: prospective study. Psychosom Med. 32:309-318. 1970.

60. Newman, D., D. Jones, AND R. Edwards. Plasma creatine kinase changes after eccentric and concentric contractions. Muscle Nerve 6:59-63. 1986.

61. Nigg, B.M., AND W. Herzog. Biomechanics of the musculo-skeletal systems. New York: John Wiley, 1994.

62. Osternig, L.R. Isokinetic dynamometry: implications for muscle testing and rehabilitation. In. Exercise and Sport Science. K.B. Pandolf, ed. New York, NY: MacMillan Publishing, 1986. pp. 45-104.

63. Parcells, R. The effect of depth jumping and weight training on vertical jump. Unpublished master's thesis Ithaca College. 1977.

64. Paffenbarger, R.S., A. Wing, and R. Hyde. Physical activity as an index of heart attack risk in college alumni. Am. J. Epidemiol. 109:161-17. 1975.

65. Politino, V., M. McCormick, AND A. Jefferys. Lifetime Physical Fitness. Dubuque, IA: Kendell/Hunt, 1995.

66. Potteiger, J.A., R.H. Lockwood, M.D. Haub, B.A. Dolezal, K.S. Alumzaini, J.M. Schroeder, AND C.J. Zebas. Muscle power and fiber characteristic following 8 weeks of plyometric training. J. Strength Cond. Res. 13:275-279. 1999.

67. Prapavessis, H., AND P.J. McNair. Effects of instruction in jumping technique and experience jumping on ground reaction forces. J. Orthop. Sports Phys. Ther. 29:353-356. 1999.

68. Radcliffe, J., AND R. Farentinos. Plyometrics - explosive power training. Champaign, IL: Human Kinetics, 1985. 
69. Rimmer E., AND G. Sleivert. Effects of a plyometric intervention program on sprint performance. J. Strength Cond. Res. 14:295-301. 2000.

70. Rocan, S. Aqua fitness - an overview. Fit. Leader 2:25-29. 1984.

71. Ruoti, R.G., J.T. Troup, AND R.A. Berger. The effects of non-swimming water exercises on older adults. J. Orthop. Sports Phys. Ther. 19:140-145. 1994.

72. Safrit, M. Complete guide to youth fitness testing. Champaign, IL: Human Kinetics, 1995.

73. Sala, D., AND D. MacDougall. Specificity in strength training: a review for the coach and athlete. Can. J. Appl. Sport Sci. 6:87-92. 1981.

74. Saxton, J.M., P.M. Clarkson, R. James, M. Miles, M. Westerfer, S. Clark, A.E. Donnelley. Neuromuscular dysfunction following eccentric exercise. Med. Sci. Sports Exerc. 27:1185-1193. 1995.

75. Schmidtbleicher, D. Presentation given to the National Strength and Conditioning Association. San Diego, CA. 1990.

76. Schwane, J.A., AND R.B. Armstrong. Effect of training and skeletal muscle injury from downhill running in rats. J. Appl. Physiol. 55:969-975. 1983.

77. See, J. Exercising options: kickboxing and personal training have hit the water and the water doesn't hit back. Aquatic international 13:34-39. 2001.

78. Sova, R. Aquatics. Boston, MA: Jones and Bartlett Publishers, Inc., 1992.

79. Suomi, R., AND S. Lindauer. Effectiveness of arthritis foundation aquatic program on strength and range of motion in women with arthritis. J. Aging Phys. Activity 5:341-351. 1997. 
80. Thein, J.M. AND L.T. Brody. Aquatic-based rehabilitation and training for the elite athlete. J. Orthop. Sports Phys. Ther. 27:32-41. 1998.

81. Thomas, D.W. Plyometrics-more than a stretch reflex. Nat. Strength Cond. Assoc. J. 10:49-51. 1988.

82. Wagner, D.R., AND V.H. Heyward. Techniques of body composition assessments: a review of laboratory and field methods. Res. Q. Exerc. Sport 70:135-149. 1999.

83. Wathen, D. Literature review: explosive/plyometric exercises. Natl. Strength Cond. Assoc. J. 15:17-19. 1993.

84. Weinstein, L.B. Benefits of aquatic activity. J. of Gernotological Nursing 12:611. 1986.

85. White, T., AND B.S. Smith. The efficacy of aquatic exercise in increasing strength. Sports Med. Training Rehab. 9:51-59. 1999.

86. Wilson, G.J., R.U. Newton, A.J. Murphy, AND B.J. Humphries. The optimal training load for the development of dynamic athletic performance. Med. Sci. Sports Exerc. 25:1279-1286. 1993.

87. Wilk, K.E., C. Arrigo, J.R. Andrews, AND W.G. Clancy. Rehabilitation after anterior cruciate ligament reconstruction in the female athlete. J. Athletic Training 34:177-193. 1999.

88. Woods, D.A. Rehabilitation aquatics for low back injury: functional gains or pain reduction? Clinical Kinesiology 43:96-103. 1989.

89. Yamagata, S., M. Ishikawa, M. Saijo, AND S. Fukushima. Diagnostic reevaluation of electric skin resistance, skin temperature and deeper tenderness in patients with abdominal pain. Tohoku J. Exp. Med. 118:183-189. 1976. 
90. Yessis, M. Speed/explosiveness with plyometrics. Scholastic Coach 60:32-34. 1991. 
APPENDIX A

INSTITUTIONAL REVIEW BOARD APPROVAL 
BIOMEDICAL SCIENCES

INSTITUTIONAL REVIEW BOARD

RESEARCH INVOLVING HUMAN SUBJECTS

THE OHIO STATE UNIVERSITY
$\mathrm{X}$ Original Review

Continuing Review

Amendment

Reactivation

\section{ACTION OF THE REVIEW BOARD}

With regard to the employment of human subjects in the proposed research:

01H0433 EFFECTS OF LAND VERSUS AQUATIC PLYOMETRICS OF POWER, FORCE, VELOCITY, AND MUSCLE SORENESS, Janet

Buckworth, Leah E. Robinson, Steven T. Devor, Physical Activity and Education Services

THE BIOMEDICAL SCIENCES REVIEW BOARD HAS TAKEN THE FOLLOWING ACTION:

APPROVED
$\mathbf{X}$ APPROVED WITH STIPULATION(S)*
$\begin{aligned} & \text { *Stipulation(s) stated by the IRB have been met by the } \\ & \text { investigator, and therefore, the protocol is APPROVED. }\end{aligned}$

Approval for proposed research includes all materials submitted by the investigator unless otherwise noted.

It is the responsibility of the principal investigator to retain a copy of each signed consent form for at least three (3) years beyond the termination of the subject's participation in the proposed activity. Should the principal investigator leave the University, signed consent forms are to be transferred to the Human Subjects Review Board for the required retention period. This application has been approved for a period of not more than one year. You are reminded that you must promptly report any problems to the Review Board, and that no procedural changes may be made without prior review and approval. You are also reminded that the identity of the research participants must be kept confidential.

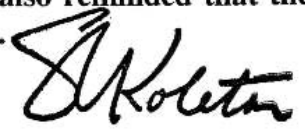

Date: December 17,2001 Signed:

Chairperson

HS-025H (Rev. 1/00) 
Original Review Continuing Review

X Amendment

Reactivation

\section{ACTION OF THE REVIEW BOARD}

With regard to the employment of human subjects in the proposed research:

01H0433 EFFECTS OF LAND VERSUS AQUATIC PLYOMETRICS OF POWER, FORCE, VELOCITY, AND MUSCLE SORENESS, Janet Buckworth, Leah E. Robinson, Steven T. Devor, PAES

Request for change(s) to the protocol dated February 14, 2002, addition of Pomerene Hall gymnasium and aquatic facilities, was APPROVED BY EXPEDITED REVIEW.

THE BIOMEDICAL SCIENCES REVIEW BOARD HAS TAKEN THE FOLLOWING ACTION:

$\underline{X}$ APPROVED

APPROVED WITH STIPULATION $(S)^{*}$

*Stipulation(s) stated by the IRB have been met by the investigator, and therefore, the protocol is APPROVED.
DISAPPROVED

WAIVER OF WRITTEN

CONSENT GRANTED

Approval for proposed research includes all materials submitted by the investigator unless otherwise noted.

It is the responsibility of the principal investigator to retain a copy of each signed consent form for at least three (3) years beyond the termination of the subject's participation in the proposed activity. Should the principal investigator leave the University, signed consent forms are to be transferred to the Human Subjects Review Board for the required retention period. This application has been approved for a period of not more than one year. You are reminded that you must promptly report any problems to the Review Board, and that no procedural changes may be made without prior review and approval. You are also reminded that the identity of the research participants must be kept confidential.

Date: February 19,2002 Signed:

HS-025H (Rev, 1/00)

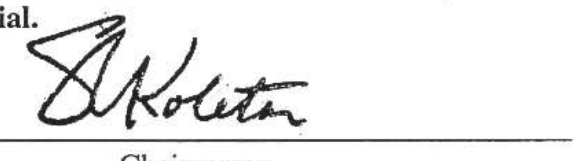

Chairperson 


\section{APPENDIX B}

INFORMED CONSENT 


\section{CONSENT TO INVESTIGATIONAL TREATMENT OR PROCEDURE}

I, , hereby authorize or direct Janet Buckworth, Ph.D., associates or assistants of his/her choosing, to perform the following treatment or procedure (describe in general terms), which is to evaluate and compare the effects of eight (8) weeks of strength and jump training (plyometric training) on land and in an aquatic setting on power, force, velocity, and muscle soreness. The training assessments and sessions will occur in both Larkins Hall (377 West 17th Avenue) and Pomerene Hall (1760 Neil Avenue) on the campus of The Ohio State University. My participation in the study will include the completion of three (3) questionaires, four (4) history information forms, eight (8) weeks of strength and jump training \{three (3) days per wk for 50 minutes\}, and assessments of power, force, and velocity using vertical jump, strength testing, and a timed 40meter run (about 131 feet), respectively. Muscle soreness of the quadricep muscles will be analyzed through the response of direct muscle manipulation, an establish ordinal scale, and the Short-Form McGill Pain Questionaire. I will be matched based on pretest vertical jump and randomly assigned to a land or an aquatic plyometric training program. I will be asked to refrain from taking more than the Recommend Daily Allowances (RDA) of antioxidants (i.e., vitamin A, vitamin E, and vitamin C), discontinue the use of ergogenic (supplement) aids (i.e., anabolic streoids and creatine supplementation) and the participation in other forms of strength and aerobic training during the course of this study.

upon

(myself or name of subject)

The experimental (research) portion of the treatment or procedure is:

1. To participate in an eight (8) week strength and jump training program on land or in an aquatic setting.

2. To participate in baseline (pre-training), mid-training, and post-training measurements, which will allow the examination of changes in the performance outcome variables (power, force, velocity) and in muscle soreness.

This is done as part of an investigation entitled:

The Effects of Land versus Aquatic Plyometric Training on Power, Force, Velocity, and Muscle Soreness

\section{Purpose of the procedure or treatment:}

The purpose of this study is to evaluate and compare eight (8) weeks of land and aquatic plyometric training on females' power, force, velocity, and muscle soreness.

2. Possible appropriate alternative procedure or treatment (not to participate in the study is always an option):

The alternative to performing the training program is to not participate in the study.

\section{Discomforts and risks reasonably to be expected:}

Because I will be participating in a training program with unusual exertion, muscle soreness may be present for the first 24-72 hours. The soreness will subside as I become adapted to the exertion associated with strength 
training. I am aware that I may experience discomfort if I am selected to particpate in the aquatic training group, however I will be provided water shoes to aid in the prevention of wear and tear on my feet.

\section{Possible benefits for subjects/society:}

I will gain an increase in my lower body strength, which can add more defination to my thigh muscles. I will improve my vertical jump (power), strength (force produced), and speed (velocity). I will also gain the experience of what it is like to be a subject in a exercise training study. I will know that I am participating in a study that is being performed on a topic that has never been researched before. This study will contribute to what we know about sports performance enhancement, particularly regarding female athletes.

I am also aware that I have the opportunity to win cash money based on my participation in this study. The first random cash drawing is for those who attend all of the training and testing sessions during the $1^{\text {st }}$ week of the training protocol. Twenty $\$ 10.00$ prizes will be distributed. The second cash drawing is for individuals who attend at least $85 \%$ ( 20 sessions) of the training and all of the testing sessions. Eight $\$ 25.00$ prizes will be distributed. An ice cream party will be provided for all of the subjects in the study the week after the testing is completed.

\section{Anticipated duration of subject's participation (including number of visits):}

I will visit a total of thirty-four (34) times during this study. Each visit will take approximately 50 minutes. The first two (2) visits will include the completion of the four (4) background history information forms and baseline measurements of power, forces, and velocity. The measurements of level of physical activity, height, body mass, percentage body fat, and leg length measurements will also be conducted. The training protocol will begin April 8,2002 (week 2 of a 10 week quarter). The first week of training will consist of a series of training sessions (bouts) followed by testing to assess muscle soreness for the first $48-72$ hours of training. This will produce baseline and pre-training measurements that will allow the examination of improvements (gains) and/or changes in the performance outcome variables (Power, Force, and Velocity). There will be a total of twenty-four (24) training sessions over a period of eight (8) weeks. Mid-Training assessments will be collected during the 5 th week of Spring Quarter (May 6th-10th) on Tuesday and Thursday. Plyometric training sessions will continue to occur on Monday, Wednesday, and Friday. Post-Training assessments will be collected during the 10th week of Spring Quarter (June 3rd-7th).

I hereby acknowledge that has provided information about the procedure described above, about my rights as a subject, and he/she answered all questions to my satisfaction. I understand that I may contact him/her at Phone No. 614-292-0757 should I have additional questions. $\mathrm{He} / \mathrm{She}$ has explained the risks described above and I understand them; he/she has also offered to explain all possible risks or complications.

I understand that, where appropriate, the U.S. Food and Drug Administration may inspect records pertaining to this study. I understand further that records obtained during my participation in this study that may contain my name or other personal identifiers may be made available to the sponsor of this study. Beyond this, I understand that my participation will remain confidential.

I understand that $I$ am free to withdraw my consent and participation in this project at any time after notifying the project director without prejudicing future care. No guarantee has been given to me concerning this treatment or precedure. 
i understand in signing this form that, beyond giving consent, I am not waiving any legal rights that I might otherwise have, and $I$ am not releasing the investigator, the sponsor, the institution, or its agents from any legal liability for damages that they might otherwise have.

In the event of injury resulting from participation in this study, I also understand that immediate medical treatment is available at University Hospitals of The Ohio State University and that the costs of such treatment will be at my expense; financial compensation beyond that required by law is not available. Questions about this should be directed to the Office of Research Risks at (614) 688-4792.

I have read and fully understand the consent form. I sign it freely and voluntarily. A copy has been given to me.

Date: $-\frac{10}{\text { AM }}$

PM Signed

(Subject)

Witness (es)

if required

(Person Authorized to Consent for Subject if Required)

I certify that $I$ have personally completed all blanks in this form and explained them to the subject or his/her representative before requesting the subject or his/her representative to sign it.

Date:

Signed

(Signature of Project Director or his/her Authorized Representative)

HS-028A (Rev. 7/93) 
APPENDIX C

PAFFENBARGER PHYSICAL ACTIVITY QUESTIONNAIRE 


\section{Paffenbarger Physical Activity Questionnairc}

1. How many city blocks or their equivalent do you normally walk each day? blocks/day

$$
\text { (Let } 12 \text { blocks }=1 \text { milc) }
$$

2. What is your usual pace of walking? (Please check one.)
a. Casual or strolling (less than $2 \mathrm{mph}$ )
b. Average or normal (2 to $3 \mathrm{mph}$ )
c. ___ Fairly brisk ( 3 to $4 \mathrm{mph}$ )
d. Brisk or striding ( $4 \mathrm{mph}$ or faster)

3. How many flights or stairs to you climb up each day? _ flights/day (Let 1 flight $=10$ steps.)

4. List any sports or recreation you have actively participated in during the past year.

Please remember seasonal sports or events.

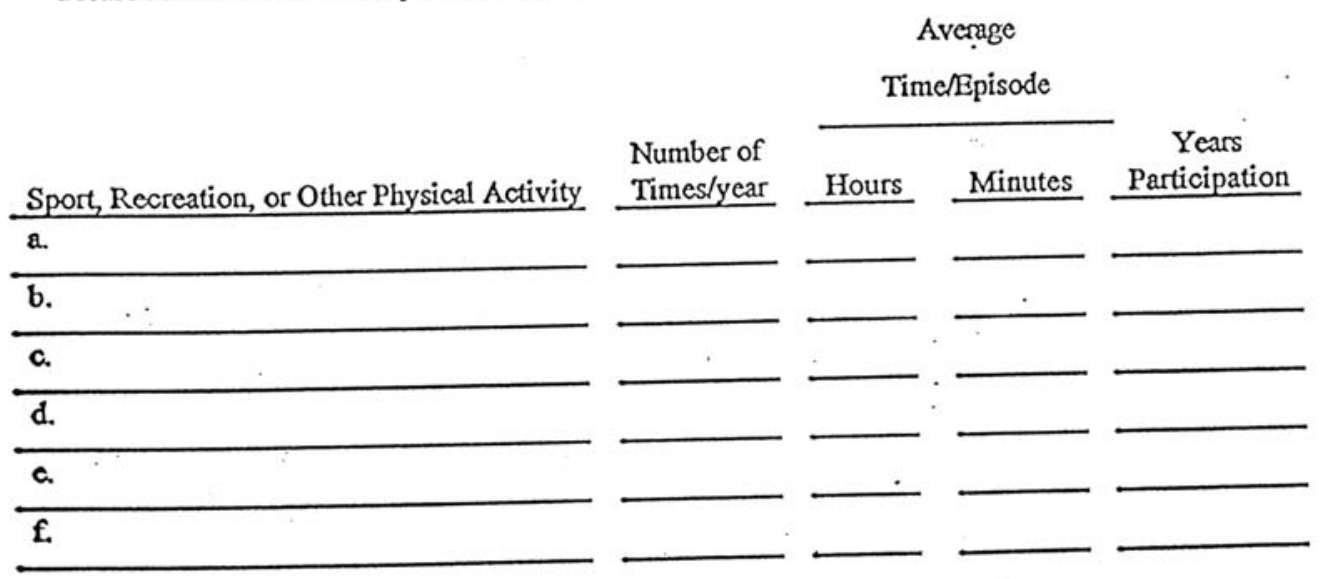

5. Which of these statements best expresses your view? (Please check one.)

a. I take enough exercise to keep healthy. b. _ I ought to take more exercise. c. __ Don't know

6. At least once a week, do you engage in regular activity akin to brisk walking, jogging, bicycling, swimming, etc. long enough to work up a sweat, get your heart thumping, or get out of breath? No Why not? ___ Yes How many times per weck? - Activity:

7. When you are exercising in your usual fashion, how would you rate your lèvel of exertion (degree of effort)? (Please circle one number.)

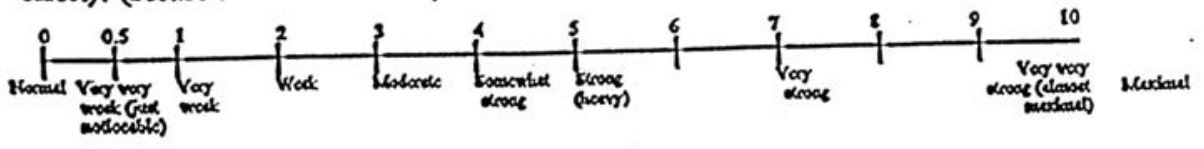


8. On a usual weekday and a weekend day, how much time do you spend on the following activities? Total for cach day should add to 24 hours.

\begin{tabular}{|c|c|c|}
\hline & $\begin{array}{l}\text { Usual Wockday } \\
\text { Hours/Day }\end{array}$ & $\begin{array}{l}\text { Usual Weckend Day } \\
\text { HoursDay }\end{array}$ \\
\hline $\begin{array}{l}\text { a. Vigorous activity (digging in the garden, strenuous } \\
\text { sports, jogging, aerobic dancing, sustained } \\
\text { swimming, brisk walking, heavy carpentry, } \\
\text { bicycling on hills, etc.) }\end{array}$ & & \\
\hline $\begin{array}{l}\text { b. Moderatc activity (housework, light sports, regular } \\
\text { walking, golf, yard work, lawn mowing, painting, } \\
\text { repairing, light carpentry, ballroom dancing, } \\
\text { bicycling on level ground, etc.) }\end{array}$ & & \\
\hline $\begin{array}{l}\text { c. Light activity (office work, driving car, strolling, } \\
\text { personal care, standing with little motion, etc.) }\end{array}$ & & \\
\hline $\begin{array}{l}\text { d. Sitting activity (eating, reading, desk work, } \\
\text { watching TV, listening to radio, etc.) }\end{array}$ & - & \\
\hline c. Slecping or reclining & & \\
\hline
\end{tabular}


APPENDIX D

DEMOGRAPHIC FORM 


\section{DEMOGRAPHIC FORM}

Name:

Local Address:

Permanent Address:

Phone Number: E-Mail:

Date of Birth:

How would you describe your ethnicity? (Please check all that apply)

$\square \quad$ White (Non-Hispanic)

$\square \quad$ Asian or Pacific Islander

$\square \quad$ American Indian or Alaskan

$\square \quad$ Other:

Do you check your e-mail regularly: $\quad \square$ Yes $\quad \square$ No

ID \#

$* * * * * * * * * * * * * * * * * * * * * * * * * * * * * * * * * * * * * * * * * * * * * * * * * * * * * * * * * * * * * * * *$

Sport \& Exercise History

\begin{tabular}{|l|l|l|}
\hline Sports & $\begin{array}{l}\text { Team (Yes/No); if yes, please specify recreational, } \\
\text { interscholastic, and/or collegiate }\end{array}$ & Years \\
\hline & & \\
\hline & & \\
\hline & & \\
\hline & & \\
\hline & & \\
\hline
\end{tabular}

How long have you been exercising regularly that raises heart rate, breath heavily, and sweat? (Regular is defined as exercising at least 3 times a week for at least 30 minutes at a time)

Years

Months

Weeks

OSU Office of Research Risk Protection

Protocol \#01H0433 
If yes, please list how many times per week and for how long you perform each of the following:

$\square$ Swimming

Bike Riding (Stationary)

$\square$ Bike Riding (Outside)

$\square$ Weight Training

$\square$ Cross Training

Other times per week times per week times per week times per week times per week times per week total minutes each week total minutes each week total minutes each week total minutes each week total minutes each week total minutes each week

Have you ever participated in any type of water activities (i.e., water aerobics, water walking, swimming, etc.) and please specify

Do you have a fear of water?

Yes No

If yes, will the chance of you being randomly selected to the aquatic training group effect your participation in this research. You will be performing plyometric activities in 4 to $4 \frac{1}{2}$ feet of water.

Yes $\square$ No 


\section{APPENDIX E}

GENERAL MEDICAL HISTORY FORM 
ID \#:

\section{GENERAL MEDICAL HISTORY FORM}

Please answer the following questions concerning your history of injuries or illness.

\section{Head}

1. Do you experience headaches?

Yes

No

If yes, how frequently do they occur? Per week per month

Where are they located? Front Back Sinus Temple

(check all that apply) Right Side Left side Behind the eyes

Do you take any medication or medicine to relieve them?

Yes

No

If yes, what do you use?

2. Have you ever had a head or neck injury?

Yes

No

If yes, please specify date?

If yes, did you experience a loss of consciousness?

Yes

No

If yes, how long were you unconscious? hrs. $\min$.

Were you under the care of a physician?

Yes No

Were you hospitalized?

Yes

No

Did you experience any paralysis?

Yes

No

If yes, please specify location:

Did you experience any numbness, tingling, or weakness of extremities?

Yes

No

If yes, please specify location:

How long before you resumed normal activity?

3. Do you experience blackouts? days

If yes, how frequently do they occur? Yes No

If yes, please specify the date(s)

If yes, what do you do when they occur?

OSU Office of Research Risks Protection

Protocol \#01H0433 


\section{Heart}

1. Do you have any heart problems?

Yes

No

If yes, please specify

2. Do you have a family history of heart disease?

Yes

No

If yes, please specify

3. Have you ever been told that a member of your family died suddenly or had a heart attack at young age (younger than 50 yrs of age)?

Yes

No

If yes, please specify

\section{General}

1. Do you have asthma?

Yes No

If yes, do you take any medication?

Yes

No

If yes, what medication?

2. Has your weight change more than $8-10 \mathrm{lbs}$ in the last three months?

Yes

No

If yes, were you dieting or cardiovascular training?

Please explain/specify:

3. Are you currently taking any medications, ergogenic aids, antioxidants (vitamins), and/or supplements?

Yes

No

If yes, please specify (e.g. vitamins, aspirins, antibodies, hormones, etc)

If yes, will you be willing to discontinue the usage of any antioxidants (vitamins) for the duration of this study. Yes No

4. Do you smoke or use any tobacco products regularly?

Yes

No

5. Are you now or do you think that you maybe pregnant?

Yes

No

Please approximate the day of you last menstruation cycle

Will you agree to notify me if you become pregnant during the duration of this study

OSU Office of Research Risks Protection

$$
\text { Yes No }
$$

Protocol \#01H0433 


\section{APPENDIX F}

MUSCULOSKELETAL MEDICAL HISTORY FORM 
ID \#:

\section{MUSCULOSKELETAL MEDICAL HISTORY FORM}

Please answer the following questions concerning your history of muscle or skeletal injuries

1. Have you ever had a back injury?

Yes No

If yes, please specify location and date(s) Upper Middle Lower

2. Have you ever been diagnosed with scoliosis (i.e. curve of the spine)?

\begin{tabular}{lcc} 
If yes, was the scoliosis corrected? & Yes & No \\
Have you ever been told you have a curve in your back? & Yes & No \\
$\quad$ If yes, please explain. & Yes & No \\
& & \\
\hline
\end{tabular}

3. Do you have any leg length discrepancies (i.e. is one leg longer)? Yes

No If yes, please explain.

4. Have you every been told you have patello-femoral syndrome (PFS)

\begin{tabular}{lcc} 
5. Do you have plantar fasciitis (i.e. heel spur syndrome) & Yes & No \\
$\begin{array}{l}\text { 6. Do you wear orthotics? } \\
\quad \text { If yes, please explain. }\end{array}$ & Yes & No \\
\hline
\end{tabular}

OSU Office of Research Risks Protection

Protocol \#01H0433 
7. Have you ever had a dislocation?

If yes, please give location, explanation, and date(s).

Location:

Explanation \& date(s):

8. Have you ever broken a bone?

Yes

No

If yes, please give location, explanation, and date(s).

Location:

Explanation \& date(s):

9. Have you ever sprained or torn any joints?

Yes

No

If yes, please give location, explanation, and date(s).

Location:

Neck

Shoulder

Elbow

Wrist

Hip

Back

Knee

ACL

PCL

MCL

LCL

Ankle

Medial

Lateral

Explanation \& date(s):

10. Do you hear any "popping or clicking" in any joints?

Yes

No If yes, please explain.

11. Do any of your joints feel as if it is slipping?

Yes

No If yes, please explain.

OSU Office of Research Risks Protection

Protocol \#01H0433 
12. Do you have a pin, plate, screw, or anything metal in your body?

Yes

No

If yes, please explain.

13. Have you ever had surgery?

Yes

No

If yes, please specify date(s) and reason(s).

Reason(s) \& date(s) for surgery.

Did surgery correct the problem?

Yes

No

If no, please explain.

OSU Office of Research Risks Protection

Protocol \#01H0433 
APPENDIX G

PLYOMETRIC TRAINING STUDY TIMELINE 


\section{PLYOMETRIC TRAINING STUDY TIMELINE}

Week $1 \quad$ - Performance variables pre-training assessment (MWF)

Week 2 - Training: Week 1 (MWF)

- Muscle soreness baseline assessment (0, 48, $96 \mathrm{hr}$ after first training bout)

Week 3 - Training: Week 2 (MWF)

Week $4 \quad$ - Training: Week 3 (MWF)

- Increase in training volume and intensity

- Second muscle soreness assessment (0, 48, $96 \mathrm{hr}$ after first training bout)

Week 5 - $\quad$ Training: Week 4 (MWF)

- Performance variables mid-training assessment

- $\quad(\mathrm{T} \mathrm{Th})$

Week $6 \quad$ - Training: Week 5 (MWF)

Week $7 \quad$ - Training: Week $6(\mathrm{MWF})$

- Increase in training volume and intensity

- $\quad$ Third muscle soreness assessment (0, 48, $96 \mathrm{hr}$ after first training bout)

Week $8 \quad$ - $\quad$ Training: Week 7 (MWF)

Week 9 - Training: Week 8 (MWF)

Week 10 - $\quad$ Performance variables post-training assessment 


\section{APPENDIX H}

WARM-UP (STRETCHING) PROGRAM 


\section{WARM-UP (STRETCHING) PROGRAM}

The warm-up (stretching) program has been revised from Ruoti et al., (71). A description of each exercise and duration is below.

1. Lateral Lift -60 seconds

Stand erect with you body facing 90 degrees away from the pool or gym wall. Stabilize your body by extending one arm to grip the pool or gym wall.

Lift you outer leg as high as possible laterally (to the side) but not above the level of you hip.

Lower the left leg downward and across the front of the supporting leg as far as possible

Repeat the lifting motion. This time lower the leg downward and across the back of the supporting leg.

Repeat with equal force in both directions. Reverse body position and repeat exercise with other leg.

2. $\quad$ Left-Right-Left -60 seconds

Stabilize your upper body to the pool or gym wall by standing with your back against the wall. Raise one leg in front to a 45 degree position with your foot pointed. Keep the supporting leg straight with the foot flat on the pool of gym floor.

While keeping you leg straight, switch between the right and left leg.

3. $\quad$ Leg Stretch -60 seconds

Face the side of the pool or gym wall approximately a leg's length distance. Lift one leg forward allowing it to rest on the wall.

Slowly straighten your leg. Hold this stretch position for 6-8 seconds.

Repeat with the other leg. 
4. Quadriceps Stretch -60 seconds

Face the pool or gym wall with both feet flat on the floor and extend your arms forward to grip the wall.

Initiate a backward kicking (lifting) motion, allowing you lower leg to bend at the knee and swing upwards. Repeat.

Repeat exercise with other leg.

5. Hamstring stretch -60 seconds

Face the wall of the pool or gym and place you hands against the wall.

Flex at the hips with the legs straight together.

Begin to walk down the wall slowly with you hands.

Once you reach your maximal stretch hold for 30 seconds.

6. $\quad$ Hip Dip -60 seconds

Stand and position your body perpendicular to the wall. Extend you inside are (straight) and take a grip on the wall. Now take a short side step away from the wall.

Keep you gripping arm straight as you dip your hip as close as possible toward the wall.

Now extend your hip as far away from the wall as possible and arch your other arm over your head. Hold this position for ten seconds.

Alternate these two movements in a continued rhythmic motion. Turn around and repeat the exercise in the other direction.

7. $\quad$ Arm Cross Over -60 seconds

Extend straight arms in a crossed position in front of the body. Your hands are in a vertical, 'thumbs-up' position.

In a sweeping motion, swing your arms behind your as far as possible.

In a sweeping forward motion, thrust the arms to the starting position. 
8. $\quad$ Side Wall Push Off -60 seconds

Stand erect while facing and pressing the wall. Your toes should be touching the wall. Reach up and grip the wall with both hands.

First, keep you body straight and push yourself away from the all.

Next, pull yourself to the starting position. Push and pull vigorously with equal force.

9. $\quad$ Calf stretch -60 seconds

Face a wall with you hands resting again the wall.

Extend one leg behind, keeping the heel on the floor.

Bend the knee of the opposite leg. Hold for 30 seconds.

Repeat with other leg.

Repeat the full stretch with the back knee slightly bent. 
APPENDIX I

PLYOMETRIC TRAINING PROTOCOL 


\section{PLYOMETRIC TRAINING PROTOCOL}

The plyometric training protocol was adapted and revised from Chu (17) and Radcliffe \& Farentinos (68). A description and illustration of each drill, the training volume and intensity, along with the application of the each drill to a specific sport is included.

Two Foot Ankle Hops- serves as an overall body warm-up to get accustomed to ground impact forces.

Applications: to all sports (especially baseball, tennis, and volleyball)

Start: Stand with feet shoulder-width apart and the body in a vertical position.

Action: Using only the ankles for momentum, hop continuously in one place.

Extend the ankles to the maximum range on each vertical jump.

TIP

- Use your arms to help develop momentum.

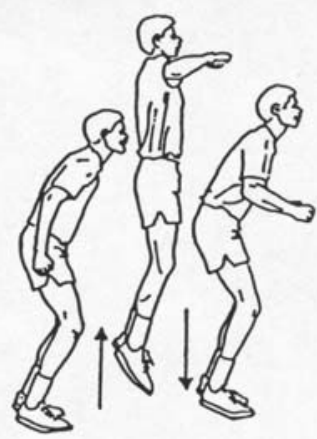

Heel Kick Jumps - performed as a series of rapid explosive jumps that exercise the hip and leg flexors, gastrocnemius, gluteus, and quadriceps.

Applications: all sports (especially downhill skiing, figure skating, and gymnastic)

Start: Assume a relaxed position with feet shoulder-width apart and the body in a straight vertical position with arms by your side.

Actions: Keeping the knees pointed down (still in line with body), jump and kick the buttocks with the heels. Repeat the jump immediately. This is a quick-stepping action from the knees and lower legs. Swings your arms up as you jump.

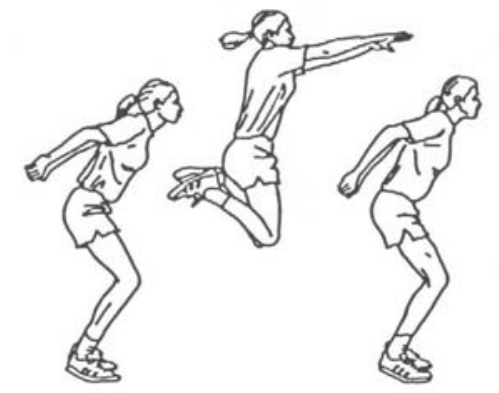


Power Skipping (High Knees) - with full flexion of the leg, is an excellent drill for working the striding muscles (gluteus, gastrocnemius, quadriceps, hamstrings, and hip flexors). Lower back, abdominals, and shoulder girdle are also involved.

Applications: all sports, especially for running, high jumpers, and crosscountry/downhill skiing

$\underline{\text { Start: }}$ Assume a relaxed standing position with one leg slightly forward. Stand comfortably.

Actions: Driving off with the back leg, initiate a short skipping step, then with the opposite leg thrust the knee up to chest level (upon landing, repeat the action with the opposite leg). The pattern of right-right step-left-left step-etc (skipping motion). Goal is to obtain as much height and explosive power as possible after each short step.

Remember to drive the knee up hard and fast to generate maximal lift. Also, us the arms to initiate the life after each skip. Concentrate on "hang-time" of the body and minimize the time the feet are in contact with the ground.

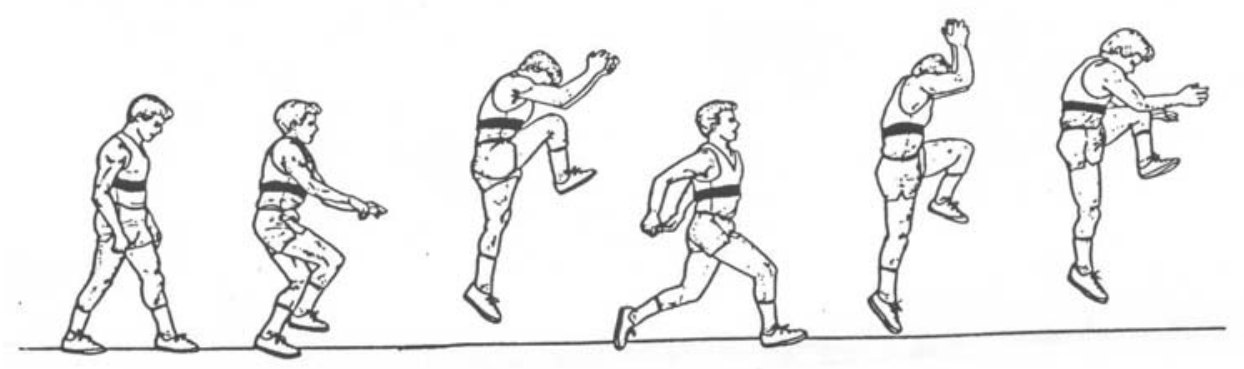

Standing Jump \& Reach - this exercise is especially good for the quadriceps and hip girdle, as well as for the lower back and hamstrings.

Applications: all sports that require leg strength, speed, and quickness (volleyball, diving, swimming, and running)

Start: Stand with feet shoulder width apart.

Action: Squat slightly and explode upward to the highest peak possible. Do not step before jumping.

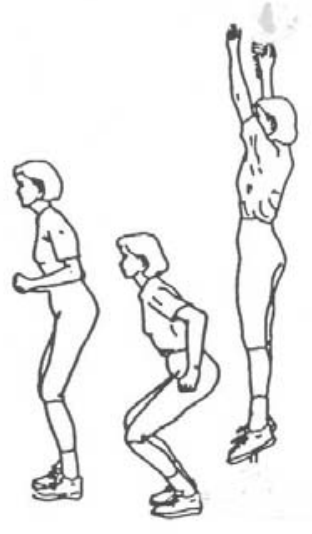


Squat Jumps - the basic drill for developing power (hip flexors, quadriceps, gastrocnemius, hamstring, and gluteus). The primary emphasis in the squat jump is to attain maximum height with every effort. lighting)

Applications: all sports (especially volleyball, jumping events, and weight-

Start: Assume a relaxed upright stance with feet placed shoulder-width apart, placing the palms of the hands against the back of the head. This will minimize the involvement of the arms, thus emphasizing involvement of the legs and hips.

Action: Begin by quickly dropping downward to a half-squat position; immediately check this downward movement and explode upward as high as possible. Upon landing, repeat the sequence; on each repetition, initiate the jumping phase just prior to reaching the half-squat starting position. Work for maximum height with each jump.

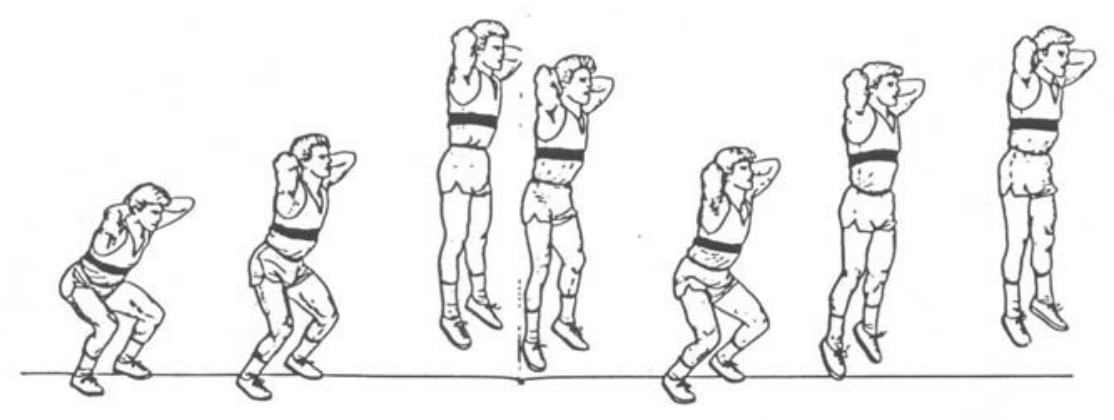

Knee Tuck Jumps - performed as a series of rapid explosive jumps that exercise the hip and leg flexors, gastrocnemius, gluteus, and hamstrings.

Applications: all sports (especially volleyball, diving, weight-lifting, and cycling)

Start: Assume a comfortable upright stance, placing the hands palms down at chest height (or you can use your arms to initiate momentum). Body should be in a straight vertical position and DO NOT BEND AT THE WAIST.

Action: Begin by rapidly dipping down to about the quarter-squat level and immediately explode upward. Drive the knees high toward the chest and attempt to touch them to the palms of the hands or chest. Upon landing, repeat the sequence, each time thinking of driving the knees upward and tucking the feet under the body. Repetitions are performed at a fairly rapid rate with minimum contact on the ground.

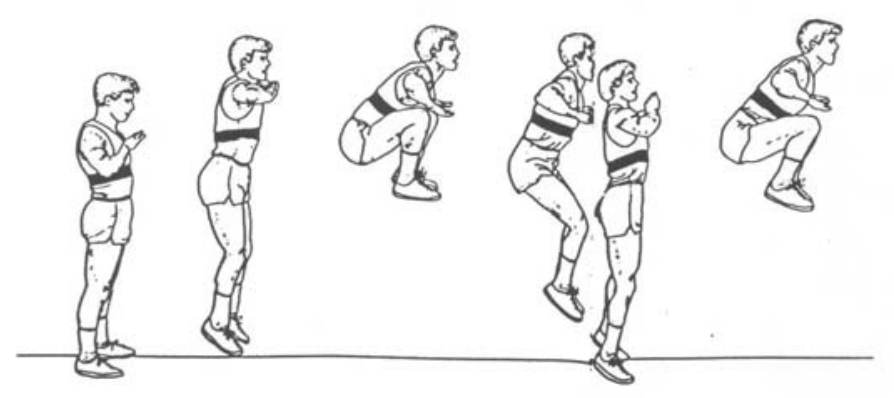


Squat Split Jumps - effects the muscles of the lower back, hamstrings, gluteus, quadriceps, extensors, and flexors. Split jumps are especially for developing stride power. They are also specific to the "split" portion of the clean and jerk.

Applications: running, cross-country skiing, weight lifting, soccer, and volleyball

Start: Assume a stance with one leg extended forward and the other oriented somewhat behind the midline of the body as in executing a long step or stride. The forward leg is flexed with a 90-degree bend at the knee.

Action: Jump as high and straight up as possible. Use the arms in an upward swinging motion to gain additional lift. Upon landing, retain the spread-legged position, bending the knee of the forward leg to absorb the shock. After regaining stability repeat the motion for the required number of time, going as high as possible each time. Upon completing the sequence and rest perform the exercise again with the opposite leg forward.

TIP

- Try for complete extension of the legs and hips when you jump. Remember that the ankle, knee, hip, and trunk play a vital role in achieving maximal height while jumping and in speed when running fast.

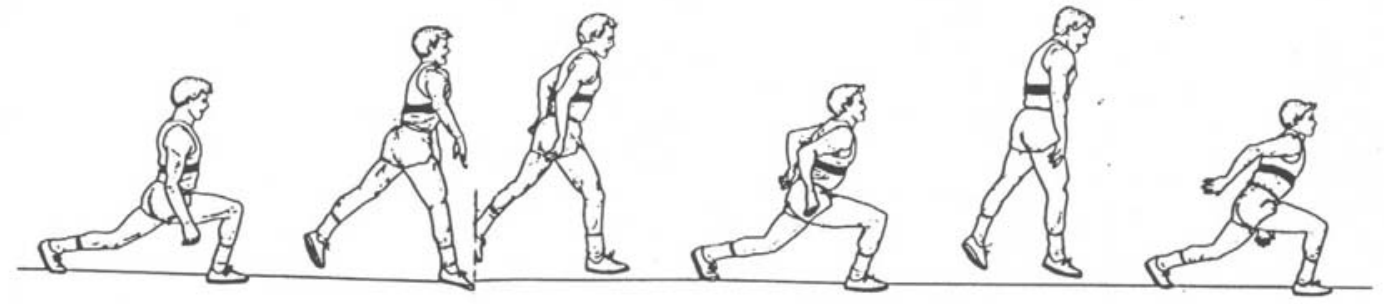

Scissor (Split) Jumps - similar to the split jump, this exercise works the muscles of the lower back, hip extensors, hamstrings, and quadriceps. It is very similar to the split jump except that leg speed is emphasized.

Applications: runners and jumpers

Start: The start position is identical to that of the squat split jump.

Actions: The initial movement of the scissor jump also is identical to that of the squat jump. However, at the apex of the jump the position of the legs is reversed, that is, front to back and back to front. The switching of the legs occurs in midair and must be done very quickly before landing. After landing, the jump is repeated, again reversing the position of the legs. Attainment of maximal vertical height and leg speed are stressed in this exercise.

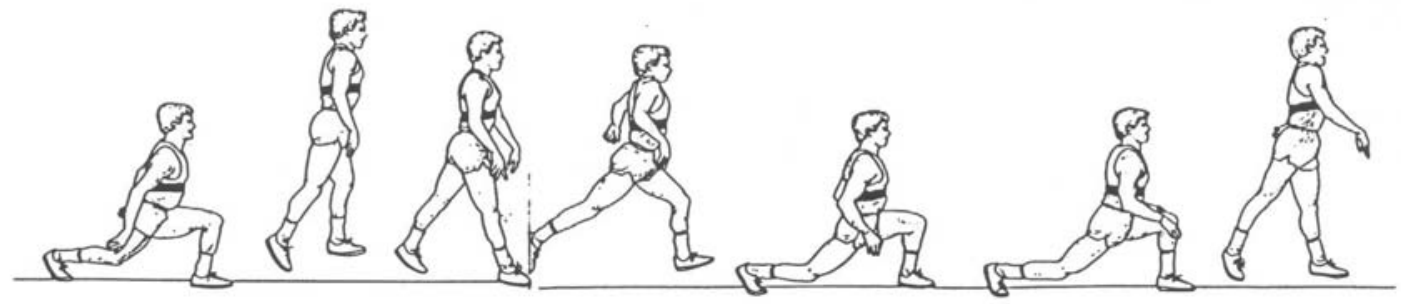


Double Leg Speed Hops (forward) - develops speed and power in the muscles in the leg and hip at a rapid rate (specifically working the gluteus, hamstrings, quadriceps, and gastrocnemius). This is a useful exercise for developing speed and explosiveness.

Applications: running, figure skating, football, swimming, and in-line/speed skating

Start: Assume a relaxed upright stance with back straight, head up, and shoulders slightly forward. Keep arms at sides and bent at 90 degrees with the thumbs up.

Action: Begin by jumping upward as high as possible, flexing the legs completely so as to bring the feet under the buttocks. Emphasize maximum lift by bringing the knees high and forward with each repetition. Upon each landing, jump quickly upward again with the same cycling action of the legs, using the arms to help achieve maximum lift. The action sequence should be executed as rapidly as possible. Work at gaining height and distance.
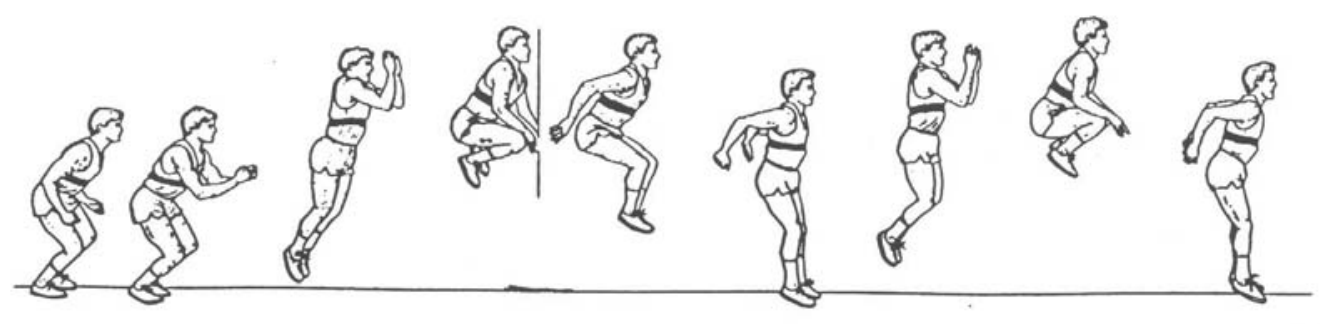

Double Leg Side Hops - specifically develops the thigh abductor muscles, stabilizers of the knees and ankles, and enhances explosive lateral power throughout the legs and hips.

Applications: useful for all activities employing lateral movement (basketball, hockey, tennis, wrestling, and in-line/speed skating

Start: Assume a relaxed upright stance with feet together and pointing straight ahead. Arms should be cocked to enable lift and aid in balance.

Action: From the starting position, jump sideways. Without hesitating change direction by jumping back over in the opposite direction. Continue this back-and-forth sequence. Use the arms in an upward thrusting motion with thumbs up and elbows at 90 degrees.
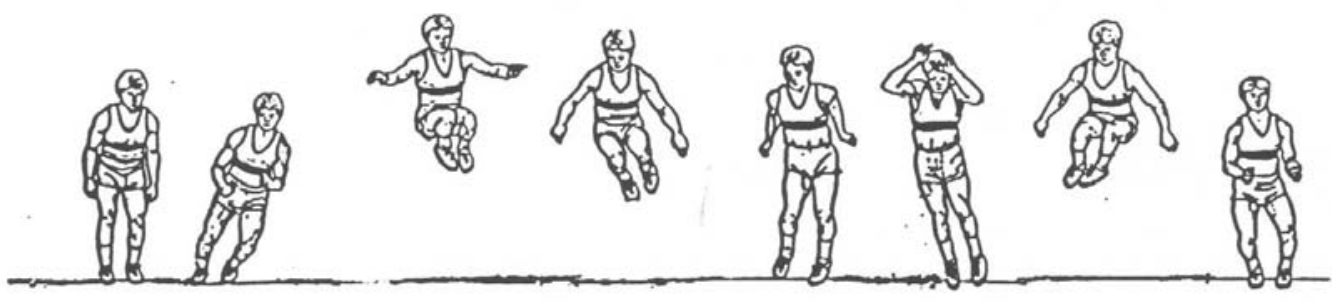
Double Leg Bounding - helps to develop explosive power in leg and hip muscles (gluteus, hamstrings, quadriceps, and gastrocnemius). Arms and shoulder muscles are also directly involved.

Applications: competitive swimming, running, jumping, and weight lifting

Start: Begin with a half-squat. Arms at side with shoulders forward and out over the knees. Keep the back straight and hold the head up.

Action: Jump upward and outward, using extension of the hips and forward thrusting movement of the arms. Try to attain maximum height and distance by fully straightening the body. Upon landing, resume the starting position and initiate the next bound. Emphasize, "reaching for the sky".

TIP

- Remember that with an arm swing, straight arms generate force and short (bent) arms generate speed. Examine your sport or event and see which one is most beneficial for you to use.

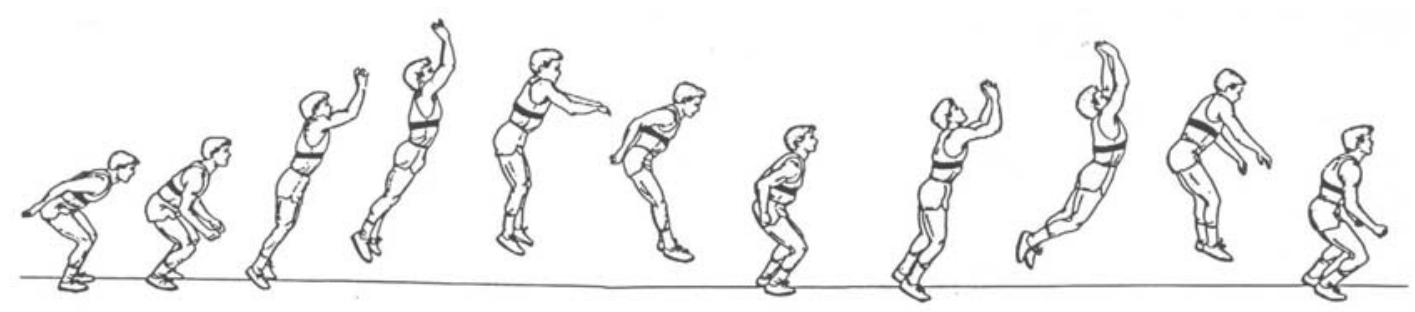

Alternate Leg Bounds - are very similar to the double leg bound in developing explosive leg and hip power. Alternating the legs specifically works the flexors and extensors of the thighs and hips.

Application: enhances running, striding, and sprinting actions (running, jumping, baseball, and cycling)

Start: Assume a comfortable stance with one foot slightly ahead of the other as to initiate a step; arms should be relaxed and at the sides.

Action: Begin by pushing off with the back leg, driving the knee up to the chest and attempting to gain as much height and distance as possible before landing. Quickly extend outward with the driving foot. Either swing the arms in a contra-lateral motion or execute a double arm swing. Repeat the sequence (driving with the other leg) upon landing.

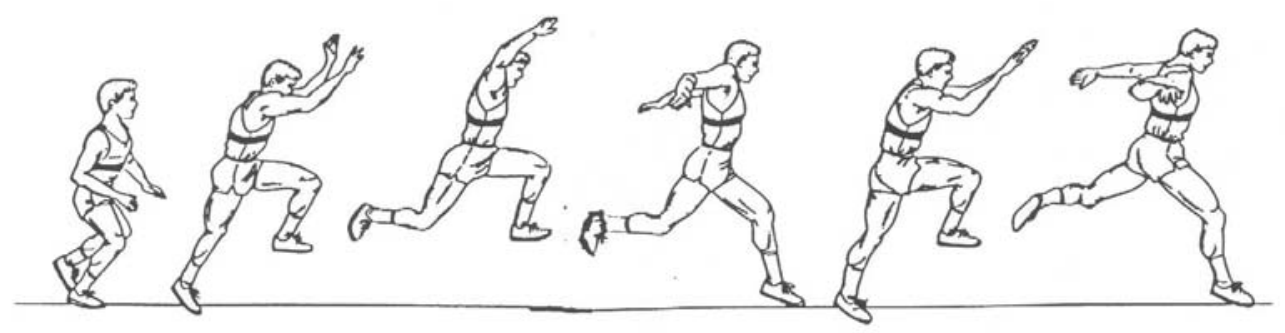


Single Leg Speed Hops (forward) - are similar to the double leg speed hop except that it is done with one leg. This places an overload on the muscle of the hips, legs, and lower back; it incorporates the muscle that stabilizes the knee and ankle.

Applications: running, figure skating, football, swimming, and in-line/speed skating

Start: Assume the same stance as in the double leg speed hop except that one leg should be held in a stationary flexed position throughout the exercise.

Action: Begin the exercise as in the double leg hop but with one (the same) leg at a time.

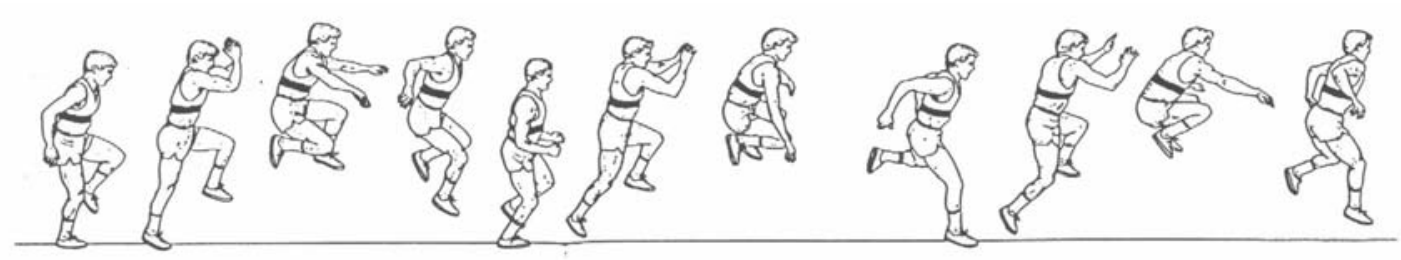

Bounding with Vertical Jump - helps to develop explosive power in the leg and hip muscles, as well as the lower back and hamstrings.

Applications: jumping, running, and volleyball

Start: Stand on one foot.

Action: Do a combination bounding sequence (right-right-left or left-left-right), and then follow immediately with a strong vertical jump. On the third bound, bring the non-bounding foot up to meet the bounding foot so that the jump is off both feet. Use a double arm swing to assist in lifting you vertically. As soon as you land from the vertical jump, complete another bounding sequence following by a vertical jump.

TIP

- Think of your arm action as "punching". This will allow a maximal contribution to overall height of the jump.

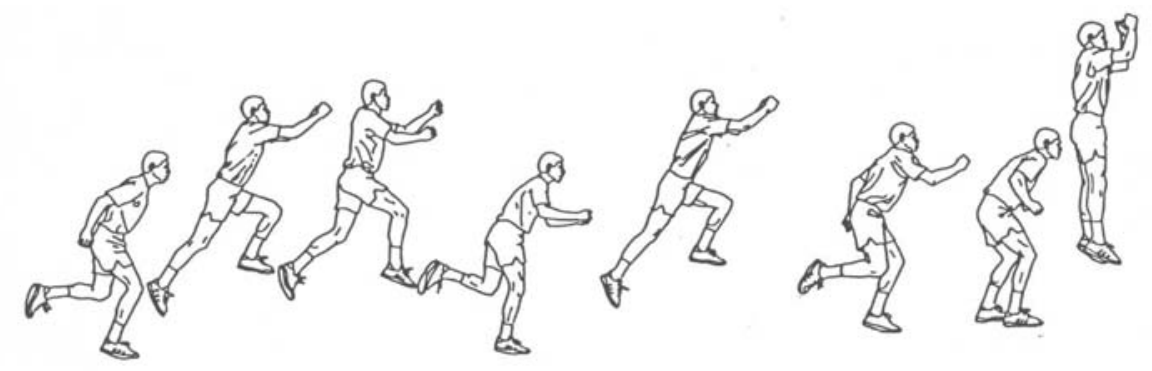


TRAINING VOLUME

Week 1 \& 2

SETS REPS REST CONTACTS

\begin{tabular}{|l|c|c|c|c|}
\hline Two Foot Ankle Hops & 3 & 10 & $0: 30$ & 30 \\
\hline Power Skipping (High Knees) - each leg & 3 & 10 & $0: 45$ & 30 \\
\hline Squat Jumps & 3 & 10 & $0: 45$ & 30 \\
\hline Knee Tuck Jumps & 3 & 10 & $0: 45$ & 30 \\
\hline Heel Tuck Jumps & 3 & 10 & $0: 45$ & 30 \\
\hline THREE MINUTE BREAK & & & & \\
\hline Squat Split Jumps (each leg) & 3 & 10 & $0: 45$ & 60 \\
\hline Double Leg Speed Hops - forward & 3 & 10 & $0: 45$ & 30 \\
\hline Double Leg Bounds & 3 & 10 & $0: 45$ & 30 \\
\hline Double Leg Side Hops & 3 & 10 & $0: 45$ & 30 \\
\hline Standing Jump \& Reach & 3 & 10 & $0: 45$ & 30 \\
\hline TOTAL CONTACTS & & & & 360 \\
\hline
\end{tabular}

Week $3,4, \& 5$

SETS REPS REST CONTACTS

\begin{tabular}{|l|c|c|c|c|}
\hline Two Foot Ankle Hops & 3 & 15 & $0: 45$ & 45 \\
\hline Power Skipping (High Knees) & 3 & 20 & $0: 45$ & 120 \\
\hline Squat Jumps & 3 & 15 & $0: 45$ & 45 \\
\hline Knee Tuck Jumps & 3 & 15 & $0: 45$ & 45 \\
\hline Heel Kick Jumps & 3 & 15 & $0: 45$ & 45 \\
\hline THREE MINUTE BREAK & & & & \\
\hline Scissors Split Jumps & 3 & 15 & $0: 45$ & 60 \\
\hline Alternate Leg Bounds & 3 & 20 & $0: 45$ & 30 \\
\hline Double Leg Side Hops & 4 & 10 & $0: 45$ & 40 \\
\hline Single Leg Speed Hops - forward (each leg) & 4 & 10 & $0: 45$ & 80 \\
\hline Double Leg Bounds with Vertical Jump & 4 & 10 & $0: 45$ & 80 \\
\hline TOTAL CONTACTS & & & & 590 \\
\hline
\end{tabular}

Week $6,7, \& 8$

\begin{tabular}{|l|c|c|c|c|}
\hline $\begin{array}{l}\text { Two Foot Ankle Hops (feet shoulder width } \\
\text { apart) }\end{array}$ & 4 & 15 & $0: 30$ & 60 \\
\hline Squat Jumps & 4 & 15 & $0: 45$ & 60 \\
\hline Knee Tuck Jumps & 3 & 15 & $0: 45$ & 45 \\
\hline Heel Kick Jumps & 3 & 15 & $0: 45$ & 45 \\
\hline Double Leg Side Hops & 4 & 10 & $0: 30$ & 40 \\
\hline THREE MINUTE BREAK & & & & \\
\hline Split Jumps (each leg) & 4 & 10 & $0: 45$ & 80 \\
\hline Alternate Leg Bounds & 4 & 20 & $0: 45$ & 80 \\
\hline Single Leg Speed Hops - forward (each leg) & 4 & 15 & $0: 45$ & 120 \\
\hline Double Leg Bounds with Vertical Jump & 5 & 10 & $0: 45$ & 100 \\
\hline CONTACT & & & & 630 \\
\hline
\end{tabular}


APPENDIX J

PEAK POWER OUTPUT - SARGENT VERTICAL JUMP TEST PROTOCOL 


\section{PEAK POWER OUTPUT - SARGENT VERTICAL JUMP TEST}

Description: measures the difference between a person's standing reach and maximum jump height is recorded in centimeters.

1. Explain to the subject that we are going to assess her explosive power.

2. Subject stands facing the vertical jump board with both feet flat on the floor and toes touching the base of the wall. (State to the subject that the hand you mark with must be the hand you reach with when testing)

3. Subject dips her fingers in chalk and then extend her arm up as high as possible (with feet flat). This equals her standing height.

4. Subject then turns perpendicular to the vertical jump board.

5. Instruct the subject to squat and jump as high as possible and make a mark on the vertical jump board at the peak of the jump.

6. As the subject takes off to jump, say, "EXPLODE” out loud.

7. The subject will rest for 30 seconds and perform the jump again (steps 1-5).

8. The vertical jump score is the greatest difference between the two marks; standing height and jump height (record in centimeters).

10. A total of three (3) vertical jumps are obtained. Record all three jumps on the data sheet. 
APPENDIX K

PEAK TORQUE PRODUCTION - ISOKINETIC STRENGTH TESTING PROTOCOL 


\section{PEAK TORQUE PRODUCTION - ISOKINETIC STRENGTH TESTING}

Description: assessed peak torque production miometrically and pliometrically.

1. Turn on both power switches (power strip and switch on right side)

2. Seat the subject in the Biodex chair (test dominant leg only)

- Ask her if she knows her dominant leg, if not use the side of the writing hand

- Place a note on the data sheet for which leg is used - use the same leg every time

3. Make sure Database Management is set on (under Utilities)

4. $\quad$ Enter New Patient information and Save/Select patient (F1)

- Include my initials after Subject's last name (i.e., Williams-LR)

- When you mid-/posttest go to database management and click on get patient. Type in the last name followed by LR (the computer will bring them up)

5. Set up the subject as follows:

- Align axis of power head and lever arm with the anatomical axis of knee by moving power head machine up and down. Will have to adjust: the seat forward and backward and the ankle pad. The ankle make should contact the tibia just above the malleous (ankle)

i. Record the machine height

ii. Record the ankle height

iii. Record the seat angle

iv. Record the seat distance (under the chair)

** You will record the measurements once and input it on the subject's data sheet** We will refer back to the measurements for each testing to keep the measurements, angles, and heights constant**

- Tighten Velcro straps on the lower leg, thigh, torso, and shoulder

6. $\quad$ Select Isokinetic, Test, New Test, 3 Speed Bilateral, Extension/Flexion and Concentric/Concentric

7. Make sure the machine is in the Setup mode and sensitivity is set at $\mathrm{C}$ and that the ROM for $1 \& 2$ is set at 90 degree range

8. $\quad$ Scroll up and select Formats and press return to enter (hit F6 and then F1)

$\begin{array}{lccl}\text { Uninvolved SLOW } 60 \mathrm{deg} / \mathrm{sec} & 8 & 60 & 60 \text { (Warm-up) } \\ \text { Uninvolved MEDIUM 60 deg/sec } & 6 & 60 & 60 \\ \text { Uninvolved FAST } 60 \mathrm{deg} / \mathrm{sec} & 12 & 60 & 60\end{array}$

9. Press F1 with the top line of the protocol highlighted to select the protocol

10. Set the Anatomical Reference to 90.0 degrees if not set

11. Select Verify Range, have subject move to maximal extension and press Limit Set 1, have subject move to maximal flexion and press Limit Set 2 
12. Select Limb Weight, have subject move to 30 degrees minus maximal extension press the red plunger button (stop button), allow limb weight to settle (tell subjects to relax and press Return to accept limb weight

- Record the Limb weight on the data sheet

13. Press the stop button that is on the control panel

14. Accept the values for Scaling Value: Autoscale

15. Change the Scoring Window to $85 \%$

16. Accept the value for Number of Reps

17. Once Run Test is highlighted, Switch to Isokinetic Test Mode, turn speed dials to the appropriate setting (under Isokinetic Setting), and press Start

18. Press any key to begin test

19. During warm-up instruct to do a sub maximal performance

- Remind the subjects that they want to kick with the most force and power (KICK FAST \& HARD)

- Give the subject 2:00 minute to rest between warm-up and sets

- Have the subjects hold onto the chair seat

20. Save test - press F1

21. Get results - go to Reports, Evaluation, General Reports, and then press F4

22. To print - press F1

23. To measure Eccentric/Eccentric

23. Return to Setup mode

25. Select Isokinetic, Test, New Test, 3 Speed bilateral, Extension/Flexion and Eccentric/Eccentric and proceed with the same procedures for measuring concentric/concentric; steps 7-16.

26. Once Run Test is highlighted, Switch to Eccentric Test Mode, turn speed dials to the appropriate setting (under Eccentric Setting), make sure Torque Limits are set at there maximum and then press Start and High-Speed Enabler at the same time.

27. Press any key to begin test

28. During warm-up instruct to do a sub maximal performance

- Remind the subjects that they want to hyper-flex and extend to start the lever arm and then resist the movement of the arm

- Give the subject 22:00 minute to rest between warm-up and sets

- Have the subjects hold onto the chair seat

29. Print Save test - press F1

30. Get results - go to Reports, Evaluation, General Reports, and then press F4

31. To print - press F1 


\section{APPENDIX L}

VELOCITY - 40 METER SPRINT PROTOCOL 


\section{VELOCITY - 40 METER SPRINT}

Description: time to run a set distance (40-m) is recorded in seconds.

1. Explain to the subject that we are going to measure the time it takes to complete a 40-meter sprint.

2. Each sprint will begin with a 3-point stance position

- the subjects position the front of either the right or left foot on a line 30 $\mathrm{cm}$ behind the start line and touches the same line with either the left hand, if the right foot was the lead foot or the right hand, if the left foot was the lead foot

3. The opposite arm will be held off the floor and behind the body. This arm will serve as the swing arm to help gain momentum.

4. Subjects are instructed to "SPRINT AS FAST AS POSSIBLE" over the distance and started when they were given the cue or command.

6. Two timers recorded each sprint

7. The subjects performed three sprints (with a 3-minute rest) between each sprint. Recorded all three sprint times on the data sheet. 


\section{APPENDIX M}

MUSCLE SORENESS SCALE / PROTOCOL 


\section{MUSCLE SORENESS SCALE}

Description: measures the perception of muscle soreness of the rectus femoris (quadriceps), biceps femoris (hamstrings), and gastrocnemius (calf) muscles.

1. Explain to the subject that we are going to assess her perception of muscle soreness of the specific muscles.

2. Show the subject the muscle soreness scale.

3. Ask the subject to rate her perception of muscle soreness for her quadriceps, hamstrings, and calf muscles.

4. Please state that:

- this is pain, tenderness, and soreness specifically to the specific muscle

- a $1=$ no pain, tenderness, and soreness at all, 5 = some pain, tenderness, and soreness but mobile, $10=$ extreme pain, tenderness, and soreness unable to be mobile

- $\quad$ point to the specific muscle on the scale and on their body 
The muscle soreness scales were adapted and revised from Clarkson \& Tremblay (19).

\section{MUSCLE SORENESS}

\section{Rectus Femoris}
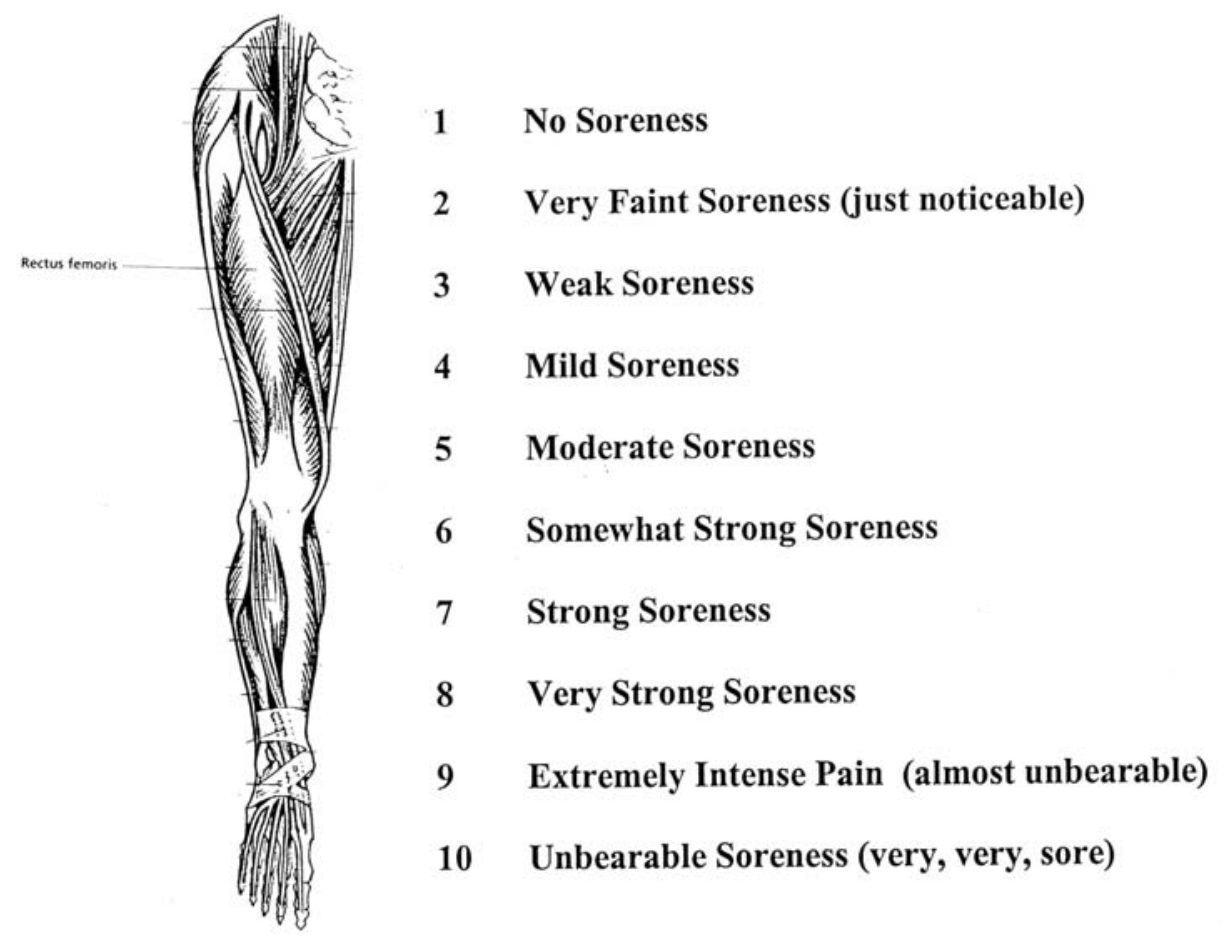


\section{MUSCLE SORENESS}

\section{Biceps Femoris \& Gastrocnemius}

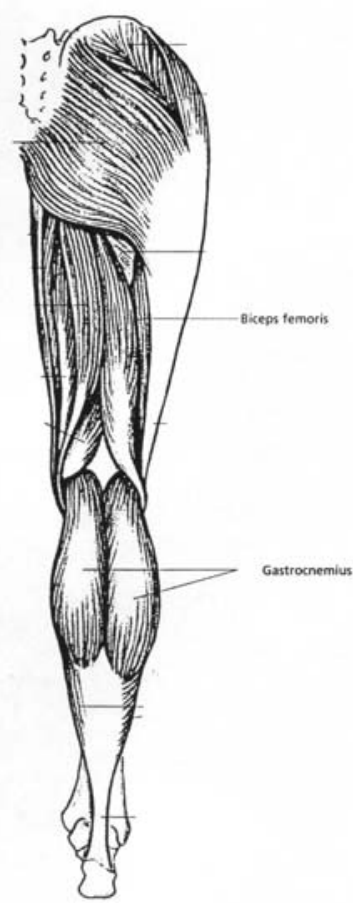

$1 \quad$ No Soreness

2 Very Faint Soreness (just noticeable)

3 Weak Soreness

$4 \quad$ Mild Soreness

$5 \quad$ Moderate Soreness

6 Somewhat Strong Soreness

$7 \quad$ Strong Soreness

$8 \quad$ Very Strong Soreness

9 Extremely Intense Pain (almost unbearable)

10 Unbearable Soreness (very, very, sore) 


\section{APPENDIX N}

PAIN SENSITIVITY - ALGOMETER PROTOCOL 


\section{PAIN SENSITIVITY - ALGOMETER}

Description: assessment of pain sensitivity to of the rectus femoris (quadriceps), biceps femoris (hamstrings), and gastrocnemius (calf) muscles

1. Explain to the subject that you are going to assess the pain sensitivity of each specific muscle of her dominant leg.

2. State the following:

- "I'm going to measure your pain sensitivity - that is how much pressure will induce discomfort."

- Show them algometer and explain, "I am going to increase pressure slowly with this device. Please say "YES" when you feel any discomfort and pain. I will stop the pressure as soon as you speak so that it will not hurt you.

3. Place the subject in a prone position.

- Relaxation of the muscles is essential for accurate measurement

- Ask the subject to relax and reassure her that the procedure will not hurt.

4. Record the mark for each muscle on the datasheet and apply the pressure gauge to the skin:

- Belly of the rectus femoris - midpoint between the inguinal crease and the proximal border of the patella

- Lateral aspect of the biceps femoris muscle belly - midpoint between the gluteus furrow to the posture side of the patella

- Medical aspect of gastrocnemius muscle belly

5. Pressure must be applied at a constant rate of $1 \mathrm{~kg} / \mathrm{sec}$.

- The research technicians achieved this by counting one and thousand, two and thousand three and thousand and so on while increasing the pressure evenly and visually watching the pressure gauge increase at $1 \mathrm{~kg} / \mathrm{sec}$.

* All of the research technicians were thoroughly trained with eh algometer prior to the study.

6. Once you record the pressure on the data sheet, press the release button.

7. Perform this task at each specific muscle site. 
Algometer (Algometer FDK40, Scrip Inc.; Peoria, IL)

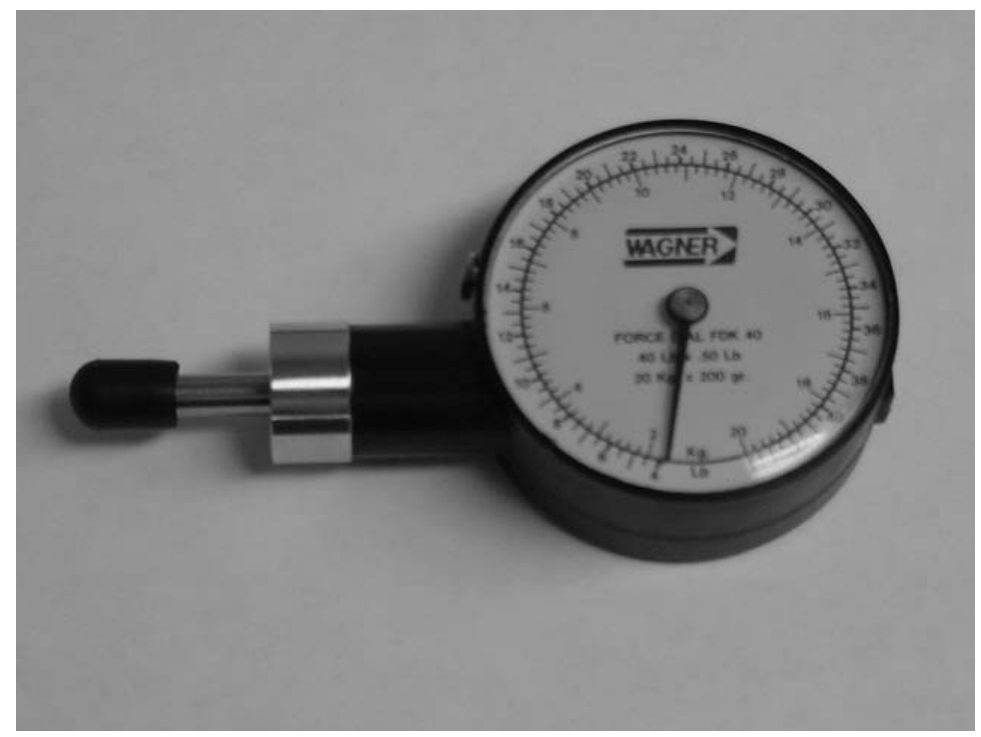

Pain sensitivity sites (rectus femoris, biceps femoris, and gastrocnemius)
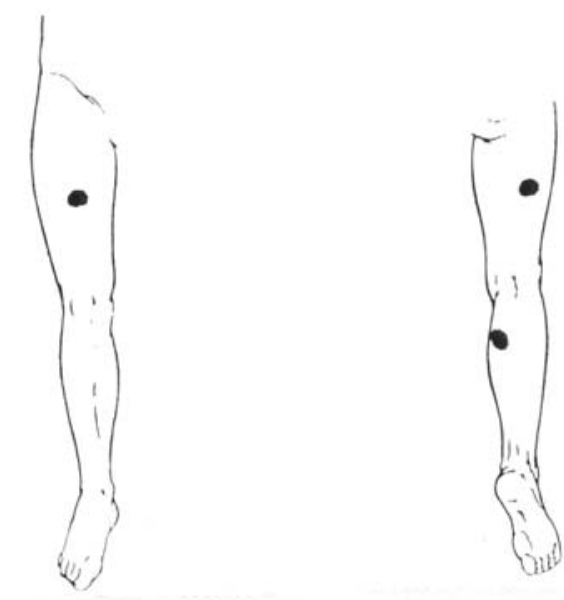

Anterior

\section{Posterior}


APPENDIX O

BODY COMPOSITION ASSESSMENT PROTOCOL 


\section{BODY COMPOSITION - BOD POD}

Description: measurement of body density; percent body fat and percent lean mass.

1. Insure the subject is appropriately dressed and ready for testing (bathing suit or body-conforming clothing). The subject should be wearing minimal clothing that fits as snugly as possible. The subjects should wear a swim cap to compress their hair.

2. Calibrating the Bod Pod (empty). The first calibration establishes a "zero" baseline. Simply following instruction as they appear on the computer screen. Do not touch or lean against the Bod Pod during any part of the testing process. Ignore the prompt that asks you to install a new filter and breathing tube, as this is down when measuring lung volume and we are using predicted volumes.

3. Entering subject data. Enter information requested using the $\langle$ ENTER $\rangle$ key to move subject on the Stadiometer to determine this value. Once all the information is entered, press $\langle$ ALT $\rangle+\langle$ ENTER $>$ to submit the screen.

4. Calibrating the Bod Pod (with white cylinder). Again, follow the instructions as they appear on the computer screen. As the cylinder is of a known volume, any dents will alter this volume. USE EXTREME CARE WITH THE CYLINDER DO NOT DROP IT!

5. Weighing the subject. Weigh subjects on the scale attached to the Bod Pod. Have the subject removed all extra clothing and shoes. Follow the prompt as they appear on the computer screen.

6. Measure the body volume of the subject. Have the subject sit in the Bod Pod with their hands in their lap, in a relaxed and comfortable position. Make sure the swim cap is on. Instruct the subject to relax, breathe normally and sit as still as possible. Identify the blue "PANIC BUTTON" on the lower left side of the seat, and explain that it will enable the subject to terminate the test any time in case of an emergency.

7. Conducting a second test. Instruct the subjects to keep still between tests. It is necessary that they are in the same position for both tests! If the data from the second test in inconsistent with the first test, you are asked to conduct a third test. Follow the instructions as they appear on the computer screen.

8. Print out the final report/results. 


\section{APPENDIX P}

ANTHROPOMETRICAL MEASUREMENT PROTOCOL 


\section{ANTHROPOMETRICAL MEASUREMENTS}

Description: measurement of height and leg length to rule out subjects with a leg length discrepancy $\geq 3 \mathrm{~cm}$.

1. Height is the linear measurement of the distance from the standing surface to the top (vertex) of the skull. It is measured with the subject in the standard erect posture, without shoes. Weight is evenly distributed between feet, heels together, arms hanging relaxed at the side, and the head position straight ahead in the horizontal plane.

2. Leg length is a linear measurement of the distance from the great trochanter of the femur to the lateral malleolus of the fibula. It is measured with the subject in the standard erect posture, without shoes, while heels are together.

3. Leg length is the linear measurement of the distance from the lateral condoyle of the tibia to the lateral malleolus of the fibula. It is measured with the subject in the standard erect posture, without shoes, and heels are together.

4. Thigh length is the linear measurement of the distance from the greater trochanter of the femur to the lateral epicondyle of the femur. It is measured with the subject in the standard erect posture, without shoes, and heels are together. 


\section{APPENDIX Q}

RECTUS FEMORIS MUSCLE SORENESS 


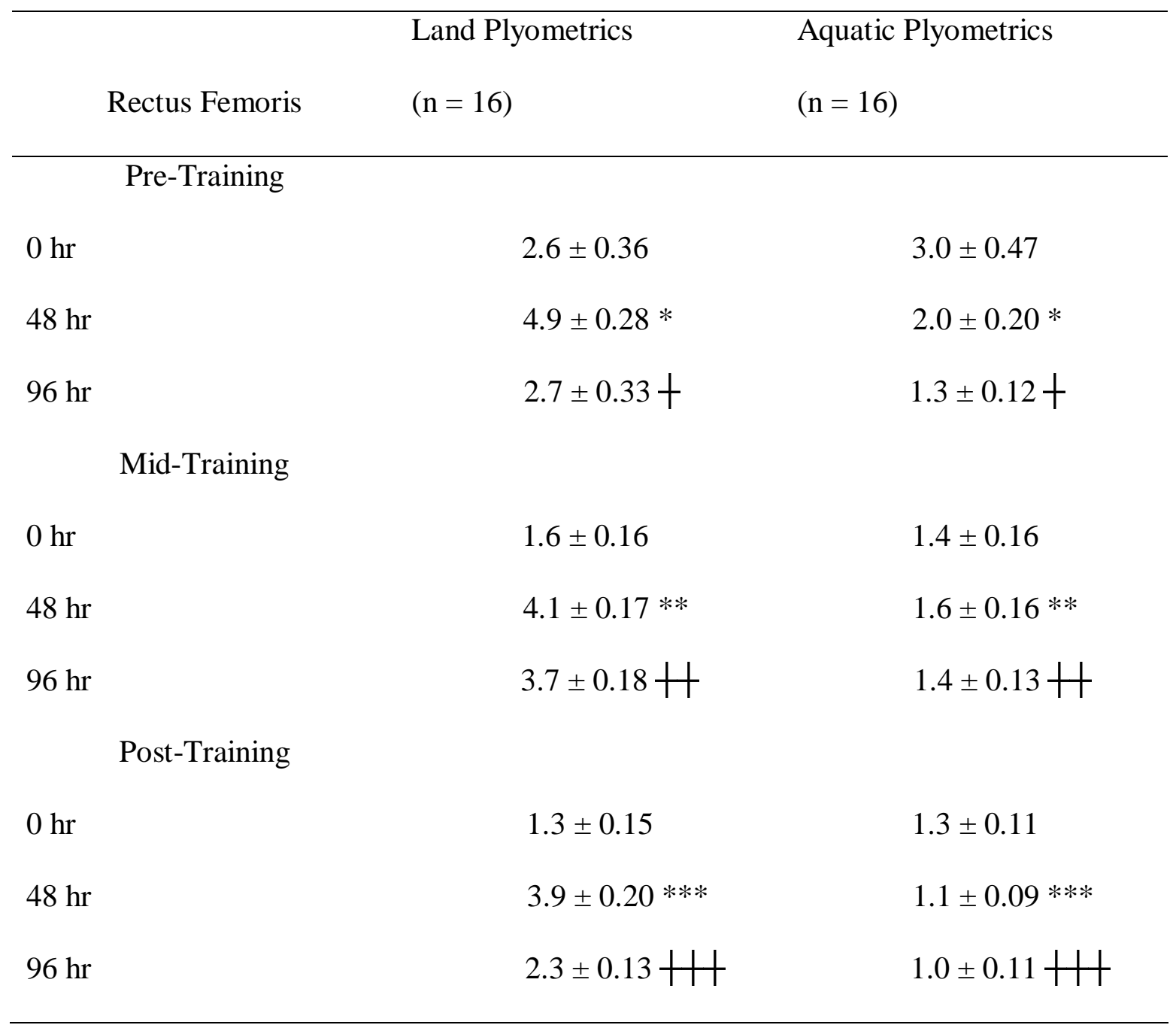

* Baseline: $48 \mathrm{hr}$ land plyometrics $>$ aquatic plyometrics, $p \leq 0.001$.

+ Baseline: $96 \mathrm{hr}$ land plyometrics $>$ aquatic plyometrics, $p \leq 0.001$.

$* *$ Increase in training intensity 1: $48 \mathrm{hr}$ land plyometrics > aquatic plyometrics, $p \leq$ 0.001 .

† Increase in training intensity 1: $96 \mathrm{hr}$ land plyometrics $>$ aquatic plyometrics, $p \leq$ 0.001 .

*** Increase in training intensity $2: 48 \mathrm{hr}$ land plyometrics $>$ aquatic plyometrics, $p \leq$ 0.001 .

十 Increase in training intensity 2: $96 \mathrm{hr}$ land plyometrics $>$ aquatic plyometrics, $p \leq$ 0.001 .

Table A.1 Muscle soreness perception of the rectus femoris muscle. 


\section{APPENDIX R}

BICEPS FEMORIS MUSCLE SORENESS 


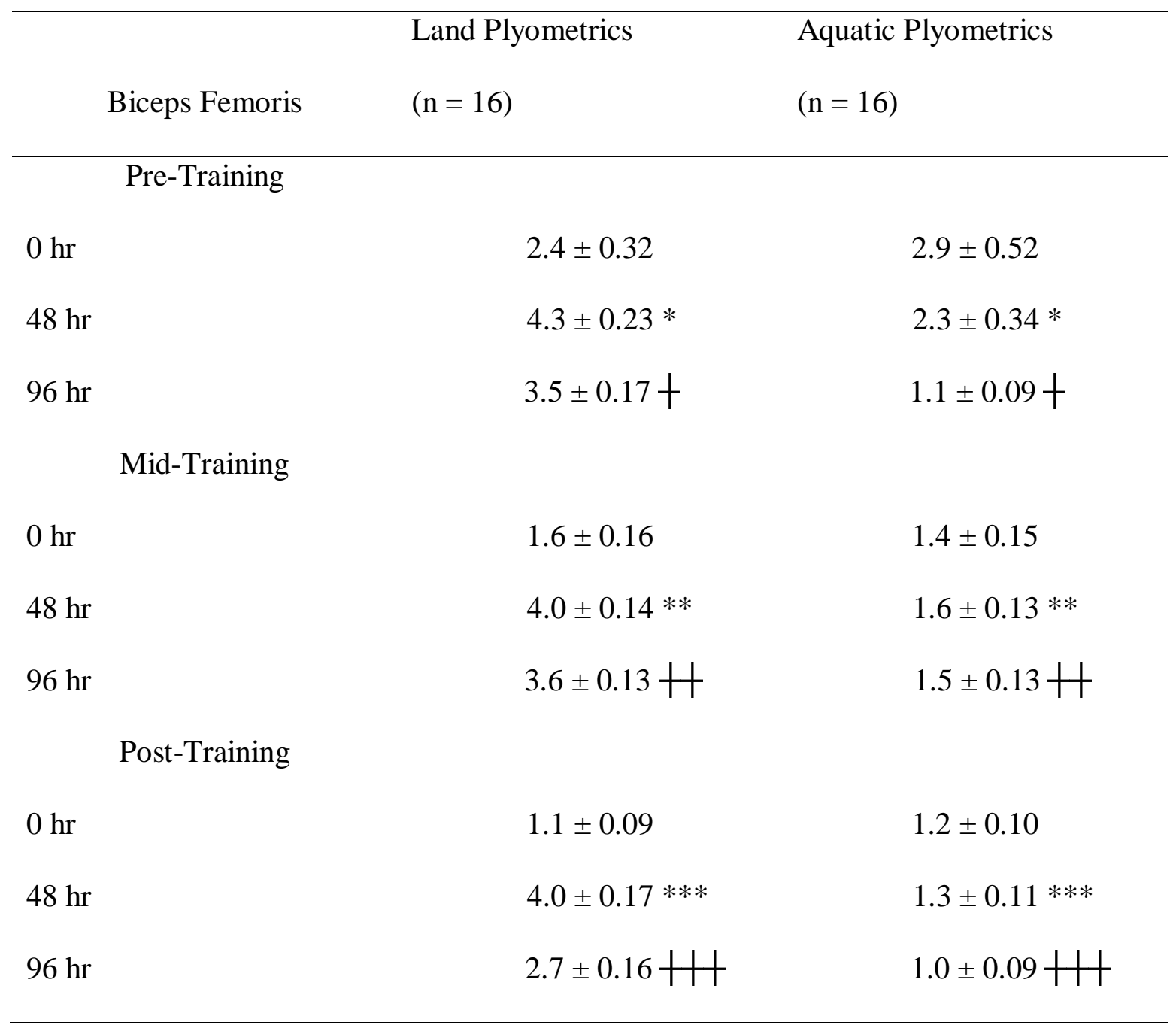

* Baseline: $48 \mathrm{hr}$ land plyometrics $>$ aquatic plyometrics, $p \leq 0.001$.

+ Baseline: $96 \mathrm{hr}$ land plyometrics $>$ aquatic plyometrics, $p \leq 0.001$.

$* *$ Increase in training intensity 1: $48 \mathrm{hr}$ land plyometrics > aquatic plyometrics, $p \leq$ 0.001 .

† Increase in training intensity 1: $96 \mathrm{hr}$ land plyometrics $>$ aquatic plyometrics, $p \leq$ 0.001 .

*** Increase in training intensity $2: 48 \mathrm{hr}$ land plyometrics $>$ aquatic plyometrics, $p \leq$ 0.001 .

十 Increase in training intensity 2: $96 \mathrm{hr}$ land plyometrics $>$ aquatic plyometrics, $p \leq$ 0.001 .

Table A.2 Muscle soreness perception of the biceps femoris muscle. 


\section{APPENDIX S}

GASTROCNEMIUS MUSCLE SORENESS 


\section{Land Plyometrics}

Gastrocnemius $(\mathrm{n}=16)$

\section{Pre-Training}

$0 \mathrm{hr}$

$48 \mathrm{hr}$

$96 \mathrm{hr}$

Mid-Training

$$
2.3 \pm 0.30
$$

$5.3 \pm 0.27 *$

$4.7 \pm 0.23+$
$2.2 \pm 0.31$

$2.4 \pm 0.30 *$

$1.4 \pm 0.16+$
$0 \mathrm{hr}$

$48 \mathrm{hr}$

$96 \mathrm{hr}$
$1.7 \pm 0.18$

$4.5 \pm 0.19 * *$

$4.8 \pm 0.17$ 十
$1.7 \pm 0.24$

$1.8 \pm 0.17 * *$

$1.4 \pm 0.18+$

Post-Training

$0 \mathrm{hr}$

$48 \mathrm{hr}$

$96 \mathrm{hr}$
$1.1 \pm 0.09$

$4.7 \pm 0.12 * * *$

$3.8 \pm 0.17$ 十十
$1.4 \pm 0.18$

$1.4 \pm 0.15 * * *$

* Baseline: $48 \mathrm{hr}$ land plyometrics $>$ aquatic plyometrics, $p \leq 0.001$.

+ Baseline: $96 \mathrm{hr}$ land plyometrics $>$ aquatic plyometrics, $p \leq 0.001$.

** Increase in training intensity 1: $48 \mathrm{hr}$ land plyometrics > aquatic plyometrics, $p \leq$ 0.001 .

H Increase in training intensity 1: $96 \mathrm{hr}$ land plyometrics $>$ aquatic plyometrics, $p \leq$ 0.001 .

*** Increase in training intensity $2: 48 \mathrm{hr}$ land plyometrics $>$ aquatic plyometrics, $p \leq$ 0.001 .

+ Increase in training intensity 2: $96 \mathrm{hr}$ land plyometrics $>$ aquatic plyometrics, $p \leq$ 0.001 .

Table A.3 Muscle soreness perception of the gastrocnemius muscle. 


\section{APPENDIX T}

RECTUS FEMORIS PAIN SENSITIVITY 


\begin{tabular}{|c|c|c|c|}
\hline \multirow{2}{*}{\multicolumn{2}{|c|}{ Rectus Femoris }} & \multirow{2}{*}{$\begin{array}{l}\text { Land Plyometrics } \\
(\mathrm{n}=16)\end{array}$} & \multirow{2}{*}{$\begin{array}{l}\text { Aquatic Plyometrics } \\
(\mathrm{n}=16)\end{array}$} \\
\hline & & & \\
\hline & Pre-Training & & \\
\hline $0 \mathrm{hr}$ & & $5.1 \pm 0.27$ & $5.1 \pm 0.23$ \\
\hline $48 \mathrm{hr}$ & & $2.6 \pm 0.17$ & $4.1 \pm 0.21$ \\
\hline \multirow[t]{2}{*}{$96 \mathrm{hr}$} & & $3.1 \pm 0.09$ & $4.6 \pm 0.23$ \\
\hline & Mid-Training & & \\
\hline $0 \mathrm{hr}$ & & $5.3 \pm 0.22$ & $5.3 \pm 0.27$ \\
\hline $48 \mathrm{hr}$ & & $2.7 \pm 0.14$ & $4.4 \pm 0.23$ \\
\hline \multirow[t]{2}{*}{$96 \mathrm{hr}$} & & $3.4 \pm 0.14$ & $4.8 \pm 0.22$ \\
\hline & Post-Training & & \\
\hline $0 \mathrm{hr}$ & & $5.3 \pm 0.23$ & $5.2 \pm 0.23$ \\
\hline $48 \mathrm{hr}$ & & $3.3 \pm 0.10$ & $4.5 \pm 0.22$ \\
\hline $96 \mathrm{hr}$ & & $3.7 \pm 0.10$ & $4.8 \pm 0.22$ \\
\hline
\end{tabular}

Table A.4 Pain sensitivity of the rectus femoris muscle. 
APPENDIX U

BICEPS FEMORIS PAIN SENSITIVITY 


\begin{tabular}{|c|c|c|c|}
\hline \multirow{2}{*}{\multicolumn{2}{|c|}{ Biceps Femoris }} & \multirow{2}{*}{$\begin{array}{l}\text { Land Plyometrics } \\
(\mathrm{n}=16)\end{array}$} & \multirow{2}{*}{$\begin{array}{l}\text { Aquatic Plyometrics } \\
(\mathrm{n}=16)\end{array}$} \\
\hline & & & \\
\hline & Pre-Training & & \\
\hline $0 \mathrm{hr}$ & & $5.0 \pm 0.30$ & $5.1 \pm 0.29$ \\
\hline $48 \mathrm{hr}$ & & $2.7 \pm 0.12$ & $4.1 \pm 0.29$ \\
\hline \multirow[t]{2}{*}{$96 \mathrm{hr}$} & & $3.2 \pm 0.11$ & $4.6 \pm 0.29$ \\
\hline & Mid-Training & & \\
\hline $0 \mathrm{hr}$ & & $5.2 \pm 0.26$ & $5.0 \pm 0.27$ \\
\hline $48 \mathrm{hr}$ & & $2.9 \pm 0.12$ & $4.1 \pm 0.26$ \\
\hline \multirow[t]{2}{*}{$96 \mathrm{hr}$} & & $3.7 \pm 0.18$ & $4.6 \pm 0.27$ \\
\hline & Post-Training & & \\
\hline $0 \mathrm{hr}$ & & $5.1 \pm 0.27$ & $5.1 \pm 0.28$ \\
\hline $48 \mathrm{hr}$ & & $2.9 \pm 0.19$ & $4.3 \pm 0.29$ \\
\hline $96 \mathrm{hr}$ & & $3.6 \pm 0.19$ & $4.8 \pm 0.28$ \\
\hline
\end{tabular}

Table A.5 Pain sensitivity of the biceps femoris muscle. 


\section{APPENDIX V}

GASTROCNEMIUS PAIN SENSITIVITY 


\begin{tabular}{|c|c|c|c|}
\hline \multirow{2}{*}{\multicolumn{2}{|c|}{ Gastrocnemius }} & \multirow{2}{*}{$\begin{array}{l}\text { Land Plyometrics } \\
(\mathrm{n}=16)\end{array}$} & \multirow{2}{*}{$\begin{array}{l}\text { Aquatic Plyometrics } \\
(\mathrm{n}=16)\end{array}$} \\
\hline & & & \\
\hline & Pre-Training & & \\
\hline $0 \mathrm{hr}$ & & $4.0 \pm 0.21$ & $3.9 \pm 0.19$ \\
\hline $48 \mathrm{hr}$ & & $1.8 \pm 0.19$ & $3.3 \pm 0.18$ \\
\hline \multirow[t]{2}{*}{$96 \mathrm{hr}$} & & $2.3 \pm 0.06$ & $3.6 \pm 0.18$ \\
\hline & Mid-Training & & \\
\hline $0 \mathrm{hr}$ & & $4.2 \pm 0.31$ & $4.0 \pm 0.18$ \\
\hline $48 \mathrm{hr}$ & & $2.1 \pm 0.17$ & $3.2 \pm 0.14$ \\
\hline \multirow[t]{2}{*}{$96 \mathrm{hr}$} & & $2.7 \pm 0.14$ & $3.6 \pm 0.17$ \\
\hline & Post-Training & & \\
\hline $0 \mathrm{hr}$ & & $4.2 \pm 0.31$ & $4.0 \pm 0.17$ \\
\hline $48 \mathrm{hr}$ & & $2.3 \pm 0.16$ & $3.4 \pm 0.15$ \\
\hline $96 \mathrm{hr}$ & & $2.6 \pm 0.08$ & $3.7 \pm 0.15$ \\
\hline
\end{tabular}

Table A.6 Pain sensitivity of the gastrocnemius muscle. 


\section{APPENDIX W}

DATA COLLECTION SHEET 
ID \#

Dominant Leg

DATA SHEET

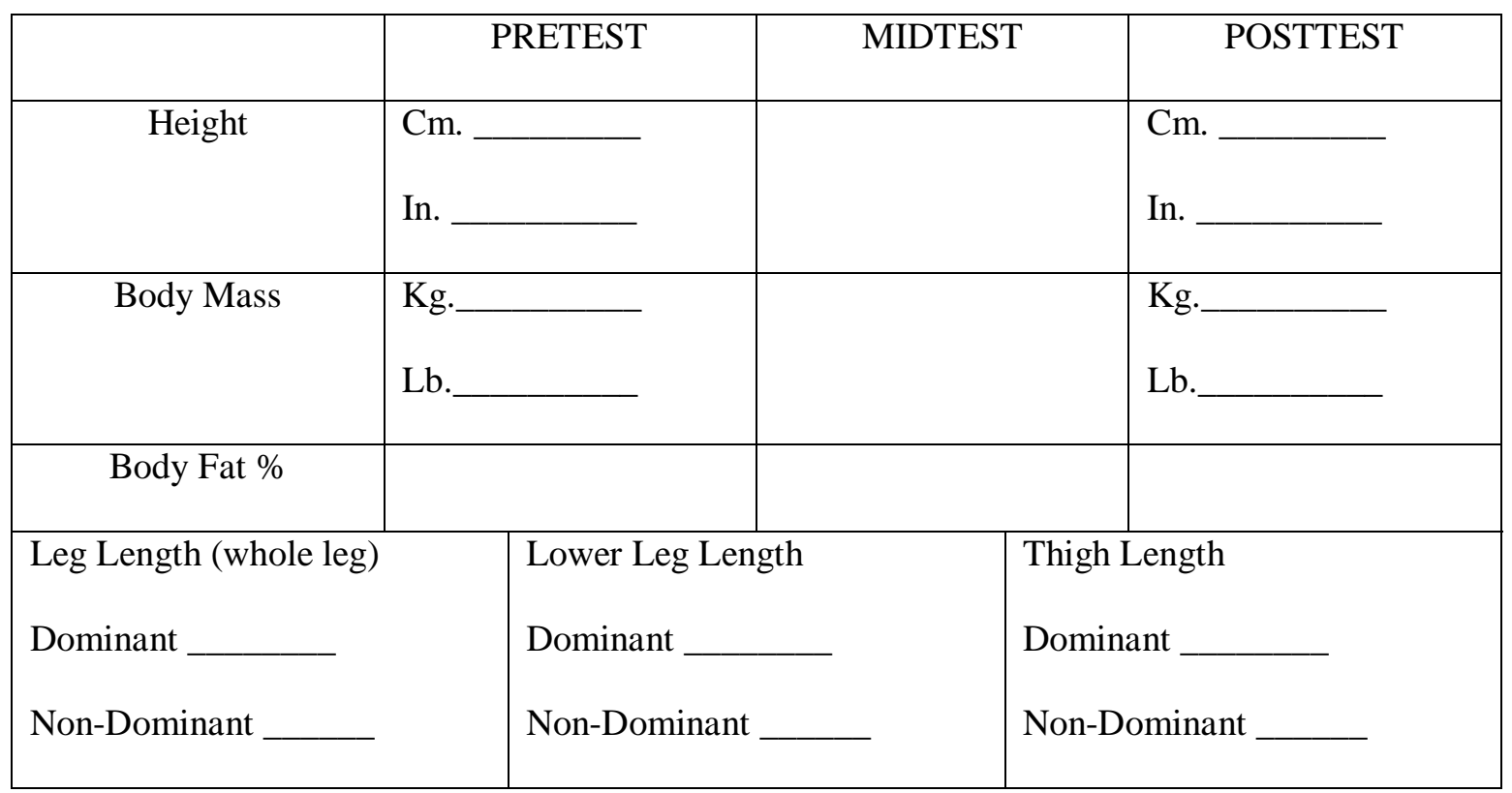

PERFORMANCE OUTCOME

\begin{tabular}{|c|c|c|c|}
\hline & PRETEST & MIDTEST & POSTTEST \\
\hline \multicolumn{4}{|l|}{ Power $(\mathrm{cm})$} \\
\hline \multicolumn{4}{|l|}{ Velcoity (sec) } \\
\hline $\begin{array}{l}\text { Peak Torque }(\mathrm{N} \cdot \mathrm{m}) \\
\text { Pre-Limb Wgt. = } \\
\text { Mid-Limb Wgt. = } \\
\text { Post-Limb Wgt. = }\end{array}$ & $\begin{array}{l}\text { Miometric-Peak Torque } \\
\text { Pliometric-Peak Torque }\end{array}$ & $\begin{array}{l}\text { Miometric-Peak Torque } \\
\text { Pliometric-Peak Torque }\end{array}$ & $\begin{array}{l}\text { Miometric-Peak Torque } \\
\text { Pliometric-Peak Torque }\end{array}$ \\
\hline Biodex Machine Settin & \multicolumn{3}{|c|}{$\begin{array}{ll}\text { 1. } \text { Power head height }= & \text { 3. Seat distance }= \\
\text { 2. } \text { Ankle height }= & \text { 4. Seat angle }= \\
\text { 3. } & \end{array}$} \\
\hline
\end{tabular}


Muscle Soreness/Pain Sensitivity

Rectus Femoris (Quadriceps), Biceps Femoris (Hamstrings), and Gastrocnemius (Calves)

\begin{tabular}{|c|c|c|c|}
\hline *FOR QUADS* & PRETEST & MIDTEST & POSTTEST \\
\hline Muscle Soreness & $\begin{array}{l}0 \mathrm{hr} \_ \\
48 \mathrm{hr} \\
96 \mathrm{hr}\end{array}$ & $\begin{array}{l}0 \mathrm{hr} \\
48 \mathrm{hr} \\
96 \mathrm{hr}\end{array}$ & $\begin{array}{l}0 \mathrm{hr} \\
48 \mathrm{hr} \\
96 \mathrm{hr} \\
\end{array}$ \\
\hline $\begin{array}{c}\text { Pain Sensitivity } \\
\text { DOMINANT LEG }\end{array}$ & $\begin{array}{l}0 \mathrm{hr} \\
48 \mathrm{hr} \\
96 \mathrm{hr} \\
\end{array}$ & $\begin{array}{l}0 \mathrm{hr} \\
48 \mathrm{hr} \\
96 \mathrm{hr}\end{array}$ & $\begin{array}{l}0 \mathrm{hr} \\
48 \mathrm{hr} \\
96 \mathrm{hr}\end{array}$ \\
\hline *FOR HAMS* & PRETEST & MIDTEST & POSTTEST \\
\hline Muscle Soreness & $\begin{array}{l}0 \mathrm{hr}- \\
48 \mathrm{hr} \\
96 \mathrm{hr}\end{array}$ & $\begin{array}{l}0 \mathrm{hr} \\
48 \mathrm{hr} \\
96 \mathrm{hr}\end{array}$ & $\begin{array}{l}0 \mathrm{hr} \\
48 \mathrm{hr} \\
96 \mathrm{hr}\end{array}$ \\
\hline $\begin{array}{c}\text { Pain Sensitivity } \\
\text { DOMINANT LEG }\end{array}$ & $\begin{array}{l}0 \mathrm{hr} \\
48 \mathrm{hr} \\
96 \mathrm{hr} \\
\end{array}$ & $\begin{array}{l}0 \mathrm{hr}- \\
48 \mathrm{hr}- \\
96 \mathrm{hr}\end{array}$ & $\begin{array}{l}0 \mathrm{hr}- \\
48 \mathrm{hr} \\
96 \mathrm{hr}\end{array}$ \\
\hline *FOR CALVES* & PRETEST & MIDTEST & POSTTEST \\
\hline Muscle Soreness & $\begin{array}{l}0 \mathrm{hr} \_ \\
48 \mathrm{hr} \\
96 \mathrm{hr}\end{array}$ & $\begin{array}{l}0 \mathrm{hr}- \\
48 \mathrm{hr} \\
96 \mathrm{hr}\end{array}$ & $\begin{array}{l}0 \mathrm{hr} \\
48 \mathrm{hr} \\
96 \mathrm{hr}\end{array}$ \\
\hline $\begin{array}{c}\text { Pain Sensitivity } \\
\text { DOMINANT LEG }\end{array}$ & $\begin{array}{l}0 \mathrm{hr} \\
48 \mathrm{hr} \\
96 \mathrm{hr}\end{array}$ & $\begin{array}{l}0 \mathrm{hr}- \\
48 \mathrm{hr}- \\
96 \mathrm{hr}\end{array}$ & $\begin{array}{l}0 \mathrm{hr} \\
48 \mathrm{hr} \\
96 \mathrm{hr}\end{array}$ \\
\hline
\end{tabular}

